\title{
On the cohomology of representations up to homotopy of Lie groupoids
}

\author{
Fernando Studzinski Carvalho
}

\author{
TESE APRESENTADA \\ AO \\ Instituto DE MATEMÁticA E EstatísticA \\ DA \\ Universidade de SÃo PAUlo \\ PARA \\ OBTENÇÃO DO TÍTULO \\ $\mathrm{DE}$ \\ DOUTOR EM CIÊNCIAS \\ Programa: Matemática \\ Orientador: Prof. Dr. Cristian Ortiz
}

Durante o desenvolvimento deste trabalho o autor recebeu auxílio financeiro da FAPESP e da CAPES, processo n²015/01698-7, Fundação de Amparo à Pesquisa do Estado de São

Paulo (FAPESP)

São Paulo, Novembro de 2019 


\title{
On the cohomology of representations up to homotopy of Lie groupoids
}

\begin{abstract}
Esta versão da tese contém as correções e alterações sugeridas pela Comissão Julgadora durante a defesa da versão original do trabalho, realizada em 25/11/2019. Uma cópia da versão original está disponível no Instituto de Matemática e Estatística da Universidade de São Paulo.
\end{abstract}

Comissão Julgadora:

- Prof. Dr. Cristian Andres Ortiz Gonzalez (orientador) - IME-USP

- Prof. Dr. Ivan Struchiner - IME-USP

- Prof. Dr. Marcos Benevenuto Jardim - UNICAMP

- Prof. Dr. Olivier Brahic - UFPR

- Prof. Dr. Matias Luis del Hoyo - UFF 


\section{Agradecimentos}

Gostaria de agradecer meu orientador Cristian Ortiz pelo suporte e amizade durante todos esses anos, principalmente pela sua compreensão do aspecto humano envolvido no decorrer de um doutorado. Tenho certeza que você infuenciou muito o modo como eu penso em matemática e na pesquisa, também sou grato por isso. Neste sentido, também gostaria de agradecer a todos os professores que de alguma forma contribuíram para a minha formação durante o doutorado, em especial meu agradecimento vai para os professores Matias del Hoyo e Ivan Struchiner, pelos seus valiosos ensinamentos e pelas discussões que tivemos nos últimos quatro anos.

Agradeço aos membros da comissão julgadora pelas correções e sugestões apresentadas.

Agradeço aos meus colegas do grupo(ide) de seminários em geometria de Poisson e tópicos relacionados: Camilo, Cristian, Genaro, Fernando, Jackeline e Pedro, pelas muitas conversas estimulantes sobre matemática.

Também agradeço a todos os meus amigos: Pedro, Luis, Adriana, Icaro, Carioca, Jackeline, Carol, Kelly, Oscar, Sidnei, Lorena, Marcelo e Felipe, pelos bons momentos que passamos juntos.

Agradeço a sorte que tive de poder participar da olímpiada de matemática das escolas públicas, ainda na sua primeira edição em 2005. Esse foi um grande marco na minha vida que abriu as portas da universidade para mim e me apresentou à ideia de pesquisa em matemática.

Agradeço imensamente a meus pais Laura e Renato, que embora não tenham tido a oportunidade de acesso à uma educação formal decente são pessoas com uma inteligência que admiro muito e que sempre me apoiaram incondicionalmente a continuar meus estudos. Espero poder continuar dando orgulho a vocês.

Agradeço à Mayumi, por estar sempre ao meu lado já há tantos anos, tenho certeza de que sou uma pessoa melhor graças a você. Obrigado pelo apoio emocional durante os momentos finais do doutorado e por compartilhar comigo essa vida simples e alegre.

Finalmente, agradeço à FAPESP e à CAPES pela bolsa de doutorado concedida através do processo $n^{\circ}$ 2015/01698-7, Fundação de Amparo à Pesquisa do Estado de São Paulo (FAPESP), sem a qual nada disso teria sido possível. 


\section{Resumo}

Studzinski, F. Sobre a cohomologia de representações a menos de homotopia de grupoides de Lie. Tese (Doutorado) - Instituto de Matemática e Estatística, Universidade de São Paulo, São Paulo, 2019.

Estudamos o conceito de representações a menos de homotopia de grupoides de Lie e a cohomologia naturalmente associada a tais representações. Nosso principal resultado é a prova de que a cohomologia de um grupoide de Lie com valores em uma representação a menos de homotopia é um invariante de Morita, o que pode ser interpretado como uma forma de introduzir invariantes cohomologicos para orbifolds e mais geralmente para stacks diferenciáveis, que são espaços com singularidades cujas classes de isomorfismo estão em correspondência biunívoca com classes de equivalência de Morita de grupoides de Lie. Para provar tal resultado, utilizamos a teoria de objetos simpliciais em categorias suaves e.g. variedades simpliciais, fibrados vetoriais simpliciais e equivalências entre eles, definidas a partir de mapas chamados hypercovers. Demonstramos também a invariância da cohomologia simplicial destes objetos sob hypercovers.

Palavras-chave: Grupoide de Lie, Representação a menos de homotopia, variedade simplicial, cohomologia. 


\section{Abstract}

Studzinski, F. On the cohomology of representations up to homotopy of Lie groupoids. Tese (Doutorado) - Instituto de Matemática e Estatística, Universidade de São Paulo, São Paulo, 2019.

We study the concept of representations up to homotopy of Lie groupoids. Our main result is the proof that the cohomology of a Lie groupoid with coefficients in a representation up to homotopy is a Morita invariant of the groupoid. This can be interpreted as a way to provide cohomological invariants for orbifolds and more generally for differentiable stacks, which are spaces with singularities whose isomorphism classes are in one-to-one correspondence with Morita equivalence classes of Lie groupoids. To prove this result, we rely on the theory of simplicial objects in smooth categories e.g. simplicial manifolds, simplicial vector bundles, and equivalences between them which are defined in terms of maps called hypercovers. We also prove results on the invariance of the simplicial cohomology of these spaces under hypercovers.

Keywords: Lie groupoid, representation up to homotopy, simplicial manifold, cohomology. 


\section{Contents}

$\begin{array}{lr}\text { Introduction } & 1\end{array}$

1 Lie groupoids and representations up to homotopy 5

1.1 Lie groupoids and Morita equivalences . . . . . . . . . . . . . . 5

1.2 The differentiable cohomology of a Lie groupoid . . . . . . . . . . . . . . . 10

1.3 Representations up to homotopy of a Lie groupoid . . . . . . . . . . . . . . 13

1.4 Two term representations up to homotopy . . . . . . . . . . . . . . . 19

1.4.1 The adjoint representation . . . . . . . . . . . . . 21

1.4 .2 VB-groupoids . . . . . . . . . . . . . . . . . 24

2 A brief introduction to simplicial objects 29

2.1 Simplicial sets . . . . . . . . . . . . . . . . . . . . . 29

2.2 Simplicial spaces . . . . . . . . . . . . . . . . . . . 31

2.3 Hypercovers and equivalences between simplicial manifolds . . . . . . . . . . . . . . . . . . . . 36

2.4 The de Rham cohomology of a simplicial manifold . . . . . . . . . . . . . 42

$3 \quad$ The category of simplicial vector bundles $\quad 48$

3.1 A study of linear hypercovers . . . . . . . . . . . . . . 50

3.2 Hypercover invariance of the linear cohomology . . . . . . . . . . . . 51

3.3 From representations up to homotopy to simplicial vector bundles . . . . . 53

4 Morita invariance of the cohomology with values in a representation up to homotopy $\quad 56$

4.1 The complex of projectable cochains . . . . . . . . . . . . . 56

4.2 Morita invariance of the linear cohomology . . . . . . . . . . . . 60

4.3 Morita invariance of the cohomology with values in a representation up to homotopy ....................... 62

$\begin{array}{lr}\text { Bibliography } & 68\end{array}$ 


\section{Introduction}

Representation theory is a very broad and rich area of research in mathematics. One can study representations of almost any algebraic structure, for instance, representations of groups, associative algebras, Lie algebras, etc. A Lie group $G$ is usually the group of symmetries of an abstract space e.g. a smooth manifold, it can also act as the symmetries of a physical system as in e.g. a hamiltonian action, and the study of representations of $G$ is a way to better understand the structure of the group by viewing it as symmetries of a simpler space, usually a finite-dimensional vector space.

A groupoid $\mathcal{G}$ consists of a set of objects $\mathcal{G}_{0}$ and a set of arrows (morphisms) $\mathcal{G}_{1}$ together with structural maps:

- $s$ called source and $t$ called target; where $s, t: \mathcal{G}_{1} \rightarrow \mathcal{G}_{0}$ are given by $s(y \stackrel{g}{\leftarrow} x)=x$ and $t(y \stackrel{g}{\leftarrow} x)=y$;

- a partially defined multiplication $m: \mathcal{G}_{1}{ }_{s} \times{ }_{t} \mathcal{G}_{1} \rightarrow \mathcal{G}_{1}$ given by $m(h, g)=h g$, where $\mathcal{G}_{1}{ }_{s} \times{ }_{t} \mathcal{G}_{1}=\left\{((h, g)) \in \mathcal{G}_{1} \times \mathcal{G}_{1} \mid s(h)=t(g)\right\}$ is the set of composable arrows, satisfying the associative property $m(g h, k)=(g h) k=g(h k)=m(g, h k)$ whenever the compositions are defined;

- a unit map $1: \mathcal{G}_{0} \rightarrow \mathcal{G}_{1}$ given by $1(x)=1_{x}$, satisfying for all arrows $(y \stackrel{g}{\leftarrow} x) \in \mathcal{G}_{1}$ the identities $g 1_{x}=g$ and $1_{y} g=g$;

- an inversion map $i: \mathcal{G}_{1} \rightarrow \mathcal{G}_{1}$ given by $i(y \stackrel{g}{\leftarrow} x)=x \stackrel{g^{-1}}{\longleftarrow} y$, that for every arrow $g \in \mathcal{G}_{1}$ satisfies the identities $g g^{-1}=1_{y}$ and $g^{-1} g=1_{x}$.

Lie groupoids were introduced by C. Ehresmann in the late 1950's to describe smooth symmetries of a smooth family of objects. That is, in a Lie groupoid both the set of arrows $\mathcal{G}_{1}$ and the set of objects $\mathcal{G}_{0}$ are manifolds, source and target maps are submersions and multiplication, unit and inversion structural maps are smooth. Thus, a Lie groupoid $\mathcal{G}_{1} \rightrightarrows \mathcal{G}_{0}$ can be thought of as a smooth collection of symmetries parametrized by the points in the base manifold $\mathcal{G}_{0}$. Hence, it is natural to think of representations of the Lie groupoid as a way to view $\mathcal{G}$ as symmetries between fibres of a vector bundle $E$ over $\mathcal{G}_{0}$. This turns out to be a well-defined notion of representation but with a clear downside: while a Lie group $G$ always admits a representation on a vector space $V$, and more importantly always 
have canonical representations, like the adjoint and coadjoint representations on its Lie algebra, for Lie groupoids, this fails to happen and in general, a representation of $\mathcal{G}$ on $E$ may impose strong restrictions on $E$. Moreover, $\mathcal{G}$ may have no representation on its Lie algebroid.

This is one of the reasons why M. Crainic and C. Arias Abad [3] introduced the notion of representations up to homotopy of a Lie groupoid. The idea is that instead of asking $\mathcal{G}$ to act on a vector bundle $E$ one should allow a graded vector bundle $E_{\bullet}$ and a sequence of operators: a differential $\partial$ turning $\left(E_{\bullet}, \partial\right)$ into a chain complex, a chain map $\lambda^{g}:\left(\left.E_{\bullet}\right|_{x}, \partial\right) \rightarrow\left(\left.E_{\bullet}\right|_{y}, \partial\right)$ for each arrow $y \stackrel{g}{\leftarrow} x$, a chain homotopy $\gamma^{g, h}$ between $\lambda^{g h}$ and $\lambda^{g} \lambda^{h}$ for each pair of composable arrows $(g, h)$, together with higher-order operators and higher coherence equations. This way, the groupoid $\mathcal{G}$ acts on a complex of vector bundles $\left(E_{\bullet}, \partial\right)$ by chain maps and the usual associative property is asked to hold only up to homotopy of complexes. Using this definition, they were able to provide adjoint and coadjoint representations for any Lie groupoid and extend many results of the theory of representations of Lie groups to the context of Lie groupoids. For instance, the cohomology of the classifying space $B \mathcal{G}$, which is computed via the Bott-Shulman-Stasheff double complex, can be better understood looking at the differentiable cohomology of $\mathcal{G}$ with values in a symmetric power of the coadjoint representation up to homotopy of $\mathcal{G}$ (see [3]).

Lie groupoids are interesting for many reasons, for instance they provide a unified framework to deal with problems on smooth manifolds, actions of Lie groups on manifolds, principal bundles etc; they also appear in connection to foliation theory [29], problems in mathematical physics, e.g. symplectic groupoids are the integration of integrable Poisson manifolds [48], and noncommutative geometry [13,32]. Moreover, they can also serve as smooth models for spaces with singularities such as orbifolds [44, 33], or more generally differentiable stacks $[8,6,7,50]$. Any Lie groupoid $\mathcal{G}_{1} \rightrightarrows \mathcal{G}_{0}$ has associated to it a topological space $\mathcal{G}_{1} / \mathcal{G}_{0}$, called the orbit space of the Lie groupoid, obtained from an orbit equivalence relation on the base $\mathcal{G}_{0}$, where two points are in the same orbit if there is an arrow $g \in \mathcal{G}_{1}$ between them. Particular important examples are when $\mathcal{G}$ is the action groupoid of a Lie group action on a manifold, where its orbit space is exactly the orbit space of the action; and when $\mathcal{G}$ is the holonomy groupoid of a foliation, in which case the orbit space is the leaf space of the foliation. An orbifold is a topological space which is locally modelled as the quotient space of an open set in $\mathbb{R}^{n}$ by a finite group action. Thus, it is a generalization of a smooth manifold where we think of points with non-trivial isotropy group as singularities. In the aforementioned correspondence, what happens is that any orbifold can be realized as the orbit space of some proper and étale Lie groupoid, and isomorphic orbifolds are represented in this way by Morita equivalent groupoids.

The Morita equivalence relation between Lie groupoids is the best suited for many applications. It is the one capable of capturing the geometric information transverse to the 
orbits of the groupoid, for instance, Morita equivalent groupoids have homeomorphic orbit spaces and isomorphic normal representations [22]. As for stacks, they are objects originally studied in algebraic geometry, but recently there has been an increasing interest in what is called a differentiable stack. These stacks can be thought as smooth structures describing generalized quotients of smooth manifolds and it is well known that isomorphism classes of differentiable stacks are in correspondence with Morita equivalence classes of Lie groupoids, where a Lie groupoid is seen as a presentation (or an atlas) for a differentiable stack. Therefore, it is usual that in order to do concrete calculations and constructions on stacks one passes to a presentation. In this thesis, we do not explore the technical theory of stacks and simply think of them as Morita equivalence classes of Lie groupoids.

Taking this point of view, it is natural to think of providing Morita invariants of Lie groupoids as a natural way to provide invariants for the spaces with singularities represented by them. In this thesis, we are going to show that the cohomology with coefficients in a representation up to homotopy is one such Morita invariant, thus yielding a cohomology invariant for orbifolds, and more generally for differentiable stacks. In order to do that we will rely on the theory of simplicial manifolds and simplicial vector bundles. In the last decade, A. Henriques [30], J. P. Pridham [45], E. Getzler [25], C. Zhu [50], among others, have developed the theory of Lie $\infty$-groupoids, that is: simplicial manifolds that satisfy a smooth analogue of the so-called Kan extension conditions (or horn-filling conditions) from simplicial homotopy theory. These higher groupoids include, for each $n \geqslant 0$, the class of Lie $n$-groupoids, which are $\infty$-groupoids such that the horn-fillings are unique above dimension $n$. Also recently, see for instance $[35,41,40]$, it has been proposed that symplectic Lie 2-groupoids are the global objects integrating certain Courant algebroids, this show us that higher groupoids are also important and natural when one studies symplectic or Poisson geometry and their related fields. Examples of this higher groupoids include: Lie 0-groupoids, that are precisely smooth manifolds, and Lie 1-groupoids, that are precisely nerves of Lie groupoids. Since representations up to homotopy appeared, many results have been proved about them, in particular in [28] it has been shown that representations up to homotopy of $\mathcal{G}$ on two-term complexes are in one-to-one correspondence with VB-groupoids over $\mathcal{G}$, where a VB-groupoid is a groupoid object in the category of vector bundles, moreover, this correspondence was later shown to be an equivalence of categories [19]. The main idea is that for more general representations up to homotopy, a similar result holds, but the geometric object corresponding to such a representation is a higher Lie groupoid which is somehow a vector bundle over $\mathcal{G}$. This correspondence is the subject of a work in progress by M. del Hoyo and G. Trentinaglia [21] and is made precise using the notion of simplicial vector bundles over the nerve of a Lie groupoid. In this thesis, we study the concepts of simplicial manifolds and simplicial vector bundles. We make use of the natural simplicial cohomologies associated to such objects in order to obtain a simpler description of the co- 
homology of a Lie groupoid with values in a representation up to homotopy, and settle the Morita invariance of this cohomology.

The contents of the thesis are as follows:

In Chapter One, we briefly review the literature on Lie groupoids and representations up to homotopy, providing the main definitions, examples and setting the notations and conventions that will be used throughout the text. We also review the notion of VB-groupoids and their close relationship with representations up to homotopy on two-term complexes.

In Chapter Two, we introduce simplicial objects, focusing on simplicial manifolds and equivalences between them, which are defined in terms of special maps called hypercovers. In the last part of the chapter, we show that the differentiable cohomology of a simplicial manifold is an invariant under hypercovers using some results of [26] on the de Rham cohomology of simplicial manifolds.

In Chapter Three, we study the category of simplicial vector bundles, since these are the abstract objects generalizing VB-groupoids that will later be shown to be related to representations up to homotopy. We define the linear complex $\left(C_{\operatorname{lin}}^{\bullet}(V)\right)$, associated to a simplicial vector bundle $V$, considering differentiable cochains that are linear on the fibres of $V$. We also define the notion of linear hypercover between simplicial vector bundles, simply considering hypercovers that take into account the linear structure, and we show that the cohomology of the linear complex is invariant under linear hypercovers.

In Chapter Four, we explain the construction provided by del Hoyo and Trentinaglia for the semidirect product of a Lie groupoid $\mathcal{G}$ with a representation up to homotopy. This construction gives rise to a simplicial vector bundle over the nerve of the groupoid $\mathcal{G}$. Their work generalizes at the same time the semidirect product construction for 2-term representations and the Dold-Kan correspondence.

Chapter Five contains the main contributions of this thesis. We study the linear cohomology of simplicial vector bundles coming from representations up to homotopy. We define a complex of projectable cochains which generalizes the VB-groupoid complex defined in [28] and we show that its cohomology computes the cohomology of the dual of the representation up to homotopy we started with. We also show that the inclusion of the complex of projectable cochains into the linear complex is a quasi-isomorphism and that the cohomology of both is a Morita invariant, therefore settling the Morita invariance of the cohomology with values in a representation up to homotopy. 


\section{Chapter 1}

\section{Lie groupoids and representations up to homotopy}

In this chapter, we briefly review the literature on Lie groupoids and representations up to homotopy, providing the main definitions, examples and setting the notations and conventions that will be used throughout the text. We also review the notion of VB-groupoids and their close relationship with representations up to homotopy on two-term complexes. All of this is standard and can be found elsewhere. For the general theory on Lie groupoids we follow mainly $[43,38]$, and for the theory on representations up to homotopy and VBgroupoids we follow $[3,19,28,12]$.

\subsection{Lie groupoids and Morita equivalences}

In this section, we introduce Lie groupoids and morphisms between them. We also define the notion of Morita equivalence between Lie groupoids and provide some examples.

A groupoid $\mathcal{G}$ is a small category where all morphisms are isomorphisms. Concretely, this can rephrased by saying that $\mathcal{G}$ consists of a set of objects $\mathcal{G}_{0}$ and a set of arrows (morphisms) $\mathcal{G}_{1}$ together with structural maps:

- $s$ called source and $t$ called target; where $s, t: \mathcal{G}_{1} \rightarrow \mathcal{G}_{0}$ are given by $s(y \stackrel{g}{\leftarrow} x)=x$, and $t(y \stackrel{g}{\leftarrow} x)=y$;

- a partially defined multiplication $m: \mathcal{G}_{1}{ }_{s} \times{ }_{t} \mathcal{G}_{1} \rightarrow \mathcal{G}_{1}$ given by $m(h, g)=h g$, where $\mathcal{G}_{1}{ }_{s}{ }_{t} \mathcal{G}_{1}=\left\{((h, g)) \in \mathcal{G}_{1} \times \mathcal{G}_{1} \mid s(h)=t(g)\right\}$ is the set of composable arrows, satisfying the associative property $m(g h, k)=(g h) k=g(h k)=m(g, h k)$ whenever the compositions are defined;

- a unit map $1: \mathcal{G}_{0} \rightarrow \mathcal{G}_{1}$ given by $1(x)=1_{x}$, satisfying for all arrows $(y \stackrel{g}{\leftarrow} x) \in \mathcal{G}_{1}$ the identities $g 1_{x}=g$ and $1_{y} g=g$; 
- an inversion map $i: \mathcal{G}_{1} \rightarrow \mathcal{G}_{1}$ given by $i(y \stackrel{g}{\leftarrow} x)=x \stackrel{g^{-1}}{\longleftarrow} y$, that for every arrow $g \in \mathcal{G}_{1}$ satisfies the identities $g g^{-1}=1_{y}$ and $g^{-1} g=1_{x}$.

Definition 1.1.1. A Lie groupoid is a groupoid $\mathcal{G}$, such that the spaces of objects and arrows $\mathcal{G}_{0}$ and $\mathcal{G}_{1}$ are both smooth manifolds, where the source and target maps $s$ and $t$ are surjective submersions and all the other structure maps $m, 1, i$ are smooth.

Remark 1.1.2. All the manifolds considered by us will be assumed to be Hausdorff and second countable topological spaces.

Example 1.1.3. Any Lie group $G$ can be seen as a Lie groupoid where the manifold of objects consists of only one point. This way, $G \rightrightarrows\{*\}$ is a Lie groupoid where the source and target are trivial projections, the unit map $1:\{*\} \rightarrow G$ gives the unit element of the group, and the multiplication and inversion maps are the multiplication and inversion of the group.

Example 1.1.4. If $M$ is a smooth manifold then we have the unit groupoid $M \rightrightarrows M$, where every structure map is the identity map.

Example 1.1.5. If $M$ is a manifold, the pair groupoid of $M$ is given by $M \times M \rightrightarrows M$, where $s(y, x)=x, t(y, x)=y, m((z, y),(y, x))=(z, x), i(y, x)=(x, y)$ and $1(x)=(x, x)$.

Example 1.1.6. Suppose that $G$ is a Lie group acting on a smooth manifold $M$ on the left. Then, we can form what is called the action groupoid $G \times M \rightrightarrows M$. The source map is the projection $s(g, x)=x$, and the target is given by the action $t(g, x)=g x$. The unit is $1(x)=(1, x)$, the multiplication is $m((g, x),(h, y))=(g h, y)(x$ must coincide with hy in order that $m$ is defined), and the inversion is $i(g, x)=\left(g^{-1}, g x\right)$.

Example 1.1.7. Let $M$ be a smooth manifold, the fundamental groupoid of $M$ is the Lie groupoid $\Pi(M) \rightrightarrows M$, such that an arrow with source $x$ and target $y$ is the homotopy class with fixed endpoints of a path $\gamma:[0,1] \rightarrow M$, satisfying $\gamma(0)=x$ and $\gamma(1)=y$. The multiplication is given by concatenation of paths, a unit arrow is a constant path, and the inversion of a homotopy class of a path is the class of the path traversed in the opposite direction.

Example 1.1.8. Suppose $p: M \rightarrow N$ is a surjective submersion. Then, we can consider the following Lie groupoid $M_{p} \times{ }_{p} M \rightrightarrows M$, with structural maps:

$$
\begin{aligned}
& \text { - } s, t: M_{p} \times_{p} M \rightarrow M ; s(y, x)=x \text { e } t(y, x)=y . \\
& \text { - } m((z, y),(y, x))=(z, x) . \\
& \text { - } 1: M \rightarrow M_{p} \times_{p} M ; 1(x)=(x, x) . \\
& \text { - } i: M_{p} \times_{p} M \rightarrow M_{p} \times_{p} M ; i(y, x)=(x, y) .
\end{aligned}
$$

This Lie groupoid is called the submersion groupoid associated to $p$. 
Example 1.1.9. Consider $M$ a smooth manifold and $\left\{\mathcal{U}_{\alpha}\right\}_{\alpha \in \Lambda}$ an open cover of $M$. Then, we have a canonical submersion $p: \bigsqcup_{\alpha} \mathcal{U}_{\alpha} \rightarrow M$, such that $\left.p\right|_{\mathcal{U}_{\alpha}}$ is the inclusion of $\mathcal{U}_{\alpha}$ inside $M$. In this case, the submersion groupoid associated to $p$ is of the form $\bigsqcup_{\alpha, \beta} \mathcal{U}_{\alpha} \cap \mathcal{U}_{\beta} \rightrightarrows M$. The Lie groupoid obtained in this way is called the Čech groupoid of the open cover.

Example 1.1.10. Let $E \rightarrow M$ be a smooth vector bundle. Then, we can define a Lie groupoid $\mathrm{GL}(E) \rightrightarrows M$, where an arrow $(y \stackrel{g}{\leftarrow} x) \in \mathrm{GL}(E)$ is, by definition, a linear isomorphism from the fibre $\left.E\right|_{x}$ to $\left.E\right|_{y}$. This Lie groupoid is called the general linear groupoid of $E$. It is a generalisation of the general linear group $\operatorname{GL}(V)$ associated to a vector space $V$.

Definition 1.1.11. If $\mathcal{G}_{1} \rightrightarrows \mathcal{G}_{0}$ is a Lie groupoid, then for each $x \in \mathcal{G}_{0}$ we define the isotropy group of $x$ as the space of all arrows with source and target equal to $x$

$$
\mathcal{G}_{x}:=\left\{g \in \mathcal{G}_{1} ; \text { such that } s(g)=x=t(g)\right\}
$$

We define the orbit of $x$ as

$$
\mathcal{O}_{x}=\left\{y \in \mathcal{G}_{0} \mid \text { there is } g \in \mathcal{G}_{1} \text {, such that } s(g)=x, t(g)=y\right\} .
$$

And since the orbits give a partition of $\mathcal{G}_{0}$ by equivalence classes, we define the orbit space as $\mathcal{G}_{0} / \mathcal{G}_{1}$, the quotient space of $\mathcal{G}_{0}$ by this orbit relation.

This notion of orbit space of a Lie groupoid is very important, for instance in the case of an action groupoid (Example 1.1.6), the orbit space of the groupoid is the usual quotient space of the action. This is just one of the reasons why the study of Lie groupoids can be very important and helpful because we can study properties and invariants of possibly complicated quotient spaces using the Lie groupoid as a smooth model for that space.

The following proposition gives a good description of the geometric information encoded in the structure of a Lie groupoid. It is standard in the the literature, see [43, 38].

Proposition 1.1.12. Let $\mathcal{G}_{1} \rightrightarrows \mathcal{G}_{0}$ be a Lie groupoid, and $x, y \in \mathcal{G}_{0}$. Then, the following holds:

- the set $\mathcal{G}(y, x)$, of all arrows from $x$ to $y$, is an embedded submanifold of $\mathcal{G}_{1}$;

- the isotropy group $\mathcal{G}_{x}$ is a Lie group;

- the orbit $\mathcal{O}_{x}$ has the structure of an immersed submanifold of $\mathcal{G}_{0}$;

- the $s$-fibre, $s^{-1}(x)$, is a $\mathcal{G}_{x}$-principal bundle over $\mathcal{O}_{x}$, with projection $t: s^{-1}(x) \rightarrow \mathcal{O}_{x}$;

- denoting by $\mathcal{N}_{x} \mathcal{O}:=T_{x} \mathcal{G}_{0} / T_{x} \mathcal{O}$, the fibre of the normal bundle to the orbit inside $\mathcal{G}_{0}$, we have a natural representation of $\mathcal{G}_{x}$ on $\mathcal{N}_{x} \mathcal{O}$, defined byg $\cdot v=d t(X)$, for any $X \in T_{g} \mathcal{G}_{1}$ such that $d s(X)=v$. This representation is called the normal representation of $\mathcal{G}_{x}$. 
Definition 1.1.13. Let $\mathcal{G}_{1} \rightrightarrows \mathcal{G}_{0}$ be a Lie groupoid. We say that $\mathcal{G}$ is a proper Lie groupoid if the map $(t, s): \mathcal{G}_{1} \rightarrow \mathcal{G}_{0} \times \mathcal{G}_{0}$ is a proper map i.e. the inverse image of a compact is also compact. We also say that $\mathcal{G}$ is étale if the isotropy groups $\mathcal{G}_{x}$ are discrete for every $x \in \mathcal{G}_{0}$.

Proper Lie groupoids are a generalisation of the concept of proper actions of Lie groups. This follows from the fact that an action groupoid (Example 1.1.6) is a proper Lie groupoid if, and only if, the action is proper. One can show that in a proper Lie groupoid $\mathcal{G}$, the isotropy groups $\mathcal{G}_{x}$ are compact Lie groups, the orbits $\mathcal{O}_{x}$ are embedded submanifolds of $\mathcal{G}_{0}$, and the orbit space $\mathcal{G}_{0} / \mathcal{G}_{1}$ is Hausdorff, among many other nice properties, including a Linearisation Theorem, which generalizes the usual Slice/Tube Theorem for proper actions of Lie groups (as in [23]). For more details on proper Lie groupoids see [18, 22, 16].

In particular, orbit spaces of proper Lie groupoids have more structure, in the literature they are usually referred to as orbispaces $[22,47,16]$. A particular and very important special instance is given by orbit spaces of a proper and étale groupoids since, in this case, the isotropy groups must be finite and thus, the orbit space has a natural structure of an orbifold [44].

Since groupoids are small categories, it is natural to define morphisms between them simply as functors. When we are concerned with Lie groupoids, the morphisms will also be functors, but we have to account for the smooth structures involved:

Definition 1.1.14. Consider $\mathcal{G}_{1} \rightrightarrows \mathcal{G}_{0}$ and $\mathcal{H}_{1} \rightrightarrows \mathcal{H}_{0}$ two Lie groupoids, then a Lie groupoid morphism from $\mathcal{G}$ to $\mathcal{H}$ consists of a pair of smooth maps $\phi_{1}: \mathcal{G}_{1} \rightarrow \mathcal{H}_{1}$ and $\phi_{0}: \mathcal{G}_{0} \rightarrow \mathcal{H}_{0}$ commuting with all the structure maps of $\mathcal{G}$ and $\mathcal{H}$. Concretely, for every $g \in \mathcal{G}_{1}, x \in \mathcal{G}_{0}$ and $\left(g_{2}, g_{1}\right) \in \mathcal{G}_{s} \times{ }_{t} \mathcal{G}$, they must satisfy

- $\phi_{0}(s(g))=s\left(\phi_{1}(g)\right)$ and $\phi_{0}(t(g))=t\left(\phi_{1}(g)\right)$;

- $\phi_{1}\left(1_{x}\right)=1_{\left(\phi_{0}(x)\right)}$;

- $\phi_{1}\left(m\left(g_{2}, g_{1}\right)\right)=m\left(\phi_{1}\left(g_{2}\right), \phi_{1}\left(g_{1}\right)\right)$.

where we have used the same notation for the structure maps of $\mathcal{G}$ and $\mathcal{H}$.

Let us recall now the definition of Morita equivalence between Lie groupoids.

Definition 1.1.15. A map of Lie groupoids $\phi: \mathcal{G} \rightarrow \mathcal{H}$ is called Morita if it satisfies the following two conditions:

1. The map $\mathcal{H}_{1}{ }_{s} \times_{\phi_{0}} \mathcal{G}_{0} \rightarrow \mathcal{H}_{0}$, given by $(g, x) \mapsto t(g)$ is a surjective submersion.

2. The following square is a pullback of manifolds

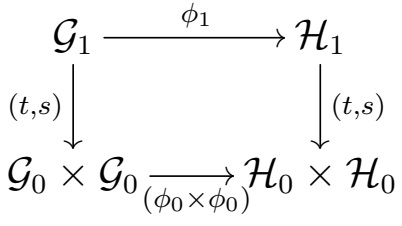


Note that, in the terminology of category theory, forgetting about the smooth structure, condition 1 is saying that $\phi$ is essentially surjective and condition 2 is equivalent to $\phi$ being full and faithful.

It is a standard fact that any morphism of Lie groupoids $\phi: \mathcal{G} \rightarrow \mathcal{H}$ takes orbits to orbits and induces a continuous map, $\phi_{*}: \mathcal{G}_{0} / \mathcal{G}_{1} \rightarrow \mathcal{H}_{0} / \mathcal{H}_{1}$, between orbit spaces. It also yields maps between isotropy groups $\phi_{x}: \mathcal{G}_{x} \rightarrow \mathcal{H}_{\phi_{0}(x)}$, and between normal spaces $d_{x} \phi: \mathcal{N}_{x} \mathcal{O}_{x} \rightarrow \mathcal{N}_{\phi_{0}(x)} \mathcal{O}_{\phi_{0}(x)}$, which put together give a morphism between normal representations.

The following theorem borrowed from [22] gives a complete characterisation of Morita maps in terms of these maps induced between orbit spaces and normal representations.

Theorem 1.1.16. A morphism of Lie groupoids $\phi: \mathcal{G} \rightarrow \mathcal{H}$ is a Morita map if, and only if, it induces a homeomorphism $\phi_{*}: \mathcal{G}_{0} / \mathcal{G}_{1} \rightarrow \mathcal{H}_{0} / \mathcal{H}_{1}$ between orbit spaces, and isomorphisms between normal representations $\phi_{x}:\left(\mathcal{G}_{x} \curvearrowright \mathcal{N}_{x} \mathcal{O}_{x}\right) \rightarrow\left(\mathcal{H}_{\phi_{0}(x)} \curvearrowright \mathcal{N}_{\phi_{0}(x)} \mathcal{O}_{\phi_{0}(x)}\right)$.

Definition 1.1.17. A morphism of Lie groupoids $\phi: \mathcal{G} \rightarrow \mathcal{H}$ is called a Morita fibration if it is a Morita map, such that the map $\phi_{0}: \mathcal{G}_{0} \rightarrow \mathcal{H}_{0}$ is a surjective submersion.

Definition 1.1.18. We say that two Lie groupoids $\mathcal{G}$ and $\mathcal{H}$ are Morita equivalent if there are a third Lie groupoid $\mathcal{K}$ and Morita fibrations $\phi: \mathcal{K} \rightarrow \mathcal{G}$ and $\psi: \mathcal{K} \rightarrow \mathcal{H}$.

Remark 1.1.19. Usually when defining Morita equivalences one does that using Morita maps (which are only essentially surjective on objects). Here we are using Morita fibrations which are surjective submersions on objects. This causes no conflict because if there are a Lie groupoid $\mathcal{K}$ and Morita maps $\phi: \mathcal{K} \rightarrow \mathcal{G}$ and $\psi: \mathcal{K} \rightarrow \mathcal{H}$ there always exist a new Lie groupoid $\tilde{\mathcal{K}}$ and Morita fibrations $\tilde{\phi}: \tilde{\mathcal{K}} \rightarrow \mathcal{G}$ and $\tilde{\psi}: \tilde{\mathcal{K}} \rightarrow \mathcal{H}$.

Example 1.1.20. Consider $G \rightrightarrows\{*\}$ and $H \rightrightarrows\{*\}$, two Lie groups viewed as Lie groupoids over a point. Suppose that they are Morita equivalent, then there exists $\mathcal{K}$ and Morita maps: $\phi: \mathcal{K} \rightarrow G$ and $\psi: \mathcal{K} \rightarrow H$. Since Morita maps induce isomorphisms between isotropy groups, it follows that $\phi$ and $\psi$ induce isomorphisms $\mathcal{K}_{z} \rightarrow H_{y}=H$ and $\mathcal{K}_{z} \rightarrow G_{x}=G$. Thus, $G$ and $H$ are Morita equivalent if, and only if, they are isomorphic Lie groups.

Example 1.1.21. Let $M_{p} \times{ }_{p} M \rightrightarrows M$ be the submersion groupoid associated to $p: M \rightarrow N$. Then, the morphism of Lie groupoids

$$
\phi:\left(M_{p} \times{ }_{p} M \rightrightarrows M\right) \rightarrow(N \rightrightarrows N)
$$

given by $\phi_{1}(y, x)=p(x)$, and $\phi_{0}(x)=p(x)$ is a Morita fibration of Lie groupoids, between the submersion groupoid and the unit groupoid of $N$. Therefore, they are Morita equivalent. In particular, the Čech groupoid $\bigsqcup_{\alpha, \beta} \mathcal{U}_{\alpha} \cap \mathcal{U}_{\beta} \rightrightarrows N$ from an open cover of a smooth manifold $N$ is Morita equivalent to $N \rightrightarrows N$. 
Example 1.1.22. If $G$ is a Lie group with a free and proper action on a manifold $M$. Then, there is a unique smooth structure on the quotient space $M / G$, such that the canonical projection $p: M \rightarrow M / G$ is a surjective submersion. Thus, we can consider the action Lie groupoid $G \ltimes M \rightrightarrows M$ and the submersion groupoid $M_{p} \times{ }_{p} M \rightrightarrows M$. Note that, the map $G \ltimes M \rightarrow M_{p} \times{ }_{p} M$, given by $(g, x) \mapsto(x, g x)$ is an isomorphism of Lie groupoids. By the previous example, we know that $M_{p} \times_{p} M$ is Morita equivalent to $M / G \rightrightarrows M / G$. Therefore, $G \ltimes M$ is also Morita equivalent to the unit groupoid $M / G \rightrightarrows M / G$.

The previous example, although very simple, can be used as a motivation. In a way, it explains how Morita equivalent Lie groupoids, in this case the action groupoid $G \ltimes M$ and the submersion groupoid $M / G \rightrightarrows M / G$, serve as smooth models for the same quotient space $M / G$. For instance, we could start with a proper action of $G$ on $M$ which only locally free, meaning that all its isotropy groups are finite. Then, the quotient $M / G$ is no longer a manifold in general. It will be an orbifold, and any Lie groupoid in the Morita equivalence class of $G \ltimes M \rightrightarrows M$ will represent the same orbifold structure on the quotient $M / G$, (up to isomorphism).

\subsection{The differentiable cohomology of a Lie groupoid}

The differentiable cohomology of a Lie groupoid is a natural cohomology associated to any Lie groupoid, it generalizes the usual cohomology of Lie groups, and comes from the natural simplicial structure of the nerve of the Lie groupoid (which will appear in chapter 2). In what follows, we will use the spaces of composable arrows of lengths $k \in \mathbb{N}$, defined by $\mathcal{G}_{k}=\left\{\left(g_{k}, \ldots, g_{1}\right) \mid s\left(g_{i+1}\right)=t\left(g_{i}\right), \forall i \in\{1, \ldots, k-1\}\right\}$.

Definition 1.2.1. For any Lie groupoid $\mathcal{G}_{1} \rightrightarrows \mathcal{G}_{0}$, we have an associated cochain complex defined in the following way: $C^{0}(\mathcal{G})=C^{\infty}\left(\mathcal{G}_{0}, \mathbb{R}\right)$, for each $p \geqslant 1, C^{p}(\mathcal{G})=C^{\infty}\left(\mathcal{G}_{p}, \mathbb{R}\right)$, with a differential $\delta: C^{p}(\mathcal{G}) \rightarrow C^{p+1}(\mathcal{G})$ given by

$$
\begin{aligned}
\delta(f)\left(g_{p+1}, \ldots, g_{1}\right) & =f\left(g_{p}, \ldots, g_{1}\right) \\
& +\sum_{i=1}^{p}(-1)^{p+1-i} f\left(g_{p+1}, \ldots, g_{i+1} g_{i}, \ldots, g_{1}\right) \\
& +(-1)^{p+1} f\left(g_{p+1}, \ldots, g_{2}\right) .
\end{aligned}
$$

for $p \geqslant 1$, and $\delta: C^{0}(\mathcal{G}) \rightarrow C^{1}(\mathcal{G})$, defined by $\delta(f)\left(g_{1}\right)=(f \circ s)\left(g_{1}\right)-(f \circ t)\left(g_{1}\right)$.

The cohomology associated to this complex is denoted $H_{\text {diff }}^{\bullet}(\mathcal{G})$ and is called the differentiable cohomology of $\mathcal{G}$.

Remark 1.2.2. We remark that, the pair $\left(C^{\bullet}(\mathcal{G}), \delta\right)$ can be made into a differential graded algebra for the product $C^{p}(\mathcal{G}) \times C^{q}(\mathcal{G}) \rightarrow C^{p+q}(\mathcal{G}) ;\left(f_{1}, f_{2}\right) \mapsto f_{1} * f_{2}$, defined by: 
- $f_{1} * f_{2}\left(g_{p}, \ldots, g_{1}\right)=f_{1}\left(g_{p}, \ldots, g_{1}\right) f_{2}\left(s\left(g_{1}\right)\right) \quad$ if $q=0$;

- $f_{1} * f_{2}\left(g_{p+q}, \ldots, g_{1}\right)=f_{1}\left(g_{p+q}, \ldots, g_{q+1}\right) f_{2}\left(g_{q}, \ldots g_{1}\right) \quad$ if $p, q \neq 0$

- $f_{1} * f_{2}\left(g_{p+q}, \ldots, g_{1}\right)=f_{1}\left(t\left(g_{q}\right)\right) f_{2}\left(g_{q}, \ldots g_{1}\right) \quad$ if $p=0$;

and if both $p$ and $q$ are zero $f_{1} * f_{2}=f_{1} f_{2}$. That is, $\delta$ acts as a derivation for this product:

$$
\delta\left(f_{1} * f_{2}\right)=\delta\left(f_{1}\right) * f_{2}+(-1)^{p} f_{1} \delta\left(f_{2}\right)
$$

for any $\left(f_{1}, f_{2}\right) \in C^{p}(\mathcal{G}) \times C^{q}(\mathcal{G})$.

Definition 1.2.3. Let $\mathcal{G}_{1} \rightrightarrows \mathcal{G}_{0}$ be a Lie groupoid. Then, a representation of $\mathcal{G}$ is a vector bundle $E$ over the base $\mathcal{G}_{0}$, together with a morphism of Lie groupoids $\Delta: \mathcal{G}_{1} \rightarrow \operatorname{GL}(E)$ to the general linear groupoid (see Example 1.1.10), that is the identity on $\mathcal{G}_{0}$. Thus, in a representation each arrow $g \in G L(E)$ with source $x$ and target $y$ gives rise to a linear isomorphism $\Delta_{g}:\left.\left.E\right|_{x} \rightarrow E\right|_{y}$.

Example 1.2.4. It is easy to see that if the Lie groupoid $\mathcal{G}$ is actually a Lie group $G$, then this notion of representation recovers the usual notion of representation of a Lie group, since in this case $\mathcal{G}_{0}$ is a point, a vector bundle $E$ over $\mathcal{G}_{0}$ is just a vector space, and $G$ acts on $E$ in a linear manner.

Example 1.2.5. If $M$ is a smooth manifold and $E$ is a vector bundle over $M$, then $E$ has the structure of a representation of the pair groupoid of $M$ if and only if $E$ is a trivial vector bundle.

Example 1.2.6. Let $M$ be a smooth manifold and $\Pi(M)$ be its fundamental groupoid. Then,a vector bundle $E$ over $M$ has the structure of a representation of $\Pi(M)$ if and only if $E$ has a flat connection.

The last two examples show that this notion of representation of a Lie groupoid is very restrictive, so that some Lie groupoids may have very few representations.

Now if $\mathcal{G}$ has a representation on a vector bundle $E$ over the base $\mathcal{G}_{0}$, we also have a notion of differentiable cohomology with coefficients in the representation $\mathrm{E}$.

Definition 1.2.7. For each $g=\left(g_{k}, \ldots, g_{1}\right) \in \mathcal{G}_{k}$ we denote by $\pi_{j}: \mathcal{G}_{k} \rightarrow \mathcal{G}_{0}$ the projection to the $j$-th object, that is $\pi_{j}(g)=t\left(g_{j}\right)$ for all $j \geq 1$ and $\pi_{0}(g)=s\left(g_{1}\right)$. Let $\mathcal{G}_{1} \rightrightarrows \mathcal{G}_{0}$ be a Lie groupoid and $E \rightarrow \mathcal{G}_{0}$ be a vector bundle which is a representation of $\mathcal{G}$ via the map $\Delta^{E}: \mathcal{G}_{1} \rightarrow G L(E)$. Then, we define the complex $\left(C^{\bullet}(\mathcal{G}, E), D\right)$, where for each $k \geqslant 1$, the space of $k$-cochains is the space of sections $\Gamma\left(\pi_{k}^{*} E, \mathcal{G}_{k}\right)$ of the vector bundle $\pi_{k}^{*} E$ over $\mathcal{G}_{k}$. Thus, a cochain $c$ can be seen as a map $c: \mathcal{G}_{k} \rightarrow E,\left.\left(g_{k}, \ldots, g_{1}\right) \mapsto c\left(g_{k}, \ldots, g_{1}\right) \in E\right|_{t\left(g_{k}\right)}$, 
and we have a differential $D: C^{k}(\mathcal{G}, E) \rightarrow C^{k+1}(\mathcal{G}, E)$ given by

$$
\begin{aligned}
D(c)\left(g_{k+1}, \ldots, g_{1}\right) & =\Delta_{g_{k+1}}^{E}\left(c\left(g_{k}, \ldots, g_{1}\right)\right) \\
& +\sum_{i=1}^{k}(-1)^{k+1-i} c\left(g_{k+1}, \ldots, g_{i+1} g_{i}, \ldots, g_{1}\right) \\
& +(-1)^{k+1} c\left(g_{k+1}, \ldots, g_{2}\right) .
\end{aligned}
$$

For $k=0$, we define $C^{0}(\mathcal{G}, E):=\Gamma\left(E, \mathcal{G}_{0}\right)$ and $D: \Gamma\left(E, \mathcal{G}_{0}\right) \rightarrow C^{1}(\mathcal{G}, E)$ is given by $D(c)\left(g_{1}\right)=\Delta_{g_{1}}^{E}\left(c\left(s\left(g_{1}\right)\right)\right)-c\left(t\left(g_{1}\right)\right)$.

The cohomology associated to this complex is denoted $H_{\text {diff }}^{\bullet}(\mathcal{G}, E)$ and is called the differentiable cohomology of $\mathcal{G}$ with values in the representation $E$.

Remark 1.2.8. In the case of a trivial representation of a Lie groupoid $\mathcal{G}_{1} \rightrightarrows \mathcal{G}_{0}$ on the trivial vector bundle $E=\mathcal{G}_{0} \times \mathbb{R}$, the differentiable cohomology with values in $E$ recovers the differentiable cohomology $H_{\text {diff }}^{\bullet}(\mathcal{G})$ of the groupoid $\mathcal{G}$.

Example 1.2.9. If we consider a Lie group $G$ with Lie algebra $\mathfrak{g}$ and its adjoint representation $A d: G \rightarrow G L(\mathfrak{g})$, the complex associated is given by: $C^{0}(G, \mathrm{Ad})=\mathfrak{g}$, and $C^{k}(G, \mathrm{Ad})=$ $C^{\infty}(\underbrace{G \times \ldots \times G}_{k \text { times }}, \mathfrak{g})$ for $k \geqslant 1$. For $k \geqslant 1$ the differential is

$$
\begin{aligned}
\delta(c)\left(g_{k+1}, \ldots, g_{1}\right) & =\operatorname{Ad}_{g_{k+1}}\left(c\left(g_{k}, \ldots, g_{1}\right)\right. \\
& +\sum_{i=1}^{k}(-1)^{k+1-i} c\left(g_{k+1}, \ldots, g_{i+1} g_{i}, \ldots, g_{1}\right) \\
& +(-1)^{k+1} c\left(g_{k+1}, \ldots, g_{1}\right) .
\end{aligned}
$$

and for $k=0, \delta_{0}: C^{0}(\mathcal{G}, \mathrm{Ad}) \rightarrow C^{1}(\mathcal{G}, \mathrm{Ad})$ is given by

$$
\begin{aligned}
\delta_{0}: \mathfrak{g} & \rightarrow C^{\infty}(G, \mathfrak{g}) \\
v & \mapsto \delta(v)(g)=\operatorname{Ad}_{g}(v)-v
\end{aligned}
$$

This cohomology with coefficients in the adjoint representation is usually important for various reasons, for instance one of them is that it is isomorphic to the deformation cohomology of the Lie group $G$, so it controls deformations of the Lie group [17].

Proposition 1.2.10. Suppose that $\mathcal{G}_{1} \rightrightarrows \mathcal{G}_{0}$ is a Lie groupoid with a representation on a vector bundle $E \rightarrow \mathcal{G}_{0}$, then, the complex $\left(C^{\bullet}(\mathcal{G}, E), D\right)$ has a natural structure of differential graded right $C^{\bullet}(\mathcal{G})$-module defined by

$$
\begin{aligned}
C^{p}(\mathcal{G}, E) \times C^{q}(\mathcal{G}) & \rightarrow C^{p+q}(\mathcal{G}, E) \\
(c, f) & \mapsto(c * f)\left(g_{p+q}, \ldots, g_{1}\right)=c\left(g_{p+q}, \ldots, g_{q+1}\right) f\left(g_{q}, \ldots, g_{1}\right)
\end{aligned}
$$


That is, the degree one operator $D$ satisfies $D^{2}=0$ and the Leibniz identity

$$
D(c * f)=D(c) * f+(-1)^{p} c * \delta(f)
$$

for any $c$ of degree $p$.

Definition 1.2.11. A quasi-action of a Lie groupoid $\mathcal{G}_{1} \rightrightarrows \mathcal{G}_{0}$ on a vector bundle $E \rightarrow \mathcal{G}_{0}$ is a map $\Delta^{E}: \mathcal{G} \rightarrow \operatorname{End}(E)$ that associates for each arrow $y \stackrel{g}{\leftarrow} x$ a linear map $\Delta_{g}:\left.E\right|_{x} \rightarrow$ $\left.E\right|_{y}$. It is said to be unital if $\Delta_{1_{x}}$ is the identity of the fibre $\left.E\right|_{x}$.

Remark 1.2.12. The spaces $C^{k}(\mathcal{G}, E)=\Gamma\left(\pi_{k}^{*} E, \mathcal{G}_{k}\right)$ make sense for any vector bundle $E \rightarrow$ $\mathcal{G}_{0}$. Also, if there is $\Delta^{E}: \mathcal{G} \rightarrow \operatorname{End}(E)$ a quasi-action, there is an induced operator $\delta_{\Delta}:$ $C^{\bullet}(\mathcal{G}, E) \rightarrow C^{\bullet}(\mathcal{G}, E)$ defined by the same formula of the $\delta$ above (see Definition 1.2.7). But a quasi-action will be an action if and only if it is unital and satisfies $\Delta_{g_{2} g_{1}}=\Delta_{g_{2}} \circ \Delta_{g_{1}}$, for every pair of composable arrows $\left(g_{2}, g_{1}\right)$. The latter condition turns out to be equivalent to $\delta_{\Delta}^{2}=0$, so in general we do not get a cochain complex from a quasi-action.

Proposition 1.2.13. There is a one-to-one correspondence between quasi-actions of a Lie groupoid $\mathcal{G}$ on a vector bundle $E$ and degree one linear operators

$$
D: C^{\bullet}(\mathcal{G}, E) \rightarrow C^{\bullet}(\mathcal{G}, E)
$$

satisfying the Leibniz identity with respect to the $C^{\bullet}(\mathcal{G})$-module structure. Explicitly, we can recover the quasi-action $\lambda$ from $D$, by looking at degree zero elements $c \in \Gamma(E)$ and defining:

$$
\lambda(g)(c(s(g)))=c(t(g))+D(c)(g) .
$$

A quasi action $\lambda$ is in fact a representation, if and only if, $\lambda\left(1_{x}\right)(v)=v$, and the corresponding operator D squares to zero.

A fundamental result in [14] shows that the cohomology of a groupoid with coefficients in a representation is a Morita invariant, i.e. if $\phi: \mathcal{G} \rightarrow \mathcal{H}$ is a Morita map between two groupoids and $E$ is a representation of $\mathcal{H}$, then for each $k \in \mathbb{N}$ we have:

$$
H_{\text {diff }}^{k}(\mathcal{H}, E) \cong H_{\text {diff }}^{k}\left(\mathcal{G}, \phi^{*} E\right),
$$

where $\phi^{*} E$ is a representation of $\mathcal{G}$ given by the pullback of $E$ by $\phi: \mathcal{G} \rightarrow \mathcal{H}$. In particular, the cohomology $H_{\text {diff }}^{\bullet}(\mathcal{G}, E)$ is an invariant for the stack presented by the groupoid $\mathcal{G}$.

\subsection{Representations up to homotopy of a Lie groupoid}

As we mentioned before, representations of a Lie groupoid as defined in the previous section are too strict for many practical reasons. In this section, we introduce the notion of 
representations up to homotopy of a Lie groupoid, as defined by Crainic and Arias Abad in [3]. The main idea is to substitute vector bundles for graded vector bundles and to consider more general differential graded modules over $C^{\bullet}(\mathcal{G})$ than the ones coming from usual representations obtained in Proposition 1.2.10.

Let $\mathcal{G}_{1} \rightrightarrows \mathcal{G}_{0}$ be a Lie groupoid, and consider a graded vector bundle over the base $\mathcal{G}_{0}$, that is $\mathcal{E}=\bigoplus_{j \geqslant 0} E_{j}$, where each $E_{j}$ is a vector bundle over $\mathcal{G}_{0}$. Then, we can define a space of $\mathcal{E}$-valued cochains $C(\mathcal{G}, \mathcal{E})^{\bullet}=\oplus_{p} C(\mathcal{G}, \mathcal{E})^{p}$ by

$$
C(\mathcal{G}, \mathcal{E})^{p}=\bigoplus_{k-j=p} C^{k}\left(\mathcal{G}, E_{j}\right)
$$

where as before $C^{k}\left(\mathcal{G}, E_{j}\right)=\Gamma\left(\pi_{k}^{*} E_{j}, \mathcal{G}_{k}\right)$.

Given $c \in C^{k}\left(\mathcal{G}, E_{l}\right)$, we say that it has bidegree $(k, l)$, where $\langle c\rangle=k$ is called its cocycle degree, and $|c|=k-l$ is called its total degree.

We can also decompose $C(\mathcal{G}, \mathcal{E})^{\bullet}$ with respect to the cocycle degree, we put $C^{p}(\mathcal{G}, \mathcal{E})=$ $\oplus_{i \geqslant 0} C^{p}\left(\mathcal{G}, \mathcal{E}_{i}\right)$ and

$$
C(\mathcal{G}, \mathcal{E})^{\bullet}=\oplus_{p} C^{p}(\mathcal{G}, \mathcal{E})
$$

After all these definitions, it is easy to see that $C(\mathcal{G}, E)^{\bullet}$ has a right $C^{\bullet}(\mathcal{G})$-module structure, defined in the same way as in Proposition 1.2.10. So, we can now extend our notion of representation of a Lie groupoid to the graded setting.

Definition 1.3.1. Let $\mathcal{G}$ be a Lie groupoid and $\mathcal{E}=\oplus_{j=0}^{l} E_{j}$ be a graded vector bundle over $\mathcal{G}_{0}$. Then, a representation up to homotopy of $\mathcal{G}$ on $\mathcal{E}$ is a structure of differential graded right $C^{\bullet}(\mathcal{G})$-module on $C(\mathcal{G}, \mathcal{E})^{\bullet}$. In other words, it is a linear degree one operator

$$
D: C(\mathcal{G}, E)^{\bullet} \rightarrow C(\mathcal{G}, E)^{\bullet}
$$

satisfying $D^{2}=0$, and the Leibniz rule with respect to the $C^{\bullet}(\mathcal{G})$-module structure:

$$
D(c * f)=D(c) * f+(-1)^{|c|} c * \delta(f)
$$

The cohomology associated to this complex is $H_{\text {diff }}^{\bullet}(\mathcal{G}, \mathcal{E})$, defined by:

$$
H_{\text {diff }}^{p}(\mathcal{G}, \mathcal{E}):=\frac{\operatorname{ker}\left(D: C(\mathcal{G}, \mathcal{E})^{p} \rightarrow C(\mathcal{G}, \mathcal{E})^{p+1}\right)}{\operatorname{Im}\left(D: C(\mathcal{G}, \mathcal{E})^{p-1} \rightarrow C(\mathcal{G}, \mathcal{E})^{p}\right)}
$$

for each $p \geqslant 0$, and is its called the differentiable cohomology of $\mathcal{G}$ with coefficients in the representation up to homotopy $\mathcal{E}$.

Definition 1.3.2. A morphism $\Phi: \mathcal{E} \rightarrow \mathcal{E}^{\prime}$ between two representations up to homotopy of $\mathcal{G}$ is a degree zero map

$$
\Phi: C(\mathcal{G}, \mathcal{E})^{\bullet} \rightarrow C\left(\mathcal{G}, \mathcal{E}^{\prime}\right)^{\bullet}
$$


that commutes with the differentials of $\mathcal{E}$ and $\mathcal{E}^{\prime}$

With these definitions, we a have a category $\operatorname{Rep}(\mathcal{G})$ of representations up to homotopy of a Lie groupoid $\mathcal{G}$, which is nothing more than a subcategory of the category of differential graded modules over the differential graded algebra $C^{\bullet}(\mathcal{G})$.

This is the compact version of the definition, in what follows we will see a more explicit version that will make clear why this is called a representation up to homotopy.

One can see that $C(\mathcal{G}, \mathcal{E})^{\bullet}$ as a module over $C^{\bullet}(\mathcal{G})$ is the same as $\Gamma(\mathcal{E}) \otimes_{C^{\infty}\left(\mathcal{G}_{0}\right)} C^{\bullet}(\mathcal{G})$, where the tensor structure over $C^{\infty}\left(\mathcal{G}_{0}\right)$ satisfies $\eta f_{1} \otimes f_{2}=\eta \otimes f_{1} * f_{2}$, for every $\eta \in \Gamma(\mathcal{E})$, every $f_{1} \in C^{\infty}\left(\mathcal{G}_{0}\right)$ and every $f_{2} \in C^{\bullet}(\mathcal{G})$. Thus, $C(\mathcal{G}, \mathcal{E})^{\bullet}$ is generated by $\Gamma(\mathcal{E})$ as a right $C^{\bullet}(\mathcal{G})$-module.

This fact, plus the fact that the operator $D$ is a derivation for the module structure, implies that $D$ is determined by its action on the space of sections $\Gamma(\mathcal{E})$. By decomposing $D$ with respect to the cocycle grading we can write it as:

$$
D=D_{0}+D_{1}+D_{2}+\ldots
$$

where each $D_{i}$ raises the cocycle degree by i (and the total degree by one), so that

$$
D_{i}: C^{k}\left(\mathcal{G}, E_{j}\right) \rightarrow C^{k+i}\left(\mathcal{G}, E_{j+i-1}\right) .
$$

We know that these are the only possibilities for the $D_{i}$ also because $D$ is a derivation, so it can not lower the cocycle degree. Hence, one obtains the following decomposition for the operator $D$ defining a representation up to homotopy, as explained in [3]:

Proposition 1.3.3. Any representation up to homotopy of a Lie groupoid $\mathcal{G}$ on a graded vector bundle $\mathcal{E}=\oplus E_{j}$ given by an operator $D$ decomposes as

$$
D=D_{0}+D_{1}+D_{2}+\ldots
$$

where each $D_{i}$ raises the cocycle degree by $i$,

$$
D_{i}: C^{k}\left(\mathcal{G}, E_{l}\right) \rightarrow C^{k+i}\left(\mathcal{G}, E_{l+i-1}\right),
$$

for every $i \neq 1$, it satisfies

$$
D_{i}(c * f)=D_{i}(c) * f,
$$

for $i=1$, it satisfies

$$
D_{1}(c * f)=D_{1}(c) * f+(-1)^{|c|} c * \delta(f),
$$

and for each $k \geqslant 0$, the operators fit in the equation:

$$
D_{0} D_{k}+D_{1} D_{k-1}+\ldots D_{k} D_{0}=0 \text {. }
$$


The following proposition, also from [3], gives a characterization for the operators $D_{i}$ above.

Proposition 1.3.4. There is a one-to-one correspondence between:

- maps $\Phi: C^{k}\left(\mathcal{G}, E_{l}\right) \rightarrow C^{k+i}\left(\mathcal{G}, E_{l+j}\right)$, such that $\phi(c * f)=\Phi(c) * f$.

- cochains $\Phi^{\prime} \in C^{i}\left(\mathcal{G}, \operatorname{End}_{j}\left(E_{\bullet}\right)\right):=\Gamma\left(\mathcal{G}_{i+j} ; \operatorname{Hom}^{(i+j-1)}\left(\pi_{0}^{*} E_{\bullet}, \pi_{i+j}^{*} E_{\bullet}\right)\right.$.

The correspondence is as follows:

$$
\Phi(c)\left(g_{k+i}, \ldots, g_{1}\right)=(-1)^{(|c|+<c>)} \Phi^{\prime}\left(g_{k+i}, \ldots, g_{k+1}\right) c\left(g_{k}, \ldots, g_{1}\right) .
$$

The definition of representation up to homotopy given in Definition 1.3.1 is nice because it is concise and recovers ordinary representations in the context of differential graded modules over $C^{\bullet}(\mathcal{G})$. But to understand this definition a little bit better it is very useful to have the decomposition given in Proposition 1.3.3, and the correspondence given by Proposition 1.3.4. With these results put together, we can give a new characterization of representations up to homotopy.

Given $\mathcal{G}_{1} \rightrightarrows \mathcal{G}_{0}$ a Lie groupoid, we consider its nerve $\left(\mathcal{G}_{m}\right)_{m \geqslant 0}$, where for $m \geqslant 2$ an element $g \in \mathcal{G}_{m}$ is a sequence of composable arrows

$$
g=\left(x_{m} \stackrel{g_{m}}{\longleftarrow} x_{m-1} \cdots x_{2} \stackrel{g_{2}}{\longleftarrow} x_{1} \stackrel{g_{1}}{\longleftarrow} x_{0}\right),
$$

with face maps $d_{1}, d_{0}: \mathcal{G}_{1} \rightarrow \mathcal{G}_{0}$, defined by $d_{1}=s$, and $d_{0}=t$, and more generally $d_{i}: \mathcal{G}_{n} \rightarrow \mathcal{G}_{n-1}$, for $i \in\{0, \ldots, n\}$ defined by:

$$
\left\{\begin{array}{l}
d_{0}\left(g_{n}, g_{n-1}, \ldots, g_{1}\right)=\left(g_{n}, \ldots, g_{2}\right) \\
d_{j}\left(g_{n}, g_{n-1}, \ldots, g_{n}\right)=\left(g_{1}, \ldots, g_{j+1} g_{j}, \ldots, g_{1}\right) \quad 1 \leq j \leq n-1 \\
d_{n}\left(g_{n}, g_{n-1}, \ldots, g_{1}\right)=\left(g_{n-1}, \ldots, g_{1}\right)
\end{array}\right.
$$

Moreover, we denote by $s_{k}: \mathcal{G}_{m} \rightarrow \mathcal{G}_{k}$ and $t_{m-k}: \mathcal{G}_{m} \rightarrow \mathcal{G}_{m-k}$, the first $k$-face and the last $(m-k)$-face of a $g \in \mathcal{G}_{m}$. Thus, $s_{k}(g)$ and $t_{m-k}(g)$ are given by:

$$
s_{k}(g)=\left(x_{k} \stackrel{g_{k}}{\longleftarrow} x_{k-1} \cdots x_{1} \stackrel{g_{1}}{\longleftarrow} x_{0}\right), \quad t_{m-k}(g)=\left(x_{m} \stackrel{g_{m}}{\longleftarrow} \cdots x_{k+1} \stackrel{g_{k+1}}{\longleftarrow} x_{k}\right)
$$

Then, in view of the previous discussion, decomposing the structure operator $D$ of a representation up to homotopy in terms of the cocycle degree, and applying Proposition 1.3.4, for each of the operators $D_{m}$ in Proposition 1.3.3, we obtain that a representation up to homotopy of $\mathcal{G}$ on a graded vector bundle $\mathcal{E}=\oplus E_{j}$ over $\mathcal{G}_{0}$ is equivalent to a sequence of operators:

$$
R_{m} \in \Gamma\left(\mathcal{G}_{m} ; \operatorname{Hom}^{(m-1)}\left(\pi_{0}^{*} E_{\bullet}, \pi_{m}^{*} E_{\bullet}\right)\right) \quad m \geq 0,
$$


satisfying the following equations, for every $g \in \mathcal{G}_{m}$, and $m \geq 0$ :

$$
\sum_{k=1}^{m-1}(-1)^{k} R_{m-1}^{d_{k}(g)}=\sum_{k=0}^{m}(-1)^{k} R_{m-k}^{t_{m-k}(g)} \circ R_{k}^{s_{k}(g)}
$$

For low values of $m$ they amount to the following:

$$
\begin{aligned}
R_{0}^{x_{0}} \circ R_{0}^{x_{0}} & =0 & & x_{0} \in \mathcal{G}_{0} \\
R_{0}^{x_{1}} R_{1}^{g_{1}} & =R_{1}^{g_{1}} R_{0}^{x_{0}} & & g_{1} \in \mathcal{G}_{1} \\
R_{1}^{g_{2}} R_{1}^{g_{1}}-R_{1}^{g_{2} g_{1}} & =R_{2}^{g_{2}, g_{1}} R_{0}^{x_{0}}+R_{0}^{x_{2}} R_{2}^{g_{2}, g_{1}} & & \left(g_{2}, g_{1}\right) \in \mathcal{G}_{2}
\end{aligned}
$$

In other words, these equations can be interpreted as follows:

- For each $x_{0} \in \mathcal{G}_{0}$, the map $R_{0}^{x_{0}}$ is a differential on the fiber $E_{\bullet}^{x_{0}}$ of $E_{\bullet}$ over $x_{0}$, turning $\left(E_{\bullet}, R_{0}\right)$ into a chain complex.

- For each arrow $x_{1} \stackrel{g_{1}}{\longleftarrow} x_{0}$, we have a quasi-action $R_{1}^{g_{1}}: E_{\bullet}^{x_{0}} \rightarrow E_{\bullet}^{x_{1}}$, that is a chain map.

- For each pair of composable arrows $\left(x_{2} \stackrel{g_{2}}{\longleftarrow} x_{1} \stackrel{g_{1}}{\longleftarrow} x_{0}\right) \in \mathcal{G}_{2}$, the map $R_{2}^{g_{2}, g_{1}}: E_{\bullet}^{x_{0}} \rightarrow$ $E_{\bullet+1}^{x_{2}}$ is a chain homotopy between $R_{1}^{g_{2}} \circ R_{1}^{g_{1}}$ and $R_{1}^{g_{2} g_{1}}$.

This justifies the name of representation up to homotopy. We relax the notion of representation of a Lie groupoid allowing it to act on chain complexes of vector bundles via chain maps, and the usual associativity condition for an action is satisfied only up to homotopies of chain complexes. Of course, we still have all other operators for $m$ bigger than two and higher-order coherences these operators must obey.

Example 1.3.5 (Usual Representations). Any representation of a Lie groupoid $\mathcal{G}_{1} \rightrightarrows \mathcal{G}_{0}$ on a vector bundle $E$ is an example of a representation up to homotopy concentrated in degree zero. In this case, every structure operator $R_{i}$, for $i \geqslant 2$, vanishes.

Example 1.3.6 (Cocycles). Any cocycle $\eta \in C^{k}(\mathcal{G})$ with $k \geqslant 2$ induces a representation up to homotopy in the complex which has the trivial line bundle $\mathbb{R}$ in degrees 0 and $k-1$ with differential equals to zero. The non-zero structure operators are given by $R_{1}=\lambda$ and $R_{k}=\eta$, where $\lambda$ denotes the trivial representation of $\mathcal{G}$ in $\mathbb{R}$.

Definition 1.3.7. A representation up to homotopy is called weakly unital if $R_{1}(g)=$ Id, whenever $g$ is a unit arrow. It is also called unital if, moreover, $R_{k}\left(g_{k}, \ldots, g_{1}\right)=0$ whenever $k \geqslant 2$, and any one of the arrows $g_{i}$ 's is a unit.

Now we will review some basic operations on representations up to homotopy, like taking pull-backs, dual representations and the cone of a morphism. There are also definitions 
of tensor products and symmetric powers. Their construction is somewhat complicated and thoroughly explained in [1]. Here we will give just a brief idea.

Example 1.3.8. For any morphism of Lie groupoids $\phi: \mathcal{G} \rightarrow \mathcal{H}$ there is a pullback functor naturally defined:

$$
\phi^{*}: \operatorname{Rep}(\mathcal{H}) \rightarrow \operatorname{Rep}(\mathcal{G})
$$

that at the level of objects takes any representation up to homotopy $\mathcal{E}=\left(E_{\bullet}, R_{0}, R_{1}, \ldots\right)$ of $\mathcal{H}$ to the representation $\phi^{*}(\mathcal{E})=\left(\phi^{*}\left(E_{\bullet}\right), \phi^{*}\left(R_{0}\right), \phi^{*}\left(R_{1}\right), \ldots\right)$ where the structure maps are defined by

$$
\phi^{*}\left(R_{k}\right)\left(g_{k}, \ldots, g_{1}\right):=R_{k}\left(\phi\left(g_{k}\right), \ldots, \phi\left(g_{1}\right)\right) .
$$

It is well-known that the category of ordinary representations of a groupoid is a Morita invariant, in the sense that Morita equivalent groupoids have equivalent categories of representations. Such an equivalence can be obtained using the pullback functor induced by a Morita map.

Other important constructions of representations up to homotopy are given in the next two examples.

Example 1.3.9. Let $\mathcal{G}$ be a Lie groupoid with a representation up to homotopy given by $\mathcal{E}=\left(E_{\bullet}, R_{0}, R_{1}, \ldots\right)$. Then its dual is the representation $\mathcal{E}^{*}=\left(E_{\bullet}^{*}, R_{0}^{*}, R_{1}^{*}, \ldots\right)$ of $\mathcal{G}$, where the structure operators are

$$
R_{k}^{*}\left(g_{k}, \ldots, g_{1}\right)=(-1)^{k+1}\left(R_{k}\left(g_{1}^{-1}, \ldots, g_{k}^{-1}\right)\right)^{*}
$$

Note that, with this convention and the notation $R_{0}=\partial$ for the differential, the chain complex structure on $E_{\bullet}^{*}$ has as differential $R_{0}^{*}=-\partial^{*}$, and that is not the standard dual of the chain complex $\left(E_{\bullet}, \partial\right)$, which usually has differential $(-1)^{n} \partial^{*}$ on $E_{n}^{*}$.

Example 1.3.10. We can also define mapping cones of morphisms between representations up to homotopy. First, recall that if $\phi_{0}:\left(E_{\bullet}, \partial_{E}\right) \rightarrow\left(F_{\bullet}, \partial_{F}\right)$ is a morphism of chain complexes of vector bundles, then the mapping cone is a new complex of vector bundles, denoted $C\left(\phi_{0}\right)$, defined by

$$
\left.C\left(\phi_{0}\right)_{n}=E_{n} \oplus F_{n+1}, \quad \partial(e, f)=\left(\partial_{E}(e)\right), \phi(e)-\partial_{F}(f)\right) .
$$

Now, if we are given a morphism between representations $\Phi: \mathcal{E} \rightarrow \mathcal{F}$ in $\operatorname{Rep}(\mathcal{G})$, we can construct a mapping cone $\mathcal{C}(\Phi) \in \operatorname{Rep}(\mathcal{G})$, whose underlying complex is the mapping cone of $\Phi_{0}$, and the structure operators are defined as

$$
R_{k}\left(g_{k}, \ldots, g_{1}\right)(e, f)=\left(R_{k}\left(g_{k}, \ldots, g_{1}\right) e, \phi_{k}\left(g_{k}, \ldots, g_{1}\right) e-(-1)^{k} R_{k}\left(g_{k}, \ldots, g_{1}\right) f\right)
$$

Viewed a differential graded module over $C^{\bullet}(\mathcal{G})$, this mapping cone $C(\Phi)$ is just the 
mapping cone complex of the morphism $\Phi: C(\mathcal{G}, \mathcal{E})^{\bullet} \rightarrow C(\mathcal{G}, \mathcal{F})^{\bullet}$.

Example 1.3.11. Given $\mathcal{E}$ and $\mathcal{E}^{\prime}$ representations up to homotopy of $\mathcal{G}$, one can define their tensor product $\mathcal{E} \otimes \mathcal{E}^{\prime}$. If $\left(R_{k}\right)_{k \geqslant 0}$ and $\left(R_{k}^{\prime}\right)_{k \geqslant 0}$ denote the structure operators of $\mathcal{E}$ and $\mathcal{E}^{\prime}$ respectively, then it is easy to define the structure operators of $\mathcal{E} \otimes \mathcal{E}^{\prime}$ in low degrees:

- The degree zero element is $R_{0} \otimes \mathrm{Id}+\mathrm{Id} \otimes R_{0}^{\prime}$, so that the underlying complex is the tensor product of the underlying complexes of $\mathcal{E}$ and $\mathcal{E}^{\prime}$.

- The degree one operator is the quasi-action given by the diagonal action.

As for the higher operators the definition is more subtle and depends on some choices. In the end, the tensor product representation will depend on these choices but different choices give rise to isomorphic representations up to homotopy, for more details see [1].

\subsection{Two term representations up to homotopy}

In what follows we will make more explicit the structure of a representation up to homotopy concentrated in degrees 0 and 1, i.e. in the case where the graded vector bundle is of the form $E^{0} \oplus E^{1}$. After that, we will show the main example of a two term representation, that is the adjoint representation up to homotopy of a Lie groupoid $\mathcal{G}_{1} \rightrightarrows \mathcal{G}_{0}$ on the graded vector bundle $\mathrm{Ad}:=T \mathcal{G}_{0} \oplus A \mathcal{G}$, where $A \mathcal{G}$ is the Lie algebroid of $\mathcal{G}$. In this section we follow closely the articles [28] and [19]. All the details and proofs can be found on these references.

Suppose that $\mathcal{E}=E_{0} \oplus E_{1}$ is a two term representation up to homotopy of a Lie groupoid $\mathcal{G}_{1} \rightrightarrows \mathcal{G}_{0}$. Then, by definition, for every $k \geqslant 0$ the space of $k$-cochains is given by

$$
C(\mathcal{G}, \mathcal{E})^{k}=C^{k}\left(\mathcal{G}, E_{0}\right) \oplus C^{k+1}\left(\mathcal{G}, E_{1}\right)
$$

and we have a degree 1 operator

$$
D: C^{k-1}\left(\mathcal{G}, E_{0}\right) \oplus C^{k}\left(\mathcal{G}, E_{1}\right) \rightarrow C^{k}\left(\mathcal{G}, E_{0}\right) \oplus C^{k+1}\left(\mathcal{G}, E_{1}\right)
$$

which written in components is necessarily of the form

$$
D\left(c_{0}, c_{1}\right)=\left(D^{E_{0}}\left(c_{0}\right)+\partial\left(c_{1}\right), D^{E_{1}}\left(c_{1}\right)+\Omega c_{0}\right)
$$

where:

- $\partial: E_{1} \rightarrow E_{0}$ is a vector bundle morphism and we use the same letter to designate the map induced in sections

$$
\partial: C^{k}(\mathcal{G}, C) \rightarrow C^{k}(\mathcal{G}, E), c \mapsto \partial(c)=\partial \circ c
$$


- $D^{E_{0}}, D^{E_{1}}$ are operators that raise the cocycle degree by one, and correspond to quasiactions of $\mathcal{G}$ on $E_{0}$ and on $E_{1}$ respectively as we have commented in Remark 1.2.12.

- $\Omega$ is a map that associates to each pair of composable arrows $\left(g_{2}, g_{1}\right) \in \mathcal{G}_{2}$ a linear $\operatorname{map} \Omega_{g_{2}, g_{1}}: E_{s\left(g_{1}\right)} \rightarrow C_{t\left(g_{2}\right)}$, and takes any $c_{0} \in C^{k-1}\left(\mathcal{G}, E_{0}\right)$ to $\Omega c_{0} \in C^{k+1}\left(\mathcal{G}, E_{1}\right)$, defined by

$$
\Omega c_{0}\left(g_{k+1}, \ldots, g_{1}\right):=\Omega_{g_{k+1}, g_{k}}\left(c\left(g_{k-1}, \ldots, g_{1}\right)\right)
$$

Remark 1.4.1. One can check that $D^{2}=0$ breaks into the following four equations:

- $D^{E_{0}} \partial+\partial D^{E_{1}}=0$,

- $\left(D^{E_{1}}\right)^{2}+\Omega \partial=0$

- $\left(D^{E_{0}}\right)^{2}+\partial \Omega=0$

- $D^{E_{1}} \Omega+\Omega D^{E_{0}}=0$.

In view of Proposition 1.3.4, the 4-tuple $\left(\partial, D^{E_{0}}, D^{E_{1}}, \Omega\right)$ corresponds to another 4-tuple $\left(\partial, \Delta^{E_{0}}, \Delta^{E_{1}}, \Omega\right)$ satisfying the structural equations as in Equation (1.3.2). Thus, we have an equivalent way to define a representation up to homotopy of a Lie groupoid on a graded vector bundle concentrated in degrees 0 and 1 , summarized in the following theorem:

Theorem 1.4.2. Let $\mathcal{G}_{1} \rightrightarrows \mathcal{G}_{0}$ be a Lie groupoid and $\mathcal{E}=E_{0} \oplus E_{1}$ be a graded vector bundle over $\mathcal{G}_{0}$. Then, there is a one-to-one correspondence between representations up to homotopy of $\mathcal{G}$ on $\mathcal{E}$ and 4-tuples $\left(\partial, \Delta^{E_{0}}, \Delta^{E_{1}}, \Omega\right)$, such that

- $\partial: E_{1} \rightarrow E_{0}$ is a vector bundle morphism,

- $\Delta^{E_{0}}: \mathcal{G} \rightarrow \operatorname{End}\left(E_{0}\right)$, and $\Delta^{E_{1}}: \mathcal{G} \rightarrow \operatorname{End}\left(E_{1}\right)$ are quasi-actions of $\mathcal{G}$,

- $\Omega: \mathcal{G}_{2} \rightarrow \operatorname{Hom}\left(\pi_{0}^{*} E_{0}, \pi_{2}^{*} E_{1}\right)$,

satisfying the following equations:

(i) $\Delta_{g_{1}}^{E_{0}} \circ \partial=\partial \circ \Delta_{g_{1}}^{E_{1}}$;

(ii) $\Delta_{g_{2}}^{E_{0}} \circ \Delta_{g_{1}}^{E_{0}}-\Delta_{g_{2} g_{1}}^{E_{0}}=-\partial \circ \Omega_{g_{2}, g_{1}}$;

(iii) $\Delta_{g_{2}}^{E_{1}} \circ \Delta_{g_{1}}^{E_{1}}-\Delta_{g_{2} g_{1}}^{E_{1}}=-\Omega_{g_{2}, g_{1}} \circ \partial$;

(iv) $\Delta_{g_{3}}^{E_{1}} \circ \Omega_{g_{2}, g_{1}}-\Omega_{g_{3} g_{2}, g_{1}}+\Omega_{g_{3}, g_{2} g_{1}}-\Omega_{g_{3}, g_{2}} \circ \Delta_{g_{3}}^{E_{0}}=0$

for every composable triple $\left(g_{3}, g_{2}, g_{1}\right) \in \mathcal{G}_{3}$. 


\subsubsection{The adjoint representation}

Some parts of the usual Lie theory of Lie groups and Lie algebras have natural extensions to the theory of Lie groupoids and Lie algebroids, for instance, we have a Lie functor from the category of Lie groupoids to the category of Lie algebroids, we also have integration theorems for objects and morphisms, but things already become different here, in fact, it is widely known that there are Lie algebroids which can not be integrated to any Lie groupoid, so that Lie's third theorem does not hold for every Lie algebroid. In fact, a complete characterisation of integrable Lie algebroids was obtained by M. Crainic and R. Fernandes in [15]. Also, when it comes to representation theory, in general, it is not possible to define an ordinary representation of a Lie groupoid on its Lie algebroid recovering the notion of adjoint representation of a Lie group on its Lie algebra. In this section, we will see how representations up to homotopy remedy this situation by allowing a notion of an adjoint representation, but to do that let's first recall the definition of Lie algebroid and the construction of a Lie algebroid from a Lie groupoid. Our main references for the basic theory on Lie groupoids and Lie algebroids are [43, 38]. For more details about the adjoint representation the reader can look at [3].

Definition 1.4.3. A Lie algebroid over a smooth manifold $M$ consists of a vector bundle $A$ over $M$, together with a vector bundle map $\rho: A \rightarrow T M$, called the anchor, covering the identity on $M$ and an $\mathbb{R}$-bilinear pairing [, ] : $\Gamma(A) \times \Gamma(A) \rightarrow \Gamma(A)$ satisfying

1. the Jacobi Identity: $[a,[b, c]]+[c,[a, b]]+[b,[c, a]]=0$, for every $a, b, c \in \Gamma(A)$ and

2. the Leibniz rule: $[a, f b]=f[a, b]+\rho(a)(f) b$, for every $a, b \in \Gamma(A)$ and $f \in C^{\infty}(M)$.

Example 1.4.4. If the base is just a point $M=\{*\}$, then a Lie algebroid over $M$ is, by definition, a Lie algebra.

Example 1.4.5. For any $M$, the tangent bundle $T M$ is a Lie algebroid over $M$ with the usual bracket of vector fields and the anchor $\rho=\mathrm{Id}$. The same is true for any involutive distribution inside $T M$, in this, case $\rho$ is the inclusion of the subbundle in $T M$.

There are many more examples, in particular there is a whole class of examples that is given by Lie algebroids coming from some Lie groupoid. Let's recall their definition now.

As usual, we think of an arrow $g \in \mathcal{G}_{1}$ with source $x$ and target $y$ as $y \stackrel{g}{\leftarrow} x$, this way it is easy to see that we can use $g$ to multiply on the right with arrows starting at $y$ and to multiply on the left with arrows ending at $x$. Hence, we define the right translation by $g$ as

$$
\begin{aligned}
R_{g}: s^{-1}(y) & \rightarrow s^{-1}(x) \\
h & \mapsto h g
\end{aligned}
$$


and the left translation by $g$ as

$$
\begin{aligned}
L_{g}: t^{-1}(x) & \rightarrow t^{-1}(y) \\
h & \mapsto g h
\end{aligned}
$$

In what follows, the differentials of $R_{g}$ and $L_{g}$ will be denoted by $r_{g}$ and by $l_{g}$ respectively. As one should expect, they will be used to define right and left invariant vector fields respectively.

If $\mathcal{G}_{1} \rightrightarrows \mathcal{G}_{0}$ is a Lie groupoid, we consider the vector bundle $A \mathcal{G}$ over $\mathcal{G}_{0}$ given by $A \mathcal{G}:=1^{*}\left(T^{s}(\mathcal{G})\right)$, where $T^{s}(\mathcal{G})=\operatorname{ker} \mathrm{d} s$ is the subbundle of $T \mathcal{G}_{1}$ tangent to the $s$-fibres and $1: \mathcal{G}_{0} \rightarrow \mathcal{G}_{1}$ is the unit map. Explicitly, the fibres of this vector bundle are given by

$$
(A \mathcal{G})_{x}=T_{1_{x}}\left(s^{-1}(x)\right) \text { for all } x \in M,
$$

the tangent spaces of the $s$-fibres at the units of the groupoid.

Definition 1.4.6. Let $\mathcal{G}_{1} \rightrightarrows \mathcal{G}_{0}$ be a Lie groupoid, then

$$
\mathfrak{X}^{s}(\mathcal{G}):=\left\{X \in \mathfrak{X}\left(\mathcal{G}_{1}\right) ; \text { such that } d s\left(X_{h}\right)=0, \forall h \in \mathcal{G}_{1} \text { and } r_{g}\left(X_{h}\right)=X_{h g}, \forall(h, g) \in \mathcal{G}_{2}\right\}
$$

is the space of right invariant vector fields on $\mathcal{G}$.

It is a standard fact that the space $\mathfrak{X}^{s}(\mathcal{G}) \subset \mathfrak{X}\left(\mathcal{G}_{1}\right)$ of right invariant vector fields on a Lie groupoid $\mathcal{G}$ is a Lie subalgebra for the bracket of vector fields on $\mathfrak{X}\left(\mathcal{G}_{1}\right)$.

Definition 1.4.7. Let $\mathcal{G}$ be a Lie groupoid, and $A \mathcal{G}$ the vector bundle defined above. Given any section $a \in \Gamma(A \mathcal{G})$ we define the right invariant vector field associated to $a$ by

$$
a^{r}(g):=d\left(R_{g}\right)\left(1_{s(g)}\right)(a(s(g)))=r_{g}(a(s(g))) .
$$

Note that, since $R_{g}$ takes $s$-fibres to $s$-fibres what we get is that $a^{r}$ is a vector field tangent to the $s$-fibres. And the property $r_{g}\left(X_{h}\right)=X_{h g}$ is automatically satisfied. Therefore, $a^{r}$ is in fact a well-defined right invariant vector field as in Definition 1.4.6.

Proposition 1.4.8. Let $\mathcal{G}$ be a Lie groupoid, and $A \mathcal{G}$ the vector bundle defined above. The map

$$
\Gamma(A \mathcal{G}) \rightarrow \mathfrak{X}^{s}(\mathcal{G}),
$$

associating a section $a \in \Gamma(A \mathcal{G})$ to the right invariant vector field $a^{r}$, is a linear isomorphism. Therefore, the space of sections $\Gamma(A \mathcal{G})$ can be turned into a Lie algebra, with the bracket induced from the bracket of vector fields in $\mathfrak{X}^{s}(\mathcal{G})$ via this linear isomorphism.

Proposition 1.4.9. Let $\mathcal{G}_{1} \rightrightarrows \mathcal{G}_{0}$ be a Lie groupoid, and $(A \mathcal{G},[]$,$) be the vector bundle A \mathcal{G}=$ $1^{*}(\operatorname{ker} d s)$ over $\mathcal{G}_{0}$, with the bracket $[$,$] on \Gamma(A \mathcal{G})$ as in Proposition 1.4.8. Then, defining the 
anchor map by $\partial=d t: A \mathcal{G} \rightarrow T \mathcal{G}_{0}$, we have that the triple $(A \mathcal{G},[],, \partial)$ is a Lie algebroid over $\mathcal{G}_{0}$. This Lie algebroid is called the Lie algebroid of the Lie groupoid $\mathcal{G}$.

Now, we can finally define the adjoint representation up to homotopy of a Lie groupoid $\mathcal{G}_{1} \rightrightarrows \mathcal{G}_{0}$. In order to do that, first we have to fix a linear splitting $\Sigma: s^{*} T \mathcal{G}_{0} \rightarrow T \mathcal{G}_{1}$ of the following short exact sequence of vector bundles over $\mathcal{G}_{1}$ :

$$
0 \longrightarrow t^{*} A \mathcal{G} \stackrel{r}{\longrightarrow} T \mathcal{G}_{1} \stackrel{d s}{\longrightarrow} s^{*} T \mathcal{G}_{0} \longrightarrow 0
$$

Such a splitting is called an Ehresmann connection on $\mathcal{G}$ if at the units it coincides with the canonical splitting, i.e. $\Sigma\left(e, 1_{x}\right)=1_{e}$.

Fixing such an Ehresmann connection $\Sigma$, we can build a representation up to homotopy of $\mathcal{G}$ on the graded vector bundle $\mathrm{Ad}:=T \mathcal{G}_{0} \oplus A \mathcal{G}$. Let us recall the formulas for the maps $\partial: A \mathcal{G} \rightarrow T \mathcal{G}_{0}, \Delta^{A \mathcal{G}}: \mathcal{G} \rightarrow \operatorname{End}(A \mathcal{G}), \Delta^{T \mathcal{G}_{0}}: \mathcal{G} \rightarrow \operatorname{End}\left(T \mathcal{G}_{0}\right), \Omega: \mathcal{G}^{(2)} \rightarrow$ $\operatorname{Hom}\left(\pi_{0}^{*} T \mathcal{G}_{0}, \pi_{2}^{*} A \mathcal{G}\right)$, satisfying the conditions of Theorem 1.4.2.

- $\partial: A \mathcal{G} \rightarrow T \mathcal{G}_{0}$ is given by the anchor of the Lie algebroid $\partial=\left.d t\right|_{A \mathcal{G}}$.

- $\Delta^{T \mathcal{G}_{0}}: \mathcal{G} \rightarrow \operatorname{End}\left(T \mathcal{G}_{0}\right)$ gives for every $g \in \mathcal{G}_{1}$ the linear map

$$
\begin{aligned}
\Delta_{g}^{T \mathcal{G}_{0}}: T_{s(g)} \mathcal{G}_{0} & \rightarrow T_{t(g)} \mathcal{G}_{0} \\
X_{s(g)} & \mapsto d t_{g}\left(\Sigma\left(X_{s(g)}, g\right)\right)
\end{aligned}
$$

- $\Delta^{A \mathcal{G}}: \mathcal{G} \rightarrow \operatorname{End}(A \mathcal{G})$ associates to each $g \in \mathcal{G}_{1}$ the linear map

$$
\begin{aligned}
\Delta_{g}^{A \mathcal{G}}: A \mathcal{G}_{s(g)} & \rightarrow A \mathcal{G}_{t(g)} \\
a_{s(g)} & \mapsto r_{g^{-1}}\left(l_{g}\left(a_{s(g)}\right)-\Sigma\left(d s\left(l_{g} a_{s(g)}\right), g\right)\right.
\end{aligned}
$$

- $\Omega_{g_{2}, g_{1}}: T_{s\left(g_{1}\right)} \mathcal{G}_{0} \rightarrow A \mathcal{G}_{t(g)}$ is such that $\Omega_{g_{2}, g_{1}}(v)$ is the unique element in $A \mathcal{G}_{t(g)}$ satisfying

$$
\begin{aligned}
r_{g_{2} g_{1}}\left(\Omega_{g_{2}, g_{1}}(v)\right) & =\Sigma\left(v, g_{2} g_{1}\right)-\Sigma\left(\Delta_{g_{1}}^{T \mathcal{G}_{0}}(v), g_{2}\right) \cdot \Sigma\left(v, g_{1}\right) \\
& =\Sigma\left(v, g_{2} g_{1}\right)-d m_{\left(g_{2}, g_{1}\right)}\left(\Sigma\left(\Delta_{g_{1}}^{T \mathcal{G}_{0}}, g_{2}\right), \Sigma\left(v, g_{1}\right)\right) .
\end{aligned}
$$

In other words, $\Omega_{g_{2}, g_{1}}(v)$ measures the failure for the Ehresmann connection $\Sigma$ to be multiplicative.

Definition 1.4.10. Let $\mathcal{G}_{1} \rightrightarrows \mathcal{G}_{0}$ be a Lie groupoid and $\Sigma$ an Ehresmann connection on $\mathcal{G}$. The graded vector bundle $\mathrm{Ad}$, together with the maps $\left(\partial, \Delta^{T \mathcal{G}_{0}}, \Delta^{A \mathcal{G}}, \Omega\right)$ defined above is called the adjoint representation up to homotopy of $\mathcal{G}$. In terms of differential graded 
modules, we have the complex $C\left(\mathcal{G}, \mathrm{Ad}_{\Sigma}\right){ }^{\bullet}$ with $C(\mathcal{G}, \mathrm{Ad})^{k}=C^{k+1}(\mathcal{G}, A \mathcal{G}) \oplus C^{k}\left(\mathcal{G}, T \mathcal{G}_{0}\right)$, together with a differential $D_{\Sigma}$, given by the formula

$$
D_{\Sigma}(u, v)=\left(\delta_{\Delta_{A \mathcal{G}}}(u)+\Omega \cdot v,-\delta_{\Delta^{T M}}(v)+\partial(u)\right) .
$$

We remark that, even though we have to choose an Ehresmann connection to be able to define the representation, different choices give rise to isomorphic representations [3]. Thus, in a sense the adjoint representation is not canonical, but it is up to isomorphisms.

\subsubsection{VB-groupoids}

In this section, we define the notion of a VB-groupoid, that in a very concise formulation may be defined as groupoid objects in the category of smooth real vector bundles. We recall the results of [28] where it is proved that VB-groupoids over a Lie groupoid $\mathcal{G}$ are the geometric counterpart of representations up to homotopy of $\mathcal{G}$ on two-term complexes. We also recall the definition of the VB-complex naturally associated to any VB-groupoid, its cohomology and the results showing that the VB-cohomology coincides with the cohomology of the two-term representation up to homotopy associated to it. All these constructions are very important for us since in the following chapters we want to understand how they can be generalized to the general setting of representations up to homotopy on any bounded complex, i.e. with a finite number of terms. So, we recall them here to give some background and also because they were the main inspiration for this project. Our main references for this section are $[28,19,12]$.

Definition 1.4.11. A VB-groupoid is a square

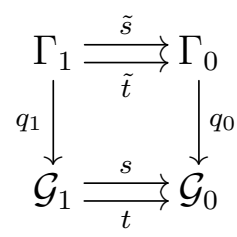

where $\Gamma$ and $\mathcal{G}$ are Lie groupoids, $\Gamma_{1} \rightarrow \mathcal{G}_{1}$ and $\Gamma_{0} \rightarrow \mathcal{G}_{0}$ are vector bundles, and all structural maps $(\tilde{s}, \tilde{t}, \tilde{m}, \tilde{i}, \tilde{1})$ of $\Gamma$ are vector bundle morphisms, covering the corresponding structural maps $(s, t, m, i, 1)$ of $\mathcal{G}$. We usually say that $\Gamma$ is a VB-groupoid over $\mathcal{G}$.

Definition 1.4.12. Let $\Gamma, \Gamma^{\prime}$ be VB-groupoids over $\mathcal{G}, \mathcal{G}^{\prime}$ respectively. Then, a morphism between them is a pair of Lie groupoid morphisms $\Phi: \Gamma \rightarrow \Gamma^{\prime}, \phi: \mathcal{G} \rightarrow \mathcal{G}^{\prime}$, such that $\Phi_{1}: \Gamma_{1} \rightarrow \Gamma_{1}^{\prime}, \Phi_{0}: \Gamma_{0} \rightarrow \Gamma_{0}^{\prime}$ are also vector bundle morphisms covering $\phi_{1}: \mathcal{G}_{1} \rightarrow \mathcal{G}_{1}^{\prime}, \phi_{0}:$ $\mathcal{G}_{0} \rightarrow \mathcal{G}_{0}^{\prime}$ respectively.

These definitions give rise to a category of VB-groupoids. We can also consider subcategories $\operatorname{VB}(\mathcal{G})$ formed by all VB-groupoids over a fixed $\mathcal{G}$. 
Example 1.4.13. Let $\mathcal{G}$ be any Lie groupoid. Then, applying the tangent functor to the manifolds $\mathcal{G}_{1}, \mathcal{G}_{0}$ and to all the structural maps $(s, t, m, 1, i)$ of $\mathcal{G}$ results in a VB-groupoid $T \mathcal{G}_{1} \rightrightarrows T \mathcal{G}_{0}$ over $\mathcal{G}$. This is called the tangent VB-groupoid of $\mathcal{G}$.

Example 1.4.14. If $\mathcal{G}_{1} \rightrightarrows \mathcal{G}_{0}$ has a representation of a vector bundle $E \rightarrow \mathcal{G}_{0}$, then, we can build an action groupoid $\mathcal{G} \ltimes E \rightrightarrows E$, with structural maps analogous to the ones in Example 1.1.6. This construction gives a VB-groupoid over $\mathcal{G}$.

Definition 1.4.15. Let $\Gamma_{1} \rightrightarrows E_{0}$ be a VB-groupoid over $\mathcal{G}_{1} \rightrightarrows \mathcal{G}_{0}$. Then, the vector bundle $E_{0} \rightarrow \mathcal{G}_{0}$ is called the side bundle, the vector bundle $E_{1}=\left.\operatorname{ker}\left(\tilde{s}: \Gamma_{1} \rightarrow E_{0}\right)\right|_{\mathcal{G}_{0}}$ is called the core bundle, and the map $\partial:=\tilde{t}: E_{1} \rightarrow E_{0}$ is called the anchor.

Let $r: t^{*} E_{1} \rightarrow \Gamma_{1}$ be the right multiplication by $0, r_{g}(c, g)=c \cdot 0_{g}$. Then, we can construct a short exact sequence of vector bundles over $\mathcal{G}_{1}$ :

$$
0 \rightarrow t^{*} E_{1} \stackrel{r}{\rightarrow} \Gamma_{1} \stackrel{\tilde{s}}{\rightarrow} s^{*} E_{0} \rightarrow 0
$$

called the core sequence of $\Gamma$.

As an example, the core of the tangent VB-groupoid $T \mathcal{G}$ is the Lie algebroid of $\mathcal{G}$, and the anchor is exactly the anchor of the Lie algebroid. Moreover, the core sequence of $T \mathcal{G}$ is the one we encountered in Equation (1.4.1), when defining the adjoint representation.

Definition 1.4.16. If $\Gamma_{1} \rightarrow E_{0}$ is a VB-groupoid over $\mathcal{G}$, then a cleavage is a linear splitting $\Sigma: s^{*} E_{0} \rightarrow \Gamma_{1}$ of the core sequence. Thus, given $y \stackrel{g}{\leftarrow} x$ an arrow in $\mathcal{G}_{1}$, and a vector $\left.e \in E_{0}\right|_{x}$, the arrow $\Sigma(e, g) \in \Gamma_{1}$ projects to $g$ and has source $\tilde{s}(\Sigma(e, g))=e$. The cleavage $\Sigma$ is called unital if $\Sigma\left(e, 1_{x}\right)=1_{e}$, and flat if $\Sigma\left(\tilde{t}\left(\Sigma\left(e, g_{1}\right)\right), g_{2}\right) \circ \Sigma\left(e, g_{1}\right)=\Sigma\left(e, g_{2} g_{1}\right)$.

Remark 1.4.17. Cleavages were also called horizontal lifts in the literature [28], and in the particular case of the tangent VB-groupoid, unital cleavages are also known as Ehresmann connections [3]. Every VB-groupoid admits a unital cleavage, but in general they may not admit a flat one.

As we have seen in the previous section, the choice of an Ehresmann connection on the tangent groupoid $T \mathcal{G}$ allows us to construct the adjoint representation up to homotopy. In general, for any choice of a unital cleavage $\Sigma$ on a VB-groupoid $\Gamma_{1} \rightrightarrows E_{0}$, we can build a two-term representation up to homotopy on the graded vector bundle $E_{0} \oplus E_{1}$ formed by the side bundle in degree zero and the core in degree one. This construction can be found in [28].

Also in ibid., there is a construction going in the other direction, that we will recall now. Let $\left(\partial, \Delta^{E_{1}}, \Delta^{E_{0}}, \Omega\right)$ be a representation up to homotopy of $\mathcal{G}$ on a two-term complex $E_{0} \oplus E_{1}$ as in Theorem 1.4.2. Then, we can construct a VB-groupoid

$$
\Gamma=t^{*} E_{1} \oplus s^{*} E_{0} \rightrightarrows E_{0}, \text { over } \mathcal{G}_{1} \rightrightarrows \mathcal{G}_{0},
$$


defined as a semidirect product groupoid. Concretely, denoting by $(c, g, e)$ an element of $t^{*} E_{1} \oplus s^{*} E_{0}$ over $g \in \mathcal{G}_{1}$, the structural maps of this Lie groupoid are given by:

- $\tilde{s}(c, g, e)=e$

- $\tilde{t}(c, g, e)=\partial(c)+\Delta_{g}^{E_{0}}(e)$

- $\tilde{1}(e)=\left(0_{x}^{E_{1}}, 1_{x}, e\right)$

- $\tilde{m}\left(\left(c_{2}, g_{2}, e_{2}\right),\left(c_{1}, g_{1}, e_{1}\right)\right)=\left(c_{2}+\Delta_{g_{1}}^{E_{1}}\left(c_{1}\right)-\Omega_{g_{2}, g_{1}}\left(e_{1}\right), g_{2} g_{1}, e_{1}\right)$;

- $(c, g, e)^{-1}=\left(-\Delta_{g^{-1}}^{E_{1}}(c)+\Omega_{g^{-1}, g}(e), g^{-1}, \partial(c)+\Delta_{g}^{E_{0}}(e)\right)$.

In ibid., it was proved that the previous construction gives a one-to-one correspondence between isomorphism classes. This was improved in [19] to an equivalence of categories.

Theorem 1.4.18. The semidirect product construction is functorial, thus it gives an equivalence of categories:

$$
\operatorname{Rep}_{2-\text { term }}^{\infty}(\mathcal{G}) \rightarrow \operatorname{VB}(\mathcal{G})
$$

between the category of two-term representations up to homotopy of $\mathcal{G}$ and the category of $V B$-groupoids over $\mathcal{G}$.

Not only this gives a correspondence at the level of objects and morphisms, but also it gives a new model for the cohomology with values in a two-term representation in terms of a cohomology naturally associated to any VB-groupoid, as it was shown in [28].

Definition 1.4.19. Let $\Gamma$ be a VB-groupoid over $\mathcal{G}$. Since $\Gamma$ is a Lie groupoid, there is associated to it the complex of differentiable cochains $\left(C^{\bullet}(\Gamma), \delta\right)$. Then, we can consider two subcomplexes of $C^{\bullet}(\Gamma)$. The first one is the linear complex of $\Gamma$, denoted $C_{\text {lin }}^{\bullet}(\Gamma)$, consisting of differentiable cochains $\phi: \Gamma_{n} \rightarrow \mathbb{R}$, that are linear when restricted to any fibre of the vector bundle $\Gamma_{n} \rightarrow \mathcal{G}_{n}$. The second one is called the VB-complex of the VBgroupoid, and consists of linear cochains called projectable, meaning that they satisfy the following two additional properties:

- $\phi\left(\gamma_{n}, \ldots, \gamma_{2}, 0_{g_{1}}\right)=0$, for every $\left(\gamma_{n}, \ldots, \gamma_{2}, 0_{g_{1}}\right) \in \Gamma_{n}$;

- $\delta(\phi)\left(\gamma_{n+1}, \ldots, \gamma_{2}, 0_{g_{1}}\right)=0$, for every $\left(\gamma_{n+1}, \ldots, \gamma_{2}, 0_{g_{1}}\right) \in \Gamma_{n+1}$.

The linear cohomology $H_{\text {lin }}^{\bullet}(\Gamma)$, and the VB-cohomology $H_{\mathrm{VB}}^{\bullet}(\Gamma)$ are defined as the cohomologies of the linear and of the VB-complex, respectively.

As shown in [28], the VB-cohomology is related to the cohomology of the representation up to homotopy in the following way: 
Theorem 1.4.20. Let be a Lie groupoid $\mathcal{G}$ with a representation up to homotopy on a two-term complex $\mathcal{E}=E_{0} \oplus E_{1}$, and $\Gamma$ be the VB-groupoid given by the semidirect product construction. Then, there is a canonical isomorphism

$$
C_{\text {diff }}\left(\mathcal{G}, \mathcal{E}^{*}\right)^{\bullet} \rightarrow C_{\mathrm{VB}}^{\bullet}(\Gamma)[1]
$$

between the differential complex of $\mathcal{G}$ with coefficients in the dual representation up to homotopy $\mathcal{E}^{*}$ and the VB-complex of $\Gamma$ shifted by one.

In [12], it was proved that the VB-complex and the linear complex of a VB-groupoid $\Gamma$ compute the same cohomology.

Theorem 1.4.21. The inclusion $C_{\mathrm{VB}}^{\bullet}(\Gamma) \hookrightarrow C_{\operatorname{lin}}^{\bullet}(\Gamma)$ is a quasi-isomorphism.

By combining the results of Theorem 1.4.20, Theorem 1.4.21, and the Morita invariance of the differentiable cohomology proved in [14], with some more effort the authors of [19] were able to prove the following result:

Theorem 1.4.22. Let $\phi: \mathcal{G} \rightarrow \mathcal{H}$ be a Morita map and $\mathcal{E}$ a two-term representation up to homotopy of $\mathcal{H}$. Then, the cohomologies $H_{\text {diff }}^{\bullet}\left(\mathcal{G}, \phi^{*} \mathcal{E}\right)$ and $H_{\text {diff }}^{\bullet}(\mathcal{H}, \mathcal{E})$ are canonically isomorphic.

This settled the Morita invariance of the cohomology of two-term representations up to homotopy. In the last chapter of this thesis, we will see how this can be generalized for representations with more than two terms. 



\section{Chapter 2}

\section{A brief introduction to simplicial objects}

In the last decade, Henriques[30], Pridham [45], Getzler [25], Zhu [50], Wolfson [49], among others, have developed the theory of Lie $n$-groupoids, that is: simplicial manifolds whose horn-filling maps are surjective submersions in all dimensions, and isomorphisms above dimension $n$. Also recently, see for instance [35, 41, 40], it has been proposed that symplectic Lie 2-groupoids are the objects integrating certain Courant Algebroids, that show us how important and natural these higher Lie groupoids are when one studies symplectic or Poisson geometry and their related fields. Examples of this higher groupoids include: Lie 0-groupoids that are precisely smooth manifolds and Lie 1-groupoids that are precisely nerves of Lie groupoids. In this chapter, we define what is a simplicial object in a category, we will be particularly concerned with the study of simplicial manifolds and simplicial vector bundles which are higher Lie groupoids and maps between them. Our main references for the classical simplicial homotopy theory are [27, 39], and for the theory on simplicial manifolds and higher Lie groupoids we follow mainly [30, 5, 49].

\subsection{Simplicial sets}

Definition 2.1.1. We define the category of finite ordinal numbers to be $\Delta$, the category whose objects are the sets $[n]:=\{0,1, \ldots, n\} \subset \mathbb{N}$ for $n \geq 0$, and the morphisms are non-decreasing functions between these sets.

In another perspective, we can look at the sets $[n]$ as categories themselves, because each $[n]$ is a totally ordered set:

$$
0 \rightarrow 1 \rightarrow 2 \rightarrow \cdots \rightarrow n
$$

thus we can put the category structure with only one arrow from $i$ to $j$ if and only if $i \leqslant j$. 
This way, the morphisms $\theta:[m] \rightarrow[n]$ are also functors (since functors must respect directions of arrows, such a functor is uniquely determined by a non-decreasing function).

Among all non-decreasing functions $\theta:[m] \rightarrow[n]$ in the category $\Delta$, there are very special ones, which can be used to generate all other morphisms, namely

$$
\begin{array}{lll}
\delta_{i}:[n-1] \rightarrow[n] & 0 \leq i \leq n & \text { called cofaces } \\
\sigma_{j}:[n+1] \rightarrow[n] & 0 \leq j \leq n & \text { called codegeneracies }
\end{array}
$$

where $\delta_{i}(j)=\left\{\begin{array}{ll}j & \text { if } j<i \\ j+1 & \text { if } j \geq i\end{array}\right.$ and $\sigma_{j}(i)=\left\{\begin{array}{ll}i & \text { if } i \leq j \\ i-1 & \text { if } i>j\end{array}\right.$ that is, in the category $\Delta$, $\delta_{i}$ is the unique injective map $[n-1] \rightarrow[n]$ that misses $i$ in the image, and $\sigma_{j}$ is the unique surjective map $[n+1] \rightarrow[n]$ that takes the value $j$ two times. Doing some computations we can see that these cofaces and codegeneracies are subject to the following identities, called cosimplicial identities

$$
\left\{\begin{array}{llrl}
\delta_{j} \delta_{i}=\delta_{i} \delta_{j-1} & & i<j \\
\sigma_{j} \delta_{i}=\delta_{i} \sigma_{j-1} & & i<j \\
\sigma_{j} \delta_{j}=1_{[n]}=\sigma_{j} \delta_{j+1} & & \\
\sigma_{j} \delta_{i}=\delta_{i-1} \sigma_{j} & & i>j+1 \\
\sigma_{j} \sigma_{i} & =\sigma_{i} \sigma_{j+1} & & i \leq j
\end{array}\right.
$$

Remark 2.1.2. These particular classes of morphisms are very important because it is known that any morphism $\theta:[m] \rightarrow[n]$ can be written as a composition of cofaces and/or codegeneracies([39]).

Now we are ready to define what is a simplicial set.

Definition 2.1.3. A simplicial set is a contravariant functor $X_{\bullet}: \Delta^{o p} \rightarrow$ Sets, where Sets denotes the category of sets.

Thus, in order to define a simplicial set $X_{\bullet}$, for each object $[n]$, we must assign a set of $\mathbf{n}$-simplices $X_{n}$, and for each order-preserving function $\theta:[m] \rightarrow[n]$ we must assign a function $X_{n} \rightarrow X_{m}$ (plus conditions making this association a functor). But, thanks to Remark 2.1.2, it suffices to write down sets $X_{n}$, for each $n \geq 0$, together with maps

$$
\begin{array}{lcl}
d_{i}: X_{n+1} \rightarrow X_{n} & 0 \leq i \leq n+1 & \text { called faces } \\
s_{j}: X_{n} \rightarrow X_{n+1} & 0 \leq j \leq n & \text { called degeneracies }
\end{array}
$$

where $d_{i}:=X_{\bullet}\left(\delta_{i}\right), s_{j}:=X_{\bullet}\left(\sigma_{j}\right)$, subject to the following conditions called simplicial 
identities

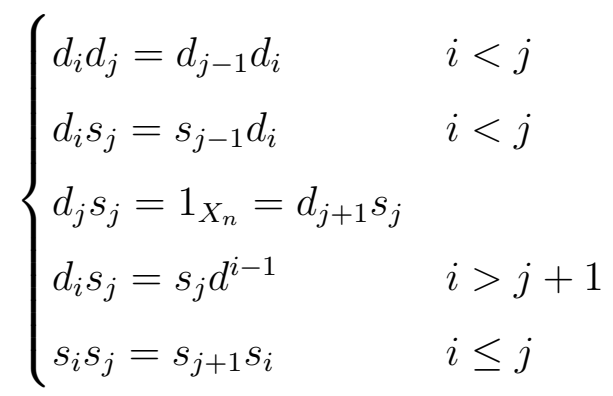

This is the classical way to write down the data for a simplicial set $X$.

Example 2.1.4. Let $\mathcal{G}_{1} \rightrightarrows \mathcal{G}_{0}$ be a groupoid. Then, we have a simplicial set denoted $\mathcal{G}_{\bullet}$ naturally associated with it, called the nerve of the groupoid, defined in the following way: the set of $n$-simplices is simply the set of strings of $n$ composable arrows

$$
\mathcal{G}_{n}:=\left\{\left(g_{n}, \ldots, g_{1}\right) \in \mathcal{G}_{1}^{n} \mid s\left(g_{i+1}\right)=t\left(g_{i}\right), 1 \leq i \leq n-1\right\}
$$

for every $n \geq 2$, the set of 1 -simplices is the set of arrows $\mathcal{G}_{1}$ and the set of 0 -simplices is the set of objects $\mathcal{G}_{0}$. The faces $d_{1}, d_{0}: \mathcal{G}_{1} \rightarrow \mathcal{G}_{0}$ are the source $s$ and target $t$ respectively, and the other face maps $d_{0}, d_{1}, \ldots, d_{n}: \mathcal{G}_{n} \rightarrow \mathcal{G}_{n-1}$ are defined by

$$
\left\{\begin{array}{l}
d_{0}\left(g_{n}, g_{n-1}, \ldots, g_{1}\right)=\left(g_{n}, \ldots, g_{2}\right) \\
d_{j}\left(g_{n}, g_{n-1}, \ldots, g_{1}\right)=\left(g_{n}, \ldots, g_{j+1} g_{j}, \ldots, g_{1}\right) \quad 1 \leq j \leq n-1 \\
d_{n}\left(g_{n}, g_{n-1}, \ldots, g_{1}\right)=\left(g_{n-1}, \ldots, g_{1}\right)
\end{array}\right.
$$

The degeneracy map $s_{0}: \mathcal{G}_{0} \rightarrow \mathcal{G}_{1}$ is the unit map 1 of the groupoid. More generally, $s_{0}, \ldots, s_{n-1}: \mathcal{G}_{n-1} \rightarrow \mathcal{G}_{n}$ are defined by

$$
\left\{\begin{array}{l}
s_{0}\left(g_{n-1}, \ldots, g_{1}\right)=\left(g_{n-1}, \ldots, g_{1}, 1_{s\left(g_{1}\right)}\right) \\
s_{j}\left(g_{n-1}, \ldots, g_{1}\right)=\left(g_{n-1}, \ldots, g_{j+1}, 1_{t\left(g_{j}\right)}, g_{j}, \ldots, g_{1}\right) \quad 1 \leq j \leq n-1 \\
s_{n-1}\left(g_{n-1}, \ldots, g_{1}\right)=\left(1_{t\left(g_{n-1}\right)}, g_{n-1}, \ldots, g_{1}\right)
\end{array}\right.
$$

\subsection{Simplicial spaces}

By the nature of Definition 2.1.3, it is natural to think of a definition of simplicial object in a more general category as follows.

Definition 2.2.1. A simplicial object in a category $\mathcal{C}$ is a contravariant functor $X$. : $\Delta^{o p} \rightarrow \mathcal{C}$

Remark 2.2.2. Usually we will drop the $\bullet$ mark and write just $X$ when referring to a simplicial object. 
By the same reasoning we did above, one can see that, in order to describe a simplicial object $X$ in a category $\mathcal{C}$ is suffices to prescribe the objects $X_{n}$ in $\mathcal{C}$, for each $n \geq 0$, and the face and degeneracy morphisms (that now are not just functions, but morphisms in $\mathcal{C}$ ) satisfying the simplicial identities of (2.1.2). If $X$ is a simplicial object in a concrete category (i. e. the objects of the category are sets), such that $X_{0}$ consists of only one point we will call $X$ a reduced simplicial object, for instance if $G$ is a group and we view it as a groupoid with only one object, then the nerve of $G$ is a reduced simplicial set.

In our work, we will be particularly concerned with simplicial groups, simplicial abelian groups, simplicial vector spaces, and simplicial manifolds, that are, by definition, simplicial objects in the category of groups Grp, abelian groups Ab, vector spaces Vect, and smooth manifolds Man, respectively.

Example 2.2.3. Let $\mathcal{G}_{1} \rightrightarrows \mathcal{G}_{0}$ be a Lie groupoid. Since the source and target maps $s$ and $t$ are submersions, it follows that each set of $n$-simplices $\mathcal{G}_{n}$ from the nerve of $\mathcal{G}$ has a structure of embedded submanifold of the product $\mathcal{G}_{1}^{n}$. Since the unit and multiplication maps of the Lie groupoid are smooth, it also follows that each face and degeneracy map from Example 2.1.4 is a smooth map. So we conclude that the nerve of a Lie groupoid $\mathcal{G}_{1} \rightrightarrows \mathcal{G}_{0}$ is a simplicial manifold.

Defining a simplicial object in $\mathcal{C}$ as a particular kind of functor into $\mathcal{C}$ also enables a conceptually clear way to define morphisms between simplicial objects.

Definition 2.2.4. Let $X, Y: \Delta^{o p} \rightarrow \mathcal{C}$ be two simplicial objects in $\mathcal{C}$. Then, a morphism $\phi: X \rightarrow Y$ is a natural transformation between these two functors.

Suppose we have a morphism $\phi: X \rightarrow Y$, then writing down what it means to be a natural transformation we see that it is equivalent to the following: for each $n \geq 0$ there is a morphism $\phi_{n}: X_{n} \rightarrow Y_{n}$ in $\mathcal{C}$, and these morphisms must commute with the face and degeneracy morphisms defining $X$ and $Y$, that is $\phi_{n} \circ d_{j}=d_{j} \circ \phi_{n+1}$ and $\phi_{n} \circ s_{j}=s_{j} \circ \phi_{n-1}$, for every $n$ and $j$ which make sense in the equations.

Definition 2.2.5. Let $\mathcal{C}$ be a category, then the classes of simplicial objects in $\mathcal{C}$ and morphisms between them as defined above give us a new category called the category of simplicial objects in $\mathcal{C}$, that is always denoted by $\mathbf{s} \mathcal{C}$. For instance, the categories of simplicial sets, simplicial abelian groups, simplicial vector spaces, and simplicial manifolds are denoted by sSets, sAb, sVect and sMan respectively.

In the study of homotopy theory of simplicial sets, there are special classes of simplicial sets, and special classes of morphisms between them, which play very important roles, e.g Kan-simplicial sets, fibrations and weak-equivalences. In what follows, we are are going to define all these concepts, and we will see what are the analogous ones when we transpose from simplicial sets to the setting of simplicial manifolds. For instance, when we interpret 
the notion of Kan-simplicial set into the smooth category, we will get the definition of an $\infty$-groupoid.

Definition 2.2.6. The standard $n$-simplex is the simplicial set $\Delta_{\text {e }}^{n}$ that, as a functor, is the contravariant functor in the category $\Delta$ represented by $[n]$. That is, $\Delta_{m}^{n}=\operatorname{hom}_{\Delta}([m],[n])$ for every $m \geq 0$, the face maps $d_{j}: \Delta_{m}^{n} \rightarrow \Delta_{m-1}^{n}$ are given by pre-composition with $\delta_{j}:[m-1] \rightarrow[m]$ in $\Delta$, and the degeneracy maps $s_{j}: \Delta_{m-1}^{n} \rightarrow \Delta_{m}^{n}$ are given by precomposition with $\sigma_{j}:[m] \rightarrow[m-1]$ in $\Delta$.

Definition 2.2.7. The $n$-simplex $\Delta^{n}$ contains as subcomplexes $\partial \Delta^{n}$ and $\Lambda_{k}^{n}$. The simplicial set $\partial \Delta^{n}$ is called the boundary of $\Delta^{n}$, and it is the smallest subcomplex of $\Delta^{n}$ containing all the faces $d_{j}\left(\Delta_{n}^{n}\right), 0 \leq j \leq n$. The simplicial set $\Lambda_{k}^{n}$, called the $k^{t h}$-horn of $\Delta^{n}$, is the smallest subcomplex of $\partial \Delta^{n}$ containing every face $d_{j}\left(\Delta_{n}^{n}\right), 0 \leq j \leq n$ except for $d_{k}\left(\Delta_{n}^{n}\right)$ (it is obtained from the boundary by removing the face opposed to the $k^{t h}$-vertex).

Definition 2.2.8. Let $X$ be a simplicial set. We define the $(n, k)$-horn of $X$ by

$$
\Lambda_{k}^{n} X:=\left\{\left(x_{0}, \ldots, \hat{x_{k}}, \ldots, x_{n}\right) \in X_{n-1}^{n} \mid d_{i}\left(x_{j}\right)=d_{j-1}\left(x_{i}\right), \forall i<j, i . j \neq k\right\},
$$

where the hat in the notation means that such term is omitted.

Note that, there is a one-to-one correspondence between the sets $\operatorname{Hom}_{\mathrm{sSets}}\left(\Lambda_{k}^{n}, X\right)$ and $\Lambda_{k}^{n} X$. This is sometimes useful and helps to understand better some conditions involving horn spaces.

Definition 2.2.9. Let $X$ be a simplicial set. The space defined by

$$
M_{n}(X):=\left\{\left(x_{0}, \ldots, x_{n}\right) \in X_{n-1}^{n+1} \mid d_{i}\left(x_{j}\right)=d_{j-1}\left(x_{i}\right), \forall i<j\right\}
$$

is called the $n^{\text {th }}$-matching space of $X$.

Note that, there is a one-to-one correspondence between the sets $\operatorname{Hom}_{\mathbf{s S e t s}}\left(\partial \Delta^{n}, X\right)$ and $M_{n}(X)$. In other words, an element in the matching space is a sequence of $(n-1)$ simplices satisfying the conditions that the faces of an $n$-simplex satisfy due to the simplicial identities. For instance, in degree one we have that $M_{1}(X)=\operatorname{Hom}_{\mathrm{sSets}}\left(\partial \Delta^{1}, X\right)=X_{0} \times$ $X_{0}$.

Definition 2.2.10. We say that a simplicial set $X$ is a Kan-simplicial set if it satisfies the following condition: for every $n \geq 0, k \leq n$, and for every element in the $(n, k)$-horn space $\left(x_{0}, \ldots, \hat{x_{k}}, \ldots, x_{n}\right) \in \Lambda_{k}^{n} X$, there is an $n$-simplex $x \in X_{n}$, such that $d_{j}(x)=x_{j}$ for every $j \neq k$. Or, in terms of diagrams, every morphism of simplicial sets $\alpha: \Lambda_{k}^{n} \rightarrow X$ can 
be extended to a map defined on $\Delta^{n}$. That is, there is a commutative diagram

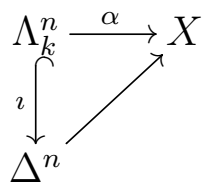

where the map $\imath$ is the inclusion.

Remark 2.2.11. The condition making a simplicial set into Kan simplicial set is usually called the extension condition, or the horn-filling condition in the literature. Moreover, Kansimplicial sets are also called Kan-complexes or fibrant simplicial sets.

Remark 2.2.12. We can rewrite the definition of Kan-simplicial sets as follows: $X$ is a Kan simplicial set if, and only if, the natural maps

$$
\left(d_{0}, \ldots, \hat{d}_{i}, \ldots, d_{n}\right): X_{n} \rightarrow \Lambda_{i}^{n} X
$$

are surjective for every $n \geq 0$ and $0 \leq i \leq n$. This is is the best way of describing Kansimplicial sets, since it can be generalized to the smooth setting by requiring these maps to be surjective submersions.

Definition 2.2.13. Let $X$ be a simplicial manifold. We say that $X$ is a Lie $\infty$-groupoid if each horn space $\Lambda_{i}^{n} X$ is a smooth manifold and the natural maps

$$
\left(d_{0}, \ldots, \hat{d}_{i}, \ldots, d_{n}\right): X_{n} \rightarrow \Lambda_{i}^{n} X
$$

are surjective submersions, for every $n \geq 0$, and $0 \leq i \leq n$. We also say that $X$ is a Lie $k$-groupoid if, moreover, these natural maps are diffeomorphims for every $n>k$, and $0 \leq i \leq n$. In other words, $k$-groupoids are $\infty$-groupoids where the horn filling is unique for every $n>k$.

By a standard argument, one can show that any simplicial group satisfies all the horn filling conditions, see for instance [27] for a proof.

Proposition 2.2.14. Let $H$ be a simplicial group, then the underlying simplicial set $H$ is a Kan-simplicial set.

Corollary 2.2.15. Every simplicial abelian group $H$ (in particular every simplicial vector space), is a Kan-simplicial set.

Remark 2.2.16. If $\mathcal{G}_{1} \rightrightarrows \mathcal{G}_{0}$ is a groupoid, then its nerve $\mathcal{G}_{\bullet}$ is a Kan-simplicial set with a unique horn filling for every $n>1$. 
In particular, the nerve of a group is a simplicial set satisfying all horn-filling conditions. One can see that the nerve construction gives an equivalence between the category of groups and the category of reduced Kan simplicial sets whose horns have a unique filling above dimension one. Moreover, one can show that the nerve extends to an equivalence between the category of groupoids and the category of Kan simplicial sets whose horns have a unique filling above dimension one. In terms of Lie groupoids the result is the following

Proposition 2.2.17. If $\mathcal{G}_{1} \rightrightarrows \mathcal{G}_{0}$ is a Lie groupoid, then its nerve $\mathcal{G}_{\bullet}$ is a Lie 1-groupoid. Moreover, if $X$ is a Lie 1-groupoid, then it is isomorphic to the nerve of a Lie groupoid.

Now we will look at some particular classes of morphisms between simplicial objects.

Definition 2.2.18. Let $\phi: X \rightarrow Y$ be a morphism of simplicial sets. We will say that $\phi$ is a Kan-fibration if for every commutative diagram (of solid arrows) of simplicial set morphisms

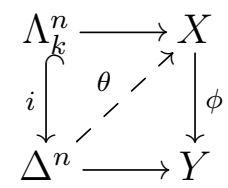

there is a simplicial set morphism $\theta: \Delta^{n} \rightarrow X$ (the dashed arrow) making the diagram commute.

The condition of $\phi: X \rightarrow Y$ being a Kan-fibration can be described in terms of elements in the following way: Given an $n$-tuple of $(n-1)$-simplices $\left(x_{0}, \ldots, \hat{x}_{i}, \ldots, x_{n}\right) \in \Lambda_{i}^{n} X$ and an $n$-simplex $y \in Y_{n}$, such that $d_{j}(y)=\phi\left(x_{j}\right)$ for every $j \neq i$, there must be an $n$-simplex $x \in X_{n}$, such that $d_{j}(x)=x_{j}$ for every $j \neq i$, and $\phi(x)=y$. Another way still is to ask the natural maps

$$
\left(\left(d_{0}, \ldots, \hat{d}_{i}, \ldots, d_{n}\right), \phi\right): X_{n} \mapsto \Lambda_{i}^{n} X \times_{\Lambda_{i}^{n} Y} Y_{n}
$$

to be surjective for every $n \geq 0$ and $0 \leq i \leq n$. This last way is good for us because it can be generalized to the smooth case requiring these maps to be surjective submersions.

Definition 2.2.19. Let $\phi: X \rightarrow Y$ be a morphism of simplicial manifolds. We say that $\phi$ is a smooth Kan-fibration if the fibre product $\Lambda_{i}^{n} X \times_{\Lambda_{i}^{n} Y} Y_{n}$ is a smooth manifold, and the natural maps

$$
\left(\left(d_{0}, \ldots, \hat{d}_{i}, \ldots, d_{n}\right), \phi\right): X_{n} \mapsto \Lambda_{i}^{n} X \times_{\Lambda_{i}^{n} Y} Y_{n}
$$

are surjective submersions, for every $n \geq 0$, and $0 \leq i \leq n$.

Remark 2.2.20. In what follows, we will refer to a smooth Kan-fibration simply as a fibration, if it is clear from the context that we are in the setting of simplicial manifolds. 
Remark 2.2.21. If $\phi: X \rightarrow Y$ is a morphism between simplicial manifolds where $Y$ is an $\infty$-groupoid, then the fibre product $\Lambda_{i}^{n} X \times_{\Lambda_{i}^{n} Y} Y_{n}$ is always a manifold since, in this case, the horn-filling map $Y_{n} \rightarrow \Lambda_{i}^{n}(Y)$ is a surjective submersion.

Remark 2.2.22. If $\phi: \mathcal{G} \rightarrow \mathcal{H}$ is a morphism of Lie groupoids, then the extended map between the nerves $\phi: \mathcal{G}_{\bullet} \rightarrow \mathcal{H}_{\bullet}$ is a smooth Kan-fibration if, and only if, $\phi$ is a fibration of Lie groupoids in the usual sense. That is, if both $\phi_{0}: \mathcal{G}_{0} \rightarrow \mathcal{H}_{0}$ and the map $\left(s, \phi_{1}\right): \mathcal{G}_{1} \rightarrow$ $\mathcal{G}_{0}{ }_{\phi_{0}} \times{ }_{s} \mathcal{H}_{1}$ are surjective submersions.

Theorem 2.2.23. ([27]) Let $\phi: X \rightarrow Y$ be a simplicial map between Kan-simplicial sets. Then, $\phi$ is a Kan-fibration and a weak equivalence if, and only if, $\phi$ has the right lifting property with respect to all inclusions $\partial \Delta^{n} \rightarrow \Delta^{n}, n \geq 0$, meaning that for every commutative diagram (of solid arrows) of simplicial set morphisms

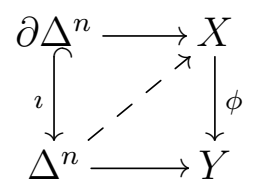

there is a simplicial set morphism $\theta: \Delta^{n} \rightarrow X$ (the dotted arrow) making the diagram commute.

In the literature of simplicial sets, a morphism $\phi: X \rightarrow Y$ between Kan-simplicial sets which is a Kan-fibration and a weak equivalence is called a trivial Kan-fibration. By the theorem above, we can describe the condition of being a trivial Kan-fibration in terms of a lifting property. And again, the lifting property condition can be described as the question of whether some maps are surjective or not.

Corollary 2.2.24. A simplicial map $\phi: X \rightarrow Y$ between Kan-simplicial sets is a trivial Kan-fibration if, and only if, the maps

$$
\left(\left(d_{0}, \ldots, d_{n}\right), \phi_{n}\right): X_{n} \mapsto M_{n}(X) \times_{M_{n}(Y)} Y_{n}
$$

are surjective for every $n \geq 0$.

In low degrees, the condition for $\phi$ to be a trivial fibration amounts to $X_{0} \stackrel{\phi_{0}}{\rightarrow} Y_{0}$ being surjective, and the same for $X_{1} \stackrel{\left(\left(d_{0}, d_{1}\right), \phi_{1}\right)}{\longrightarrow}\left(X_{0} \times X_{0}\right) \times_{Y_{0} \times Y_{0}} Y_{1}$.

\subsection{Hypercovers and equivalences between simplicial manifolds}

In this section, we introduce the notion of hypercovers between simplicial manifolds. These form a special class of simplicial manifold morphisms which are used to define the notion of 
equivalence between these spaces. We will also see that a hypercover between nerves of Lie groupoids is nothing else than a Morita fibration. Thus, the equivalence relation obtained from hypercovers generalizes that of Morita equivalence between Lie groupoids. Along the way, we also rewrite some propositions and lemmas from [5], that will be useful to us in this thesis, in the particular context of simplicial manifolds.

We arrive at the definition of a hypercover by transferring the concept of a trivial fibration to the smooth context.

Definition 2.3.1. Suppose that $\phi: X \rightarrow Y$ is a morphism of simplicial manifolds. Then, $\phi$ is called a hypercover if the fibre product $M_{n}(X) \times_{M_{n}(Y)} Y_{n}$ is a smooth manifold and the natural maps

$$
\left(\left(d_{0}, \ldots, d_{n}\right), \phi\right): X_{n} \mapsto M_{n}(X) \times_{M_{n}(Y)} Y_{n}
$$

are surjective submersions for every $n \geq 0$.

In low degrees, the condition for $\phi$ to be a hypercover amounts to $X_{0} \stackrel{\phi_{0}}{\longrightarrow} Y_{0}$ being a surjective submersion, and the same for $X_{1} \stackrel{\left(\left(d_{0}, d_{1}\right), \phi_{1}\right)}{\longrightarrow}\left(X_{0} \times X_{0}\right) \times_{Y_{0} \times Y_{0}} Y_{1}$. These conditions are readily similar to the ones in the definition of a Morita fibration between Lie groupoids (see Definition 1.1.15 and Definition 1.1.17).

One can show that Morita fibrations between Lie groupoids induce hypercovers between the nerves and vice-versa. We will get to that below, but first, we will introduce a few lemmas regarding fibrations and hypercovers. In [5], the authors prove a lot of lemmas concerning fibrations and hypercovers. But they consider simplicial objects and $n$ groupoids in what is called a descent category. Most of these lemmas work in the category of smooth manifolds Man with the exact same proofs, where we replace the class of maps called covers in the descent category by the class of surjective submersions (even though Man is not a descent category, because not all finite limits exist). We will see below some of these lemmas which give us important properties of fibrations and hypercovers. Similar discussions can also be found in [49, 30].

In what follows, whenever we refer to a $k$-groupoid, $k$ may be anything in $\mathbb{N} \cup\{\infty\}$.

Lemma 2.3.2 ([5] Lemma 3.11,pg 12). Suppose that $\phi: X \rightarrow Y$ is a fibration between Lie $k$-groupoids, then the natural map

$$
\beta:\left(\left(d_{0}, \ldots, \hat{d}_{i}, \ldots, d_{n}\right), \phi\right): X_{n} \mapsto \Lambda_{i}^{n} X \times_{\Lambda_{i}^{n} Y} Y_{n}
$$

must be a diffeomorphism for each $n>k$ and $0 \leq i \leq n$. 
Proof: We have the following commutative diagram in which the square is a pullback.

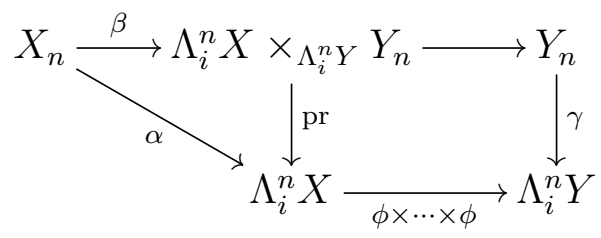

Now suppose that $n>k$, then the maps $\gamma$ and $\alpha$ are diffeomorphisms because $Y$ and $X$ are $k$-groupoids. Moreover, pr is a diffeomorphism since it is a pullback of a diffeomorphism. Therefore, by the commutativity of the triangle in the left, we have that $\operatorname{pr} \circ \beta=\alpha$ and so $\beta$ must be a diffeomorphism as well.

Lemma 2.3.3 ([5] Lemma 3.12,pg 12). Suppose that $\phi: X \rightarrow Y$ is a hypercover between Lie $k$-groupoids, then $\phi$ is a fibration.

Proof: Take $n \geq 1$ and $i \in\{0, \ldots, n\}$. We have the following commutative diagram in which the square is a pullback.

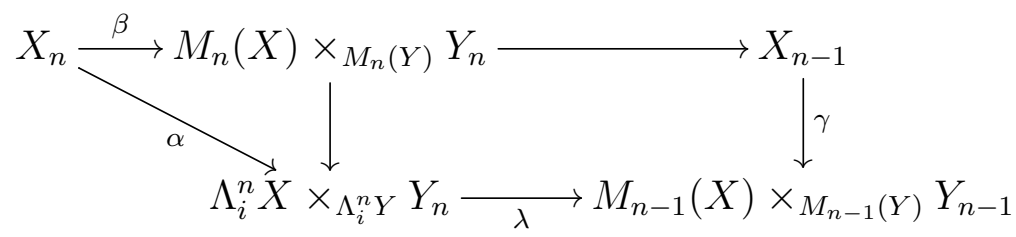

Using the hypothesis that $\phi$ is a hypercover we have that both $\gamma$ and $\beta$ are surjective submersions. Moreover, the pullback of $\gamma$ along $\lambda$ must be a surjective submersion because $\gamma$ is. Therefore by the commutativity of the left triangle we conclude that $\alpha$ is a surjective submersion. Since $\phi_{0}: X_{0} \rightarrow Y_{0}$ is a surjective submersion and we saw above that for arbitrary $n \geq 1$ and $0 \leq i \leq n$ the natural map $\alpha$ is a surjective submersion it follows that $\phi$ is a smooth Kan fibration.

Lemma 2.3.4. Suppose that $\phi: X \rightarrow Y$ is a hypercover between Lie $k$-groupoids, then the natural map

$$
\left(\left(d_{0}, \ldots, d_{n}\right), \phi\right): X_{n} \mapsto M_{n}(X) \times_{M_{n}(Y)} Y_{n}
$$

must be a diffeomorphism for each $n \geq k$.

Proof: By Lemma 2.3.3 we have that $\phi$ is a fibration. Suppose that $n>k$ and look at diagram (2.3.1), then by Lemma 2.3.5 it follows that the map $\alpha$ is a diffeomorphism, and since $\beta$ is a surjective submersion and the triangle commutes, we must have that $\beta$ is also a diffeomorphism in this case. It is only left to prove the case $n=k$. Now suppose that $n=k+1$ and look again at the diagram (2.3.1). We have seen above that in this case the maps $\alpha$ and $\beta$ are diffeomorphisms. Then it also follows that the pullback of $\gamma$ along $\lambda$ is 
a diffeomorphism. Therefore, using the fact that $\lambda$ is a surjective submersion, the pullback being a diffeomorphism implies that $\gamma$ must be a diffeomorphism.

Lemma 2.3.5 ([5] Lemma 3.17,pg 14). If $\phi: X \rightarrow Y$ is a fibration between Lie $k$-groupoids, and $f: Z \rightarrow Y$ is any morphism between $k$-groupoids, then in the pullback diagram

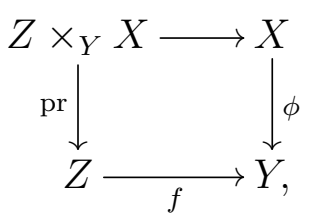

the simplicial manifold $Z \times_{Y} X$ is also a Lie $k$-groupoid and the projection pr is a fibration. Proof: Given $n>0$ and $i \in\{0, \ldots, n\}$ we have a pullback diagram

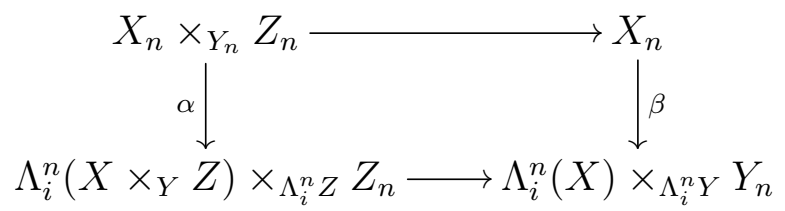

Since $\phi$ is a fibration we have that $\beta$ is a surjective submersion and it is a diffeomorphism for $n>k$, then it follows that the pullback map $\alpha$ is a surjective submersion and a diffeomorphism for $n>k$. There is another pullback diagram, given by

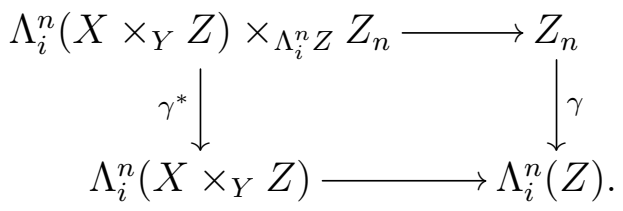

Since $Z$ is a $k$-groupoid we have that $\gamma$ is a surjective submersion and a diffeomorphism for $n>k$, then it follows that the pullback map $\gamma^{*}$ has the same properties. Therefore, the composition $\gamma^{*} \circ \alpha: X_{n} \times_{Y_{n}} Z_{n} \rightarrow \Lambda_{i}^{n}\left(X \times_{Y} Z\right)$ is a surjective submersion, and a diffeomorphism for $n>k$, which means that $X \times_{Y} Z$ is a $k$-groupoid. Moreover, since $\alpha$ is always a surjective submersion it follows that the pullback projection pr is a smooth Kan fibration.

Remark 2.3.6. Let $\phi: X \rightarrow Y$ be a fibration between Lie $k$-groupoids, $y$ be a 0 -simplex in $Y_{0}$, and for each $n \geq 1, s_{0}^{n}(y) \in Y_{n}$ be the degenerate $n$-simplex obtained by applying the degeneracy map $s_{0} n$-times. Then, we can think of $\{y\}$ as a simplicial manifold with just one $n$-simplex $s_{0}^{n}(y)$ for each $n$, and we define the fibre $F^{y}$ of $\phi$ over $y$ as the pullback of $\phi$ by the inclusion

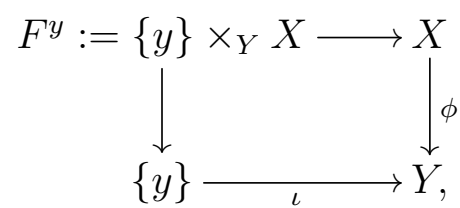


It follows from Lemma 2.3.5, that $F^{y}$ is also a simplicial manifold and a $k$-groupoid.

Lemma 2.3.7. If $\phi: X \rightarrow Y$ is a hypercover between Lie $k$-groupoids, and $f: Z \rightarrow Y$ is any morphism between $k$-groupoids, then in the pullback diagram

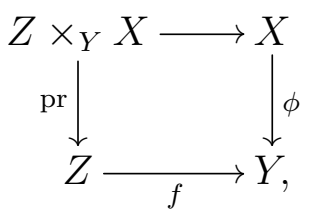

the pullback projection pr is a hypercover.

Proof: This follows from the pullback diagram below, because $\alpha$ being a surjective submersion implies that $\beta$ also is.

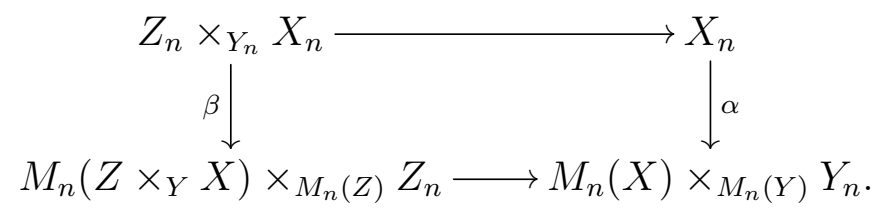

Using the fact that any hypercover is a fibration (Lemma 2.3.3), plus Lemma 2.3.5 and Lemma 2.3.7 we have the following corollary.

Corollary 2.3.8. If $\phi: X \rightarrow Y$ is a hypercover between Lie $k$-groupoids, and $f: Z \rightarrow Y$ is any morphism between $k$-groupoids, then in the pullback diagram

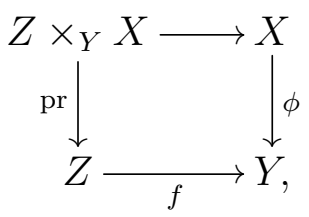

the simplicial manifold $Z \times_{Y} X$ is a Lie $k$-groupoid and the projection pr is a hypercover.

This finishes our series of lemmas concerning fibrations and hypercovers between $k$ groupoids. In what follows, we will always assume that our simplicial manifolds are at least $\infty$-groupoids, so that all these results apply.

Lemma 2.3.9. If $\mathcal{G}, \mathcal{H}$ are Lie groupoids and $\phi: \mathcal{G}_{\bullet} \rightarrow \mathcal{H}_{\bullet}$ is a morphism of simplicial manifolds, then the maps $\phi_{1}: \mathcal{G}_{1} \rightarrow \mathcal{H}_{1}$ and $\phi_{0}: \mathcal{G}_{0} \rightarrow \mathcal{H}_{0}$ define a morphism of Lie groupoids, and $\phi$ is the induced map by this Lie groupoid morphism.

Proof: Since the the face maps $d_{0}, d_{1}$ in degree one are the source and target of the groupoid, the map $d_{1}$ in degree two is the multiplication, the degeneracy $s_{0}$ is the unit, and a simplicial map commutes with every face and degeneracy map, it is straightforward that $\phi_{1}$ and $\phi_{0}$ define a morphism of Lie groupoids. 
Proposition 2.3.10. Let $\phi: \mathcal{G} \rightarrow \mathcal{H}$ be a morphism between Lie groupoids. Then, $\phi: \mathcal{G} \rightarrow \mathcal{H}$ is a Morita fibration if, and only if, the induced map $\phi: \mathcal{G}_{\bullet} \rightarrow \mathcal{H}_{\bullet}$ between the nerves is a hypercover.

Proof: Suppose that $\phi: \mathcal{G}_{\bullet} \rightarrow \mathcal{H}_{\bullet}$ is a hypercover. Then, by definition, we have surjective submersions

$$
\left(\left(d_{0}, \ldots, d_{n}\right), \phi\right): \mathcal{G}_{n} \mapsto M_{n}\left(\mathcal{G}_{\bullet}\right) \times_{M_{n}\left(\mathcal{H}_{\bullet}\right)} \mathcal{H}_{n}
$$

for every $n \geq 0$. Putting $n=0$, we get that the map $\phi_{0}: \mathcal{G}_{0} \rightarrow \mathcal{H}_{0}$ is a surjective submersion. When $n=1$, we get that the map $\left((s, t), \phi_{1}\right): \mathcal{G} \rightarrow \mathcal{G}_{0} \times \mathcal{G}_{0}{ }_{\phi_{0} \times \phi_{0}} \times{ }_{(s, t)} \mathcal{H}_{1}$ is a surjective submersion. Thus, to show that $\phi$ is a Morita fibration we only need to show that $\left((s, t), \phi_{1}\right)$ is injective.

Remember that $M_{2}\left(\mathcal{G}_{\bullet}\right)$ is the set of simplicial morphisms from $\partial \Delta^{2}$ to $\mathcal{G}_{\bullet}$. Then, it is the same as the set of triangles formed using arrows of $\mathcal{G}$

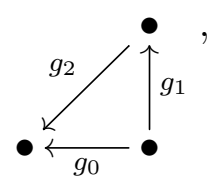

and if such a triangle commutes $\left(g_{0}=g_{2} g_{1}\right)$, it means that it is the boundary of the 2simplex $\left(g_{2}, g_{1}\right) \in \mathcal{G}_{2}$.

Suppose that $g_{0}$ and $g_{1}$ are arrows in $\mathcal{G}_{1}$, such that $\left((s, t), \phi_{1}\right)\left(g_{0}\right)=\left((s, t), \phi_{1}\right)\left(g_{1}\right)$. This implies that $s\left(g_{0}\right)=s\left(g_{1}\right), t\left(g_{0}\right)=t\left(g_{1}\right)$, and $\phi_{1}\left(g_{0}\right)=\phi_{1}\left(g_{1}\right)$. Thus, we have the triangle

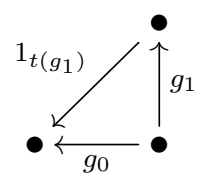

in $M_{2}\left(\mathcal{G}_{\bullet}\right)$, and by applying $\phi_{1}$ to it we get a commutative triangle

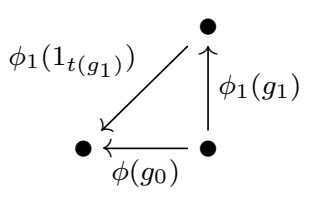

which corresponds to the 2 -simplex $\left(\phi_{1}\left(1_{t\left(g_{1}\right)}, \phi_{1}\left(g_{1}\right)\right) \in \mathcal{H}_{2}\right.$. In this way, we see that

$$
\left.\left(\left(1_{t\left(g_{1}\right)}, g_{0}, g_{1}\right)\right),\left(\phi\left(1_{t\left(g_{1}\right)}, \phi\left(g_{1}\right)\right)\right)\right) \in M_{2}\left(\mathcal{G}_{\bullet}\right) \times_{M_{2}\left(\mathcal{H}_{\bullet}\right)} \mathcal{H}_{1}{ }_{s} \times_{t} \mathcal{H}_{1} .
$$

Using the fact that, for $n=2$, the map

$$
\left(\left(d_{0}, d_{1}, d_{2}\right), \phi\right): \mathcal{G}_{1}{ }_{s} \times{ }_{t} \mathcal{G}_{1} \rightarrow M_{2}\left(\mathcal{G}_{\bullet}\right) \times_{M_{2}\left(\mathcal{H}_{\bullet}\right)} \mathcal{H}_{1}{ }_{s} \times{ }_{t} \mathcal{H}_{1}
$$


is surjective, we have that $\left(1_{t\left(g_{1}\right)}, g_{1}\right)$ is a 2 -simplex with boundary $\left(1_{t\left(g_{1}\right)}, g_{0}, g_{1}\right)$. In other words, $g_{0}=d_{1}\left(1_{t\left(g_{1}\right)}, g_{1}\right)=1_{t\left(g_{1}\right)} g_{1}=g_{1}$. Thus, we have proved that the surjective submersion $\left((s, t), \phi_{1}\right)$ is injective, so it is a diffeomorphism, and therefore $\phi$ is a Morita fibration.

The converse follows from the definition of a Morita fibration, and from the diagram in the proof of Lemma 2.3.3 using induction.

An equivalence relation between simplicial manifolds is defined in a similar way to the definition of Morita equivalence:

Definition 2.3.11. Two simplicial manifolds $X$ and $Y$ are equivalent if there is a third simplicial manifold $Z$ and hypercovers $\phi: Z \rightarrow X, \psi: Z \rightarrow Y$.

Proposition 2.3.12. The notion of equivalence between simplicial manifolds defined above is an equivalence relation.

Proof: Since for any manifold $X$, the identity map Id : $X \rightarrow X$ is a hypercover, it follows that $X$ is equivalent to $X$. It is also clear from the definition that this relation is symmetric. Let us prove that the relation is transitive. Suppose that $X$ is equivalent to $Y$ and $Y$ is equivalent to $Z$. Then, there are simplicial manifolds $W_{1}$ and $W_{2}$ together with hypercovers $\phi_{1}: W_{1} \rightarrow X, \psi_{1}: W_{1} \rightarrow Y$ and $\phi_{2}: W_{2} \rightarrow Y, \psi_{2}: W_{2} \rightarrow Z$. Hence, it follows from Lemma 2.3.7 that the fibre product $W_{1}{ }_{\psi_{1}} \times_{\phi_{2}} W_{2}$ is a simplicial manifold and the projections $\operatorname{pr}_{i}: W_{1} \psi_{1} \times_{\phi_{2}} W_{2} \rightarrow W_{i}$, for $i=1,2$, are hypercovers. Therefore, the hypercovers $\phi_{1} \circ \operatorname{pr}_{1}: W_{1 \psi_{1}} \times_{\phi_{2}} W_{2} \rightarrow X$ and $\psi_{2} \circ \operatorname{pr}_{2}: W_{1 \psi_{1}} \times_{\phi_{2}} W_{2} \rightarrow Z$ give the desired equivalence between $X$ and $Z$.

The equivalence classes of the equivalence relation given above are called Lie $n$-stacks. this extends the correspondence between Morita equivalence classes of Lie groupoids and differentiable stacks to the context of higher Lie groupoids.

\subsection{The de Rham cohomology of a simplicial manifold}

Given a simplicial manifold $X$, there is a natural complex $\left(C^{\bullet}(X), \delta\right)$ associated to $X$. This complex is defined as follows: for every $n \geq 0$ the space of $n$-cochains is

$$
C^{n}(X):=C^{\infty}\left(X_{n}, \mathbb{R}\right)
$$

and the differential in degree $n$ is

$$
\delta_{n}:=\sum_{j=0}^{n+1}(-1)^{j} d_{j}^{*},
$$

the sum with alternating signs of pullbacks by the face maps of $X$. It is a straightforward computation to show that $\delta^{2}=0$ because of the simplicial identities. The cohomology as- 
sociated to this complex is denoted $H_{\text {diff }}^{\bullet}(X)$ and is called the differentiable cohomology of $X$. For instance, if $X$ is the nerve of a Lie groupoid $\mathcal{G}$, the differentiable cohomology of the nerve is exactly the differentiable cohomology of $\mathcal{G}$ that we have seen in Chapter 1 . In this section, we investigate the question of whether the differentiable cohomology is an invariant under equivalences between simplicial manifolds. More precisely, we will show that for any hypercover $\phi: X \rightarrow Y$ between simplicial manifolds, the map induced in cohomology $\phi^{*}: H_{\text {diff }}^{n}(Y) \rightarrow H_{\text {diff }}^{n}(X)$ is an isomorphism for each $n \geq 0$. For this end, we will use a result of E. Getzler taken from [26] concerning the de Rham complex of a simplicial manifold.

For any simplicial manifold $X$ we can define a natural double complex, usually referred to as the Bott-Shulman-Stasheff complex, using the de Rham differential and the simplicial differential.

Definition 2.4.1. Let $X$ be a simplicial manifold, then its Bott-Shulman-Stasheff complex is defined by

$$
C^{p, q}(X)=\Omega^{q}\left(X_{p}\right) \text { for every } p, q \geq 0 \text {. }
$$

With horizontal differential being the simplicial differential

$$
\delta=\sum(-1)^{i} d_{i}^{*}: \Omega^{q}\left(X_{p}\right) \rightarrow \Omega^{q}\left(X_{p+1}\right)
$$

and vertical differential being the de Rham differential

$$
\mathrm{d}: \Omega^{q}\left(X_{p}\right) \rightarrow \Omega^{q+1}\left(X_{p}\right)
$$

The BSS-complex can be depicted by

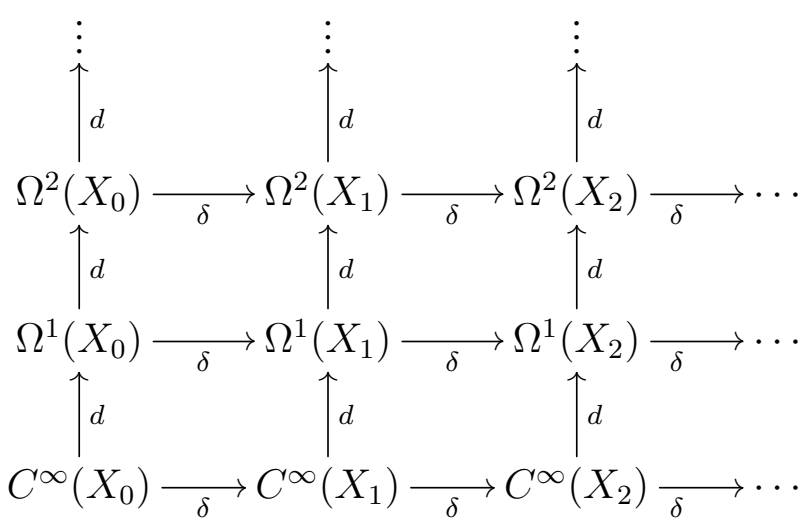

Note that, in the definition of this double complex, we used only the face maps to define the horizontal simplicial differential and we ignored the degeneracy maps. One can also define a normalized double complex by killing unwanted degeneracies. 
Definition 2.4.2. The normalized Bott-Shulman-Stasheff complex is defined by:

$$
\Omega^{q}\left(X_{p}\right)= \begin{cases}\Omega^{q}\left(X_{0}\right) ; & \text { if } p=0, q \geqslant 0 \\ \bigcap_{i=0}^{p-1} \operatorname{ker}\left(s_{i}^{*}: \Omega^{q}\left(X_{p}\right) \rightarrow \Omega^{q}\left(X_{p-1}\right)\right) ; & \text { if } p>0, q \geqslant 0 .\end{cases}
$$

The horizontal and vertical differentials are the same as before restricted to these subspaces. We only need to check that the simplicial differential is well-defined, that is $s_{i}^{*} \delta$ must be zero on $\Omega^{q}\left(X_{p}\right)$. This follows from the following computation:

$$
\begin{aligned}
s_{i}^{*} \delta= & s_{i}^{*}\left(d_{0}^{*}-d_{1}^{*}+\cdots+(-1)^{p+1} d_{p+1}^{*}\right) \\
= & \left(d_{0}^{*}-d_{1}^{*}+\cdots+(-1)^{i-1} d_{i-1}^{*}\right) s_{i-1}^{*} \\
& -\left((-1)^{i+1} d_{i+1}^{*}+\cdots+(-1)^{p} d_{p}^{*}\right) s_{i}^{*},
\end{aligned}
$$

where the last term clearly vanishes on $\mathbb{\Omega}^{q}\left(X_{p}\right)$.

As it is the case for any double complex, we obtain a total complex:

Definition 2.4.3. If $X$ is a simplicial manifold, then, the total complex $\operatorname{Tot}^{\bullet}\left(\Omega^{\bullet}\left(X_{\bullet}\right)\right)$ is obtained from the BSS-complex summing along diagonals

$$
\operatorname{Tot}^{n}\left(\mathbb{\Omega}^{\bullet}\left(X_{\bullet}\right)\right):=\bigoplus_{p+q=n} \mathbb{\Omega}^{q}\left(X_{p}\right)
$$

with the usual total differential $D_{X}:=\delta+(-1)^{p} \mathrm{~d}: \operatorname{Tot}^{n}\left(\Omega^{\bullet}\left(X_{\bullet}\right)\right) \rightarrow \operatorname{Tot}^{n+1}\left(\Omega^{\bullet}\left(X_{\bullet}\right)\right)$. Using the terminology of [26], this total complex will be called the de Rham complex of $X$, and the cohomology associated to it, denoted by $H_{\text {deRham }}^{\bullet}(X)$, will be called the de Rham cohomology of $X$.

More explicitly, an element of total degree $n$ in the total complex $\operatorname{Tot}^{\bullet}\left(\Omega^{\bullet}\left(X_{\bullet}\right)\right)$ consists of a collection of differential forms

$$
\left(\alpha_{0}, \alpha_{1}, \ldots, \alpha_{n}\right) \in \Omega^{n}\left(X_{0}\right) \oplus \Omega^{n-1}\left(X_{1}\right) \oplus \cdots \Omega^{0}\left(X_{n}\right)
$$

such that $s_{i}^{*} \alpha_{p}=0 \in \Omega^{n-p}\left(X_{p-1}\right)$ for all $0 \leqslant i \leqslant p-1$.

The differential $D_{X}$ is given by the formula

$$
\left(D_{X}\right)_{p}=\delta\left(\alpha_{p-1}\right)+(-1)^{p} \mathrm{~d}\left(\alpha_{p}\right) .
$$

We can also define a filtration of this double complex:

Definition 2.4.4. Let $X$ be a simplicial manifold, and $\Omega^{\bullet}\left(X_{\bullet}\right)$ be its normalized double 
complex. We define a filtration by rows:

$$
F_{i}^{p, q}\left(\Omega^{\bullet}\left(X_{\bullet}\right)\right)= \begin{cases}\Omega^{q}\left(X_{p}\right) ; & q \geq i \\ 0 ; & q<i .\end{cases}
$$

Thus, $\Omega^{q}\left(X_{p}\right)=F_{0}^{p, q}\left(\Omega^{\bullet}\left(X_{\bullet}\right)\right) \supset F_{1}^{p, q}\left(\Omega^{\bullet}\left(X_{\bullet}\right)\right) \supset \cdots$, where $F_{1}$ is a subcomplex of the double complex $\left(C^{p, q}(X), \delta, \mathrm{d}\right)$, where the first row is identically zero, $F_{2}$ is the subcomplex with first two rows equal zero, and so it goes.

Definition 2.4.5. For each double complex $F_{i}^{\bullet, \bullet}\left(\Omega^{\bullet}\left(X_{\bullet}\right)\right)$ of the the filtration described above, we consider its total complex, which will be denoted by $\operatorname{Tot}^{\bullet}\left(F_{i} \rrbracket^{\bullet}\left(X_{\bullet}\right)\right)$, having as differential the same $D_{X}$ as before restricted to this subspace.

Hence, an element of total degree $n$ in the total complex $\operatorname{Tot}^{\bullet}\left(F_{i} \rrbracket^{\bullet}\left(X_{\bullet}\right)\right)$ consists of a collection of differential forms

$$
\left(\alpha_{0}, \alpha_{1}, \ldots, \alpha_{n-i}\right) \in \Omega^{n}\left(X_{0}\right) \oplus \Omega^{n-1}\left(X_{1}\right) \oplus \cdots \Omega^{i}\left(X_{n-i}\right)
$$

such that $s_{i}^{*} \alpha_{p}=0 \in \Omega^{n-p}\left(X_{p-1}\right)$, for all $0 \leqslant i \leqslant p-1$.

In [26], it is proved that the cohomology of the complex $\operatorname{Tot}^{\bullet}\left(F_{i} \Omega^{\bullet}\left(X_{\bullet}\right)\right)$ is an invariant under taking hypercovers:

Theorem 2.4.6. Suppose $\phi: X \rightarrow Y$ is a hypercover between simplicial manifolds. Then, for each $i \geqslant 0$, the map induced via pullback between the total complexes

$$
\left.\phi^{*}:\left(\operatorname{Tot}^{\bullet}\left(F_{i} \rrbracket^{\bullet}\left(Y_{\bullet}\right)\right)\right), D_{Y}\right) \rightarrow\left(\operatorname{Tot}^{\bullet}\left(F_{i} \rrbracket^{\bullet}\left(X_{\bullet}\right)\right), D_{X}\right)
$$

is an isomorphism in cohomology.

Now, the result we were seeking about the hypercover invariance of the differentiable cohomology follows.

Theorem 2.4.7. Suppose that $\phi: X \rightarrow Y$ is a hypercover between simplicial manifolds. Then, the map induced via pullback between the complexes

$$
\phi^{*}:\left(C^{\bullet}(Y), \delta\right) \rightarrow\left(C^{\bullet}(X), \delta\right)
$$

induces an isomorphism in cohomology $\phi^{*}: H_{\mathrm{diff}}^{n}(Y) \rightarrow H_{\mathrm{diff}}^{n}(X)$, for each $n \geq 0$.

Proof: In this proof, in order to simplify the notation we will write $H^{k}\left(F_{1}(Z)\right)$ for the $k$-th group of cohomology of the complex $\left(\operatorname{Tot}^{\bullet}\left(F_{1} \Omega^{\bullet}\left(Z_{\bullet}\right)\right), D_{Z}\right)$ associated to a simplicial manifold $Z$. Note that, for any $Z$ the cochain complexes $\left(C^{\bullet}(Z), \delta\right),\left(\operatorname{Tot}^{\bullet}\left(F_{1} \Omega^{\bullet}(Z \bullet)\right), D_{Z}\right)$, 
and $\left(\operatorname{Tot}^{\bullet}\left(\Omega^{\bullet}(Z \bullet)\right), D_{Z}\right)$ fit in a short exact sequence of complexes

$$
0 \rightarrow\left(\operatorname{Tot}^{\bullet}\left(F_{1} \rrbracket^{\bullet}\left(Z_{\bullet}\right)\right), D_{Z}\right) \stackrel{\iota}{\rightarrow}\left(\operatorname{Tot}^{\bullet}\left(\Omega^{\bullet}\left(Z_{\bullet}\right)\right), D_{Z}\right) \stackrel{\pi}{\rightarrow}\left(C^{\bullet}(Z), \delta\right) \rightarrow 0
$$

where $\iota$ is the inclusion, and $\pi$ is the projection of the double complex to its first row.

If $\phi: X \rightarrow Y$ is any map of simplicial manifolds, it induces a morphism between short exact sequences:

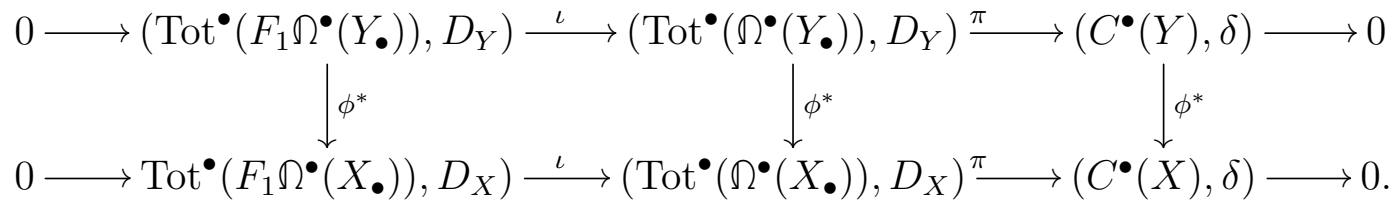

Since each short exact sequence of complexes gives rise to a long exact sequence in cohomology and the construction is natural on morphisms we get the following diagram in cohomology:

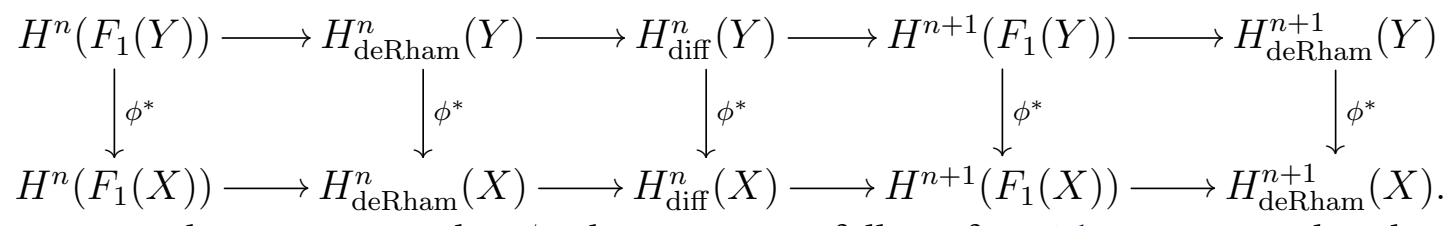

Using the assumption that $\phi$ is hypercover, it follows from Theorem 2.4.6 that the maps induced in cohomology $\phi^{*}: H^{k}\left(F_{1}(Y)\right) \rightarrow H^{k}\left(F_{1}(X)\right)$ and $\phi^{*}: H_{\text {deRham }}^{k}(Y) \rightarrow H_{\text {deRham }}^{k}(X)$ are isomorphisms, for every $k \geqslant 0$. Therefore, applying the five-lemma, we conclude that the map $\phi^{*}: H_{\text {diff }}^{n}(Y) \rightarrow H_{\text {diff }}^{n}(X)$ must be an isomorphism for each $n \geq 0$. 



\section{Chapter 3}

\section{The category of simplicial vector bundles}

In this chapter, we begin the study of simplicial vector bundles and morphisms between them. We define the notion of linear hypercover, which gives an appropriate notion of equivalence for simplicial vector bundles, analogous to the notion of equivalence between VB-groupoids in terms of VB-Morita maps. We also introduce the natural cochain complex associated to any simplicial vector bundle, consisting of differentiable cochains that take into account the linear structure, and we show that the cohomology of this complex is invariant under linear hypercovers.

Definition 3.0.1. A simplicial vector bundle is a simplicial object in the category of smooth vector bundles Vectb.

More explicitly, a simplicial vector bundle consists of two simplicial manifolds $V_{\bullet}$ and $X_{\bullet}$, such that for each $n \geqslant 0, V_{n} \rightarrow X_{n}$ is a vector bundle and each face and degeneracy map of $V$ is a vector bundle map covering the corresponding face or degeneracy map of $X$. Yet another way of saying this is to say that putting together all the bundle projection maps $p_{n}: V_{n} \rightarrow X_{n}$, we have a morphism of simplicial manifolds $p: V \rightarrow X$.

Whenever we have a simplicial vector bundle $p: V \rightarrow X$, we say that $p$ is the projection map, and that $V$ is a simplicial vector bundle over $X$.

The natural notion of morphisms between simplicial vector bundles is the following:

Definition 3.0.2. If $V$ and $W$ are simplicial vector bundles over $X$ and $Y$ respectively, then, a morphism between them is a pair of simplicial manifold morphisms $\Phi: V \rightarrow W$ and $\phi: X \rightarrow Y$, such that each $\Phi_{n}: V_{n} \rightarrow W_{n}$ is a vector bundle morphism covering $\phi_{n}: X_{n} \rightarrow Y_{n}$

Example 3.0.3. If $V$ is a simplicial vector space, then, we can consider $V$ as a simplicial vector bundle over the trivial simplicial manifold, which has only one $n$-simplex for each $n \geq 0$. 
One main class of examples of simplicial vector bundles is given by the nerves of VBgroupoids.

Example 3.0.4. If $\Gamma_{1} \rightrightarrows \Gamma_{0}$ is a VB-groupoid over $\mathcal{G}_{1} \rightrightarrows \mathcal{G}_{0}$, it follows that the nerve $\Gamma_{\bullet}$ of the Lie groupoid $\Gamma$ is a simplicial vector bundle over the nerve $\mathcal{G}_{\bullet}$ of $\mathcal{G}$.

Example 3.0.5. In the previous example, if the groupoid $\mathcal{G}$ is trivial $\mathcal{G}=\{*\} \rightrightarrows\{*\}$ then, a VB-groupoid over $\mathcal{G}$ is given by $\Gamma_{1} \rightrightarrows \Gamma_{0}$ where both $\Gamma_{1}$ and $\Gamma_{0}$ are just vector spaces and the structural maps are all linear. This is usually called a 2-vector space, its nerve is a simplicial vector space and in particular a simplicial vector bundle.

Example 3.0.6. Let $X$ be a simplicial manifold, then, we can apply the tangent functor to all manifolds $X_{n}$ and to all the face and degeneracy maps of $X$ to obtain the tangent simplicial manifold $T X$. This is an example of a simplicial vector bundle over $X$.

The first thing we noticed about the simplicial projection map $p: V \rightarrow X$, of a simplicial vector bundle, was that it is in fact a morphism between simplicial manifolds. But in the case where $V$ is a $\infty$-groupoid and $X$ is the nerve of a Lie groupoid, we can prove that $p$ is actually a smooth fibration.

Proposition 3.0.7. Suppose that $p: V_{\bullet} \rightarrow \mathcal{G}_{\bullet}$ is the simplicial projection map for a simplicial vector bundle $V$ over the nerve of a Lie groupoid $\mathcal{G}_{1} \rightrightarrows \mathcal{G}_{0}$, and that $V_{\bullet}$ is a Lie $\infty$-groupoid. Then, $p$ is a smooth Kan fibration.

Proof: For every $n \geq 2$ and $0 \leq i \leq n$ we can consider the following diagram of smooth manifolds where the square is a pullback

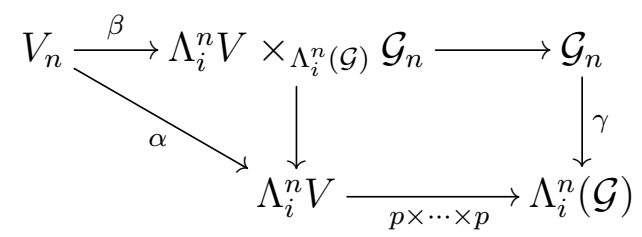

Since $\mathcal{G}_{\bullet}$ is the nerve of a Lie groupoid, we have that the natural map $\gamma$, in the diagram, is a diffeomorphism, and so it follows that its pullback is also a diffeomorphism. Thus, by commutativity of the triangle in the diagram, we conclude that $\beta$ is a surjective submersion if, and only if, $\alpha$ is a surjective submersion. But $\alpha$ is a surjective submersion because we are assuming that $V$ is a Lie $\infty$-groupoid. Hence, to finish the proof, it is only left to check the $n=1$ condition. Remember that the face map $d_{j}: V_{1} \rightarrow V_{0}(j=0$ or 1$)$ is a vector bundle map (and surjective submersion) covering the corresponding face map $d_{j}: \mathcal{G}_{1} \rightarrow \mathcal{G}_{0}$. Then, taking $i=0$ or $i=1$, and $j \neq i$, we have that the map

$$
\beta: V_{1} \rightarrow d_{j}^{*} V_{0}=V_{0}{ }_{p} \times_{d_{i}} \mathcal{G}_{1}=\Lambda_{i}^{1} V \times_{\Lambda_{i}^{1} \mathcal{G}} \mathcal{G}_{1} ; \text { given by } v \mapsto \beta(v)=\left(d_{j}(v), p_{1}(v)\right)
$$

is a surjective submersion, because it is a vector bundle map covering the identity of $\mathcal{G}_{1}$ and it is surjective over every fibre. 
Remark 3.0.8. Since the examples of simplicial vector bundles that we will be most interested in this thesis are always Lie $\infty$-groupoids, we may assume for now on that all simplicial vector bundles we encounter are higher groupoids.

Remark 3.0.9. Suppose that $p: V \rightarrow \mathcal{G}_{\bullet}$ is the projection map for a simplicial vector bundle $V$ over the nerve of a Lie groupoid $\mathcal{G}_{1} \rightrightarrows \mathcal{G}_{0}$. Then, by Lemma 2.3.5, for each $x \in \mathcal{G}_{0}$, we have a fibre $V^{x}$, which is a $k$-groupoid whenever $V$ is a $k$-groupoid. Explicitly, we have $V_{0}^{x}:=\left.V_{0}\right|_{x}$, and for every $n \geq 1, V_{n}^{x}=\left.V_{n}\right|_{\left(1_{x}, \ldots, 1_{x}\right)}$, with faces and degeneracies given by restrictions of faces and degeneracies of $V$. So in this case, each fibre is a simplicial vector space. For instance, if the simplicial vector bundle is the nerve of a VB-groupoid $\Gamma$, then, each fibre $\Gamma^{x}$ is also the nerve of a Lie groupoid, and thus, a 2-vector space, as in Example 3.0.5..

\subsection{A study of linear hypercovers}

In [19], the authors define the notion of VB-Morita maps between VB-groupoids:

Definition 3.1.1. A morphism between VB-groupoids $\left(\Phi: \Gamma \rightarrow \Gamma^{\prime}, \phi: \mathcal{G} \rightarrow \mathcal{G}^{\prime}\right)$ is a VB-Morita map if $\Phi$ is a Morita map of Lie groupoids.

This notion of Morita yields a natural notion of Morita equivalence between VB-groupoids. Moreover, also in [19], the authors give a characterization of VB-Morita maps in terms of base and fibre data:

Theorem 3.1.2. A morphism between VB-groupoids $\left(\Phi: \Gamma \rightarrow \Gamma^{\prime}, \phi: \mathcal{G} \rightarrow \mathcal{G}^{\prime}\right)$ is a VBMorita map if, and only if, $\phi$ is a Morita map and for each $x \in \mathcal{G}_{0}$ the map between fibres $\Phi^{x}: \Gamma^{x} \rightarrow \Gamma^{\phi_{0}(x)}$ is a Morita map.

By analogy with the VB-groupoid case, we introduce now the following class of morphisms between simplicial vector bundles:

Definition 3.1.3. Suppose that $(\Phi: V \rightarrow W, \phi: X \rightarrow Y)$ is a morphism of simplicial vector bundles. Then, we say that it is a linear hypercover if the map $\Phi$ is a hypercover.

In what follows, we study linear hypercovers between simplicial vector bundles. More precisely, we want to focus in the particular case where these bundles are over nerves of Lie groupoids, and we want to understand linear hypercovers in terms of fibre and base data.

Proposition 3.1.4. Suppose that $\phi: \mathcal{G} \rightarrow \mathcal{H}$ is a Morita fibration of Lie groupoids. Then, for any simplicial vector bundle $V$ over the nerve of $\mathcal{H}$, the pullback $\phi^{*} V$ is a simplicial vector bundle over the nerve of $\mathcal{G}$ and the natural map from $\phi^{*} V$ to $V$ is a linear hypercover. 
Proof: Since $p: V_{\bullet} \rightarrow \mathcal{H}_{\bullet}$ is a fibration it follows from Lemma 2.3.5 that $\phi^{*} V$ is a simplicial manifold, even if $\phi$ is not Morita. The pullback simplicial manifold $\phi^{*} V$ is a simplicial vector bundle, because each $\left(\phi^{*} V\right)_{n}=\phi_{n}^{*} V_{n}$ is a pullback vector bundle over $\mathcal{G}_{n}$. Moreover, being $\phi$ a Morita fibration, or a hypercover in the language of higher groupoids, it follows from Lemma 2.3.7 that the natural map $\phi^{*} V \rightarrow V$ is a linear hypercover covering $\phi$.

Proposition 3.1.5. Suppose that $V$ and $W$ are simplicial vector bundles over the nerve of a Lie groupoid $\mathcal{G}_{1} \rightrightarrows \mathcal{G}_{0}$. Let $\Phi: V \rightarrow W$ be a linear hypercover covering the identity id $: \mathcal{G}_{\bullet} \rightarrow \mathcal{G}_{\bullet}$. Then, for any $x \in \mathcal{G}_{0}$ the induced map between fibres $\Phi^{x}: V^{x} \rightarrow W^{x}$ is a hypercover.

Proof: Since $\Phi$ is hypercover, we have that, for each $n \geq 0$, the map

$$
\beta: V_{n} \rightarrow M_{n}(V) \times_{M_{n}(W)} W_{n} ; v \mapsto \beta(v)=\left(d_{0}(v), \ldots, d_{n}(v), \Phi_{n}(v)\right)
$$

is a surjective submersion. To prove that $\Phi^{x}$ is hypercover, we just need to show that the linear map

$$
\beta^{x}: V_{n}^{x} \rightarrow M_{n}\left(V^{x}\right) \times_{M_{n}\left(W^{x}\right)} W_{n}^{x} ; v \mapsto \beta^{x}(v)=\left(d_{0}(v), \ldots, d_{n}(v), \Phi_{n}^{x}(v)\right)
$$

is surjective for each $n \geqslant 0$. Let $\left(v^{0}, \ldots, v^{n}, w\right)$ be an element in $M_{n}\left(V^{x}\right) \times_{M_{n}\left(W^{x}\right)} W_{n}^{x}$, then, there is a $v \in V_{n}$ such that $\beta(v)=\left(v^{0}, \ldots, v^{n}, w\right)$, because $\beta$ is surjective. Since $\Phi_{n}: V_{n} \rightarrow W_{n}$ is a vector bundle map covering id $: \mathcal{G}_{n} \rightarrow \mathcal{G}_{n}, w \in W_{n}^{x}$ and $\Phi_{n}(v)=w$, we must have that $v \in V_{n}^{x}$. Therefore, it follows that $\beta^{x}$ is surjective.

\subsection{Hypercover invariance of the linear cohomology}

Following the same idea used to introduce the cohomology of a VB-groupoid, if we have $V_{\bullet}$ a simplicial vector bundle over any simplicial manifold $X_{\bullet}$, we can define a natural complex restricting our attention to differentiable cochains that take into account the linear structure. In this section, we will show that the cohomology obtained in this way is an invariant of $V$ under linear hypercovers.

Definition 3.2.1. For any simplicial vector bundle $V$, we define $\left(C_{\text {lin }}^{\bullet}(V), \delta\right)$ the subcomplex of $\left(C^{\bullet}(V), \delta\right)$ consisting of $n$-cochains $\phi: V_{n} \rightarrow \mathbb{R}$ that are linear when restricted to any fibre of the vector bundle $V_{n}$. These cochains are called linear cochains, and the cohomology associated to this complex is denoted $H_{\text {lin }}^{\bullet}(V)$ and is called the linear cohomology of $\mathrm{V}$.

Note that, we always have a map $P:\left(C^{\bullet}(V), \delta\right) \rightarrow\left(C_{\text {lin }}^{\bullet}(V), \delta\right)$, defined by $P(\phi)(v)=$ $\left.\frac{\mathrm{d}}{d t}\right|_{t=0} \phi(t v)$. In other words, $P(\phi)$ is the linearization of $\phi$ over each fibre and it follows that if $\phi$ is already a linear cochain, then $P(\phi)=\phi$. Therefore, $P$ is a natural projection map. 
Proposition 3.2.2. The projection $P:\left(C^{\bullet}(V), \delta\right) \rightarrow\left(C_{\text {lin }}^{\bullet}(V), \delta\right)$ is a cochain map.

Proof: We need to prove that $P(\delta(\phi))(v)=\delta(P(\phi))(v)$, for every $v \in V_{p}$, and for every $p \geq 0$. This follows from the fact that each face map of $V$ is linear on the fibres.

$$
\begin{aligned}
P(\delta(\phi))(v) & =P\left(\sum_{j=0}^{p+1}(-1)^{j} d_{j}^{*}(\phi)\right)(v) \\
& =\left.\frac{\mathrm{d}}{d t}\right|_{t=0}\left(\sum_{j=0}^{p+1}(-1)^{j} \phi d_{j}(t v)\right) \\
& =\left.\sum_{j=0}^{p+1}(-1)^{j} \frac{\mathrm{d}}{d t}\right|_{t=0} \phi\left(t d_{j}(v)\right) \\
& =\sum_{j=0}^{p+1}(-1)^{j} P(\phi) d_{j}(v) \\
& =\sum_{j=0}^{p+1}(-1)^{j} d_{j}^{*}(P(\phi))(v) \\
& =\delta(P(\phi))(v) .
\end{aligned}
$$

With this result and the one from Theorem 2.4.7, we have the following corollary:

Corollary 3.2.3. Let $V$ and $W$ be two simplicial vector bundles over $X$ and $Y$, respectively. If $\Phi: V \rightarrow W$ is a linear hypercover, then the map induced in cohomology $\Phi^{*}: H_{\text {lin }}^{p}(W) \rightarrow$ $H_{\text {lin }}^{p}(V)$ is an isomorphism, for every $p \geq 0$.

Proof: Since the natural projection $P:\left(C^{\bullet}(V), \delta\right) \rightarrow\left(C_{\text {lin }}^{\bullet}(V), \delta\right)$ is a cochain map, denoting by $K_{V}^{\bullet}$ the kernel of $P$, we have a direct sum decomposition of the cochain complex

$$
C^{\bullet}(V)=K_{V}^{\bullet} \oplus C_{\operatorname{lin}}^{\bullet}(V),
$$

that induces a direct sum decomposition at the cohomology level

$$
H^{\bullet}(V)=H^{\bullet}\left(K_{V}^{\bullet} \oplus H_{\operatorname{lin}}^{\bullet}(V)\right.
$$

Then, given $\Phi: V \rightarrow W$ a linear hypercover, we know from Theorem 2.4 .7 that it induces an isomorphism

$$
H^{\bullet}\left(K_{W}^{\bullet}\right) \oplus H_{\operatorname{lin}}^{\bullet}(W) \stackrel{\Phi^{*}}{\longrightarrow} H^{\bullet}\left(K_{V}^{\bullet}\right) \oplus H_{\operatorname{lin}}^{\bullet}(V) .
$$

Moreover, $\Phi^{*}$ has to preserve the direct sum decomposition because of its linearity, thus we get that

$$
H_{\text {lin }}^{\bullet}(W) \stackrel{\Phi^{*}}{\rightarrow} H_{\text {lin }}^{\bullet}(V)
$$


is an isomorphism as well.

\subsection{From representations up to homotopy to simplicial vector bundles}

Recall that a representation up to homotopy of $\mathcal{G}_{1} \rightrightarrows \mathcal{G}_{0}$ on a graded vector bundle $E_{\bullet}$ over $\mathcal{G}_{0}$ consists of a sequence of operators

$$
R_{m} \in \Gamma\left(\mathcal{G}_{m} ; \operatorname{Hom}^{(m-1)}\left(\pi_{0}^{*} E_{\bullet}, \pi_{m}^{*} E_{\bullet}\right)\right) \quad m \geq 0
$$

satisfying the structural equations that we have seen in chapter one Equation (1.3.2).

Also recall that $\mathcal{G}_{n}$ denotes the manifold of the $n$-simplices of the nerve of a Lie groupoid $\mathcal{G}_{1} \rightrightarrows \mathcal{G}_{0}$, and consists of chains of $n$ composable arrows $g=\left(x_{n} \stackrel{g_{n}}{\longleftarrow} x_{n-1} \cdots x_{1} \stackrel{g_{1}}{\longleftarrow} x_{0}\right)$. We denote by $\pi_{i}: \mathcal{G}_{n} \rightarrow \mathcal{G}_{0}$ the map that associates to each chain $g \in \mathcal{G}_{n}$ its $i$-th vertex, i.e. $\pi_{i}(g)=x_{i}$.

Starting with a representation up to homotopy $\left(R_{m}\right)_{m \geqslant 0}$ of $\mathcal{G}$ on a graded vector bundle $\mathcal{E}=\oplus_{j=0}^{l} E_{j}$, we will see now a result of [21], where the authors show how to build a simplicial vector bundle over the nerve of $\mathcal{G}$.

The vector bundle $V_{n}$ over $\mathcal{G}_{n}$ is defined by:

$$
V_{n}=\bigoplus_{[k] \stackrel{\alpha}{\rightarrow}[n]} \pi_{\alpha(k)}^{*} E_{k}
$$

where the sum is taken over all injective maps $\alpha:[k] \rightarrow[n]$ sending 0 to 0 .

Since each $V_{n}$ is a direct sum of $2^{n}$ components, a face map $d_{i}: V_{n} \rightarrow V_{n-1}$ is a $2^{n-1} \times 2^{n}$ matrix with entries $\left(d_{i}\right)_{\alpha \beta}$, depending on the indexes $[k] \stackrel{\alpha}{\rightarrow}[n]$ and $[l] \stackrel{\beta}{\rightarrow}[n-1]$, and analogously, a degeneracy $s_{j}: V_{n} \rightarrow V_{n+1}$ is a $2^{n+1} \times 2^{n}$ matrix with entries $\left(s_{j}\right)_{\alpha \beta}$, where $[k] \stackrel{\alpha}{\rightarrow}[n]$ and $[l] \stackrel{\beta}{\rightarrow}[n+1]$.

The degeneracies $s_{j}: V_{n} \rightarrow V_{n+1}$ and the faces $\left(d_{i}: V_{n} \rightarrow V_{n-1}\right)_{i \geqslant 1}$ are given by matrices whose entries are either 0 or Id, according to the following formulas:

$$
\left(s_{j}\right)_{\alpha \beta}=\left\{\begin{array}{ll}
\text { Id } & \alpha=\sigma_{j} \beta \\
0 & \text { otherwise }
\end{array} \quad\left(d_{i}\right)_{\alpha \beta}= \begin{cases}\text { Id } & \alpha=\delta_{i} \beta \\
0 & \text { otherwise }\end{cases}\right.
$$

The definition of the face map $d_{0}: V_{n} \rightarrow V_{n-1}$ follows the same notation used for the other face maps, and it is given by a matrix whose entries are either $0, \pm \mathrm{Id}$, or $\pm R_{m}$ for some $m$. To give the precise formula, let us introduce the following notation. If $\theta$ : $[m] \rightarrow[n]$ is an order map, the map $\bar{\theta}:[m+1] \rightarrow[n+1]$ is defined by $\bar{\theta}(0)=0$ and 
$\bar{\theta}(i+1)=\theta(i)+1$, for all $i \geqslant 0$. Then, the face map $d_{0}$ is given by:

$$
\left(d_{0}\right)_{\alpha \beta}= \begin{cases}(-1)^{j-1} \mathrm{Id} & \alpha^{\prime}=\bar{\beta}^{\prime} \delta_{j} \\ (-1)^{l} R_{l-k+1}^{\left.g \circ \bar{\beta}^{\prime}\right|_{[k, l+1]}} & \alpha^{\prime}=\left.\bar{\beta}^{\prime}\right|_{[0, k]} \\ 0 & \text { otherwise }\end{cases}
$$

The following is a theorem of [21].

Theorem 3.3.1. Given a representation up to homotopy $\left(R_{m}\right)_{m \geqslant 0}$ of $\mathcal{G}$ on $E_{\bullet}$, the semi-direct product, given by the vector bundles $V_{n}$ together with the structure maps $d_{i}: V_{n} \rightarrow V_{n-1}$ and $\sigma_{j}: V_{n} \rightarrow V_{n+1}$ defined above, is a simplicial vector bundle over the nerve of $\mathcal{G}$. Moreover, $V$. is a higher Lie groupoid. 



\section{Chapter 4}

\section{Morita invariance of the cohomology with values in a representation up to homotopy}

In this chapter, we will study the simplicial vector bundles coming from representations up to homotopy more closely. To be precise, we will study some natural complexes arising from these simplicial vector bundles and the cohomologies associated with them. Our main results are the following: we show that the linear cohomology of these simplicial vector bundles is a Morita invariant, we identify a subcomplex of the linear complex that computes the cohomology of a representation up to homotopy, and in the last part of the chapter we use these results to settle the Morita invariance of the cohomology with coefficients in a representation up to homotopy. This provides a generalisation of some results obtained by [28], [12] and [19], in the context of two-term representations up to homotopy, for more general representations on any bounded complex, and is part of joint work with M. del Hoyo and C. Ortiz.

\subsection{The complex of projectable cochains}

Let $\left(C(\mathcal{G}, \mathcal{E})^{\bullet}, D\right)$ be a representation up to homotopy on a $(l+1)$-term complex

$$
0 \rightarrow E_{l} \rightarrow E_{l-1} \rightarrow \cdots \rightarrow E_{1} \rightarrow E_{0} \rightarrow 0
$$

with associated simplicial vector bundle $V_{\bullet} \stackrel{p}{\rightarrow} \mathcal{G} \bullet$ over the nerve. Consider $\left(C\left(\mathcal{G}, \mathcal{E}^{*}\right)^{\bullet}, D^{\prime}\right)$ the dual representation up to homotopy on the complex

$$
0 \rightarrow E_{0}^{*} \rightarrow E_{1}^{*} \rightarrow \cdots \rightarrow E_{l-1}^{*} \rightarrow E_{l}^{*} \rightarrow 0
$$


where each $E_{j}^{*}$ has degree $l-j$. Then, we can define a map

$$
\lambda:\left(C\left(\mathcal{G}, \mathcal{E}^{*}\right)^{\bullet-l}, D^{\prime}\right) \rightarrow C_{\operatorname{lin}}^{\bullet}(V)
$$

in the following way: $\left(c_{0}, c_{1}, \ldots, c_{l}\right) \in C\left(\mathcal{G}, \mathcal{E}^{*}\right)^{p-l}=C^{p}\left(\mathcal{G}, E_{0}^{*}\right) \oplus C^{p-1}\left(\mathcal{G}, E_{1}^{*}\right) \oplus \cdots \oplus$ $C^{p-l}\left(\mathcal{G}, E_{l}^{*}\right)$ is mapped to $\lambda\left(c_{0}, \ldots, c_{l}\right): V_{p} \rightarrow \mathbb{R}$, a linear map on each fibre of $V_{p}$, given by

$$
\lambda\left(c_{0}, \ldots, c_{l}\right)(v):=\sum_{k=0}^{l}(-1)^{k} c_{k}\left(g_{k+1}^{-1}, \ldots, g_{p}^{-1}\right)\left(v_{\iota_{k}}\right),
$$

where $v \in V_{p}$ is any vector with projection $p(v)=\left(g_{p}, \ldots, g_{1}\right) \in \mathcal{G}_{p}$, and $\iota_{k}:[k] \rightarrow[p]$ is the inclusion for any $k \geqslant 0$.

Proposition 4.1.1. The map $\lambda:\left(C\left(\mathcal{G}, \mathcal{E}^{*}\right)^{\bullet-l}, D^{\prime}\right) \rightarrow\left(C_{\text {lin }}^{\bullet}(V), \bar{\delta}\right)$, defined above, is an injective morphism of cochain complexes, where $\bar{\delta}=(-1)^{l} \delta$.

Proof: The map $\lambda$ is injective because $\lambda\left(c_{0}, \ldots, c_{l}\right)(v)=0$, for every $v \in V_{p}$, implies that each of the sections $c_{0}, \ldots, c_{l}$ is identically zero. In order to prove that $\lambda$ is a cochain map, we only need to show that the following square commutes

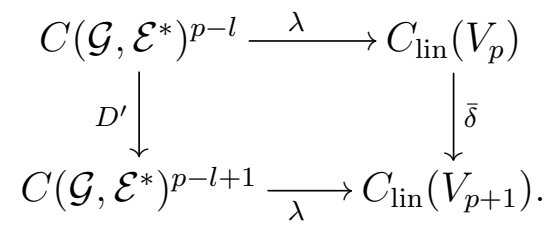

Since $C\left(\mathcal{G}, \mathcal{E}^{*}\right)^{p-l}=\oplus_{j=0}^{l} C^{p-j}\left(\mathcal{G}, E_{j}^{*}\right)$ is a direct sum, it is sufficient to check that the diagram commutes for a generic element $c_{j} \in C^{p-j}\left(\mathcal{G}, E_{j}^{*}\right)$.

Lets compute first $\lambda \circ D^{\prime}\left(\left(c_{j}\right)\right)(v)$, for any $v \in V_{p+1}$. Note that, $D^{\prime}\left(c_{j}\right)$ has components

$$
\left(D^{\prime}\left(c_{j}\right)_{0}, D^{\prime}\left(c_{j}\right)_{1}, \ldots, D^{\prime}\left(c_{j}\right)_{j+1}\right) \in C^{p+1}\left(\mathcal{G}, E_{0}^{*}\right) \oplus C^{p}\left(\mathcal{G}, E_{1}^{*}\right) \oplus \ldots C^{p-j}\left(\mathcal{G}, E_{j+1}^{*}\right)
$$

Moreover, as we have seen in Proposition 1.3.3 and Proposition 1.3.4, for each $i \neq j$, $D^{\prime}: C^{p-j}\left(\mathcal{G}, E_{j}^{*}\right) \rightarrow C^{p+1-i}\left(\mathcal{G}, E_{i}^{*}\right)$ corresponds to a left composition with one of the operators of the representation up to homotopy, that is

$$
\begin{aligned}
D^{\prime}\left(c_{j}\right)_{i}\left(g_{i+1}^{-1}, \ldots, g_{p+1}^{-1}\right) & =(-1)^{l-j} R_{j-i+1}^{*}\left(g_{i+1}^{-1}, \ldots, g_{j+1}^{-1}\right) c_{j}\left(g_{j+2}^{-1}, \ldots, g_{p+1}^{-1}\right) \\
& =(-1)^{l-j}(-1)^{j-i} c_{j}\left(g_{j+2}^{-1}, \ldots, g_{p+1}^{-1}\right) \circ R_{j-i+1}\left(g_{j+1}, \ldots, g_{i+1}\right)
\end{aligned}
$$

As for the case $i=j$, we have that $(-1)^{l-j} D^{\prime}: C^{p-j}\left(\mathcal{G}, E_{j}^{*}\right) \rightarrow C^{p+1-j}\left(\mathcal{G}, E_{j}^{*}\right)$ is a derivation that raises the cocycle degree by 1 and so it corresponds to the quasi action $R_{1}^{*}$ via 


$$
\begin{aligned}
(-1)^{l-j} D\left(c_{j}\right)_{j}\left(g_{j+1}^{-1}, \ldots, g_{p+1}^{-1}\right)= & R_{1}^{*}\left(g_{j+1}^{-1}\right) c_{j}\left(g_{j+2}^{-1}, \ldots, g_{p+1}^{-1}\right) \\
& +\sum_{n=0}^{p-j+1}(-1)^{p-j+1-n} c_{j}\left(d_{n}\left(g_{j+1}^{-1}, \ldots, g_{p+1}^{-1}\right)\right) \\
= & c_{j}\left(g_{j+2}^{-1}, \ldots, g_{p+1}^{-1}\right) \circ R_{1}\left(g_{j+1}\right) \\
& +\sum_{k=j+1}^{p+1}(-1)^{k-j} c_{j}\left(g_{j+1}^{-1}, \ldots, g_{k}^{-1} g_{k+1}^{-1}, \ldots, g_{p+1}^{-1}\right)
\end{aligned}
$$

Hence, we have that

$$
\begin{aligned}
\lambda\left(D^{\prime}\left(c_{j}\right)\right)(v)= & (-1)^{0} D^{\prime}\left(c_{j}\right)_{0}\left(v_{\iota 0}\right)+\cdots+(-1)^{j+1} D^{\prime}\left(c_{j}\right)_{j+1}\left(v_{\iota_{j+1}}\right) \\
= & (-1)^{0}(-1)^{l} c_{j}\left(g_{j+2}^{-1}, \ldots, g_{p+1}^{-1}\right) \circ R_{j+1}\left(g_{j+1}, \ldots, g_{2}, g_{1}\right)\left(v_{\iota}\right) \\
& +(-1)^{1}(-1)^{l-1} c_{j}\left(g_{j+2}^{-1}, \ldots, g_{p+1}^{-1}\right) \circ R_{j}\left(g_{j+1}, \ldots, g_{2}\right)\left(v_{\iota 1}\right)+\cdots \\
& +(-1)^{j+1}(-1)^{l-j-1} c_{j}\left(g_{j+2}^{-1}, \ldots, g_{p+1}^{-1}\right) \circ R_{0}\left(v_{\iota_{j+1}}\right) \\
& +(-1)^{j}(-1)^{l-j} c_{j}\left(g_{j+2}^{-1}, \ldots, g_{p+1}^{-1}\right) R_{1}\left(g_{j+1}\right)\left(v_{\iota j}\right) \\
& +(-1)^{j} \sum_{k=j+1}^{p+1}(-1)^{k-l} c_{j}\left(g_{j+1}^{-1}, \ldots, g_{k}^{-1} g_{k+1}^{-1} \ldots, g_{p+1}^{-1}\right)\left(v_{\iota j}\right) .
\end{aligned}
$$

and adjusting all the signs we get

$$
\begin{aligned}
\lambda\left(D^{\prime}\left(c_{j}\right)\right)(v)= & (-1)^{l} c_{j}\left(g_{j+2}^{-1}, \ldots, g_{p+1}^{-1}\right) \circ R_{j+1}\left(g_{j+1}, \ldots, g_{2}, g_{1}\right)\left(v_{\iota_{0}}\right) \\
& +(-1)^{l} c_{j}\left(g_{j+2}^{-1}, \ldots, g_{p+1}^{-1}\right) \circ R_{j}\left(g_{j+1}, \ldots, g_{2}\right)\left(v_{\iota_{1}}\right)+\cdots \\
& +(-1)^{l} c_{j}\left(g_{j+2}^{-1}, \ldots, g_{p+1}^{-1}\right) \circ R_{0}\left(v_{\iota_{j+1}}\right) \\
& +(-1)^{l} c_{j}\left(g_{j+2}^{-1}, \ldots, g_{p+1}^{-1}\right) R_{1}\left(g_{j+1}\right)\left(v_{\iota_{j}}\right) \\
& +\sum_{k=j+1}^{p+1}(-1)^{k-l+j} c_{j}\left(g_{j+1}^{-1}, \ldots, g_{k}^{-1} g_{k+1}^{-1} \ldots, g_{p+1}^{-1}\right)\left(v_{\iota_{j}}\right) .
\end{aligned}
$$

On the other hand, we have that

$$
\begin{aligned}
\bar{\delta}\left(\lambda\left(c_{j}\right)\right)(v)= & (-1)^{l} \sum_{i=0}^{p+1}(-1)^{i} \lambda\left(c_{j}\right) d_{i}(v) \\
= & (-1)^{l}(-1)^{j} \sum_{i=j+1}^{p+1}(-1)^{i}\left(c_{j}\right)\left(g_{j+1}^{-1}, \ldots, g_{i}^{-1} g_{i+1}^{-1}, \ldots, g_{p+1}^{-1}\right)\left(v_{\iota_{j}}\right) \\
& +(-1)^{l}(-1)^{j}(-1)^{j} c_{j}\left(g_{j+2}^{-1}, \ldots, g_{p+1}^{-1}\right)\left(d_{j}(v)_{\iota_{j}}\right)+\ldots \\
& +(-1)^{l}(-1)^{j}(-1)^{1} c_{j}\left(g_{j+2}^{-1}, \ldots, g_{p+1}^{-1}\right)\left(d_{1}(v)_{\iota_{j}}\right) \\
& +(-1)^{l}(-1)^{j}(-1)^{0} c_{j}\left(g_{j+2}^{-1}, \ldots, g_{p+1}^{-1}\right)\left(d_{0}(v)_{\iota_{j}}\right) \\
= & (-1)^{l+j} \sum_{i=j+1}^{p+1}(-1)^{i}\left(c_{j}\right)\left(g_{j+1}^{-1}, \ldots, g_{i}^{-1} g_{i+1}^{-1}, \ldots, g_{p+1}^{-1}\right)\left(v_{\iota_{j}}\right) \\
& +(-1)^{l+j+j} c_{j}\left(g_{j+2}^{-1}, \ldots, g_{p+1}^{-1}\right)\left(v_{\delta_{j} \iota_{j}}\right)+\cdots \\
& +(-1)^{l+j+1} c_{j}\left(g_{j+2}^{-1}, \ldots, g_{p+1}^{-1}\right)\left((v)_{\delta_{1} \iota_{j}}\right) \\
& +(-1)^{l+j} c_{j}\left(g_{j+2}^{-1}, \ldots, g_{p+1}^{-1}\right)\left(\sum_{k=0}^{j}(-1)^{j} R_{j+1-k}^{\left(g_{j+1}, \ldots, g_{k+1}\right)} v_{\iota_{k}}\right) \\
& +(-1)^{l+j} c_{j}\left(g_{j+2}^{-1}, \ldots, g_{p+1}^{-1}\right)\left(\sum_{k=1}^{j}(-1)^{k+1} v_{\delta_{k} \iota_{j}}\right)
\end{aligned}
$$

So, in the computation of $\bar{\delta}\left(\left(\lambda\left(c_{j}\right)\right)\right)(v)$, all the terms of the form $c_{j}\left(g_{j+2}^{-1}, \ldots, g_{p+1}^{-1}\right)\left(v_{\delta_{k} \iota_{j}}\right)$ appear two times with opposite signs, thus cancelling each other. The remaining ones are exactly those appearing in $\lambda\left(D^{\prime}\left(c_{j}\right)\right)(v)$. Therefore, the diagram commutes and we have a map of cochain complexes. 
Remark 4.1.2. Even though we had to change the simplicial differential $\delta$ for $(-1)^{l} \delta$ to get a morphism of cochain complexes we remark that this does not cause any harm when we pass to cohomology, since the complexes $\left(C_{\operatorname{lin}}^{\bullet}(V), \delta\right)$ and $\left(C_{\text {lin }}^{\bullet}(V), \bar{\delta}\right)$ compute the same cohomology.

In what follows, we want to identify which cochains inside $C_{\text {lin }}^{\bullet}(V)$ are in the image of the map $\lambda$, or in other words which subcomplex of the linear complex is isomorphic to the complex of the dual representation up to homotopy we started with. By the definition of $\lambda$ and the previous proposition, it follows that for a cochain $\phi: V_{p} \rightarrow \mathbb{R}$ to be in the image of $\lambda$, a necessary condition is that $\phi$ (respectively $\delta(\phi))$ must depend only on the components $v_{\iota_{j}}$ indexed by inclusions $\iota_{j}:[j] \rightarrow[n]$ (respectively $\iota_{j}:[j] \rightarrow[n+1]$ ) of a vector $v \in V_{p}$ (respectively $v \in V_{p+1}$ ). Thus, we make the following definition:

Definition 4.1.3. Let $\mathrm{V}$ be the simplicial vector bundle coming from a representation up to homotopy. A cochain $\phi \in C_{\text {lin }}^{p}(V)$ is called projectable if it satisfies the following conditions:

- $\phi(v)=0$, for every $v \in V_{p}$ such that $v_{\iota_{k}}=0$, for every $\iota_{k}:[k] \rightarrow[p]$ with $0 \leqslant k \leqslant p$.

- $\delta(\phi)(w)=0$, for every $w \in V_{p+1}$ such that $w_{\iota_{k}}=0$, for every $\iota_{k}:[k] \rightarrow[p+1]$ with $0 \leqslant k \leqslant p+1$.

Then, we define the subcomplex $C_{\text {proj }}^{\bullet}(V) \subset C_{\text {lin }}^{\bullet}(V)$ consisting of all projectable cochains, and we denote its cohomology by $H_{\text {proj }}^{\bullet}(V)$.

Theorem 4.1.4. The image of the cochain map $\lambda:\left(C\left(\mathcal{G}, \mathcal{E}^{*}\right)^{\bullet-l}, D^{\prime}\right) \rightarrow\left(C_{\operatorname{lin}}^{\bullet}(V), \bar{\delta}\right)$ is exactly the subcomplex of projectable cochains. Thus, $\lambda$ gives rise to an isomorphism of complexes between $C\left(\mathcal{G}, \mathcal{E}^{*}\right)^{\bullet}$, and the complex of projectable cochains shifted byl, $C_{\text {proj }}^{\bullet}(V)[l]$. Therefore, it also induces an isomorphism in cohomology between $H^{\bullet}\left(\mathcal{G}, \mathcal{E}^{*}\right)$ and $H_{\text {proj }}^{\bullet}(V)[l]$.

Proof: From the definition of the complex of projectable cochains and Proposition 4.1.1, we have that the image of $\lambda$ is contained in $C_{\text {proj }}^{\bullet}(V)$. We only need to show that $\lambda$ is surjective. Let $\phi: V_{p} \rightarrow \mathbb{R}$ be any projectable cochain. We will show that its restriction to each summand $\pi_{j}^{*} E_{j} \subset V_{p}$, corresponding to the index map $i_{j}:[j] \rightarrow[p]$, can be identified with a section $c_{j} \in C^{p-j}\left(\mathcal{G}, E_{j}^{*}\right)$. For instance, in order to define $c_{0} \in C^{p}\left(\mathcal{G}, E_{0}^{*}\right)$, we use the canonical pairing $\langle$,$\rangle between E_{0}$ and its dual $E_{0}^{*}$, so that this section is uniquely determined by:

$$
\left\langle c_{0}\left(g_{1}^{-1}, \ldots, g_{p}^{-1}\right), \quad\right\rangle:=\left.\phi\right|_{\pi_{0}^{*} E_{0}}:\left.\pi_{0}^{*} E_{0}\right|_{\left(g_{p}, \ldots, g_{1}\right)} \rightarrow \mathbb{R} .
$$

As for the other sections, we proceed in a similar fashion using the pairing $\langle$,$\rangle between$ $E_{j}$ and its dual $E_{j}^{*}$ and defining

$$
\left\langle c_{j}\left(g_{j+1}^{-1}, \ldots, g_{p}^{-1}\right), \quad\right\rangle:=\left.(-1)^{j} \phi\right|_{\pi_{j}^{*} E_{j}}:\left.\pi_{j}^{*} E_{j}\right|_{\left(g_{p}, \ldots, g_{j+1}, 1, \ldots, 1\right)} \rightarrow \mathbb{R} .
$$


With these definitions, it follows that $\left(c_{0}, c_{1}, \ldots, c_{l}\right) \in C\left(\mathcal{G}, \mathcal{E}^{*}\right)^{p-l}$ is a well-defined element, and that $\lambda\left(c_{0}, c_{1}, \ldots, c_{l}\right)=\phi$ if, and only if, for each $j \in\{1, \ldots, l\}$ and for every $\left.e_{j} \in E_{j}\right|_{t\left(g_{j}\right)}$, the map $\left.\phi\right|_{\pi_{j}^{*} E_{j}}$ satisfies the identity

$$
\left(\left.\phi\right|_{\pi_{j}^{*} E_{j}}\right)\left(\left.e_{j}\right|_{\left(g_{p}, \ldots, g_{1}\right)}\right)=\left(\left.\phi\right|_{\pi_{j}^{*} E_{j}}\right)\left(\left.e_{j}\right|_{\left(g_{p}, \ldots, g_{j+1}, 1, \ldots, 1\right)}\right) .
$$

The fact that this identity holds true for any projectable cochain $\phi$ will be a consequence of Lemma 4.3.2. Thus, we can assume it holds, and the proof is finished.

Note that, by the definition of a simplicial vector bundle $V$ from a representation up to homotopy, we always have a natural projection $\pi_{q}: V_{p+q} \rightarrow V_{q}$, such that $\pi_{q}\left(v_{\alpha}\right)=v_{\beta}$ if, and only if, $\alpha=\iota \circ \beta:\{0, \ldots, k\} \rightarrow\{0, \ldots, p+q\}$, where $\iota:\{0, \ldots, q\} \rightarrow\{0, \ldots, p+q\}$ is the inclusion. This gives a canonical left $C^{\bullet}(\mathcal{G})$-module structure on the space $C_{\text {lin }}^{\bullet}(V)$ defined by

$$
C^{\bullet}(\mathcal{G}) \times C_{\operatorname{lin}}^{\bullet}(V) \rightarrow C_{\operatorname{lin}}^{\bullet}(V),
$$

where $(f, \phi) \in C^{p}(\mathcal{G}) \times C_{\text {lin }}^{q}(V)$ maps to $(f \phi): V_{p+q} \rightarrow \mathbb{R}$, given by

$$
(f \phi)(v):=f\left(g_{p+q}, \ldots, g_{q+1}\right) \phi\left(\pi_{q}(v)\right) .
$$

Using the inversion map of the Lie groupoid, we can transform any left module structure on a right module structure, for instance, we can define $\phi f:=f^{-1} \phi$ where $f^{-1}\left(g_{p}, \ldots, g_{1}\right):=$ $f\left(g_{1}^{-1}, \ldots, g_{p}^{-1}\right)$. Therefore, the linear complex $C_{\text {lin }}^{\bullet}(V)$ of a simplicial vector bundle $V$ coming from a representation up to homotopy can be seen as right-module over $C^{\bullet}(\mathcal{G})$, and we can prove the following:

Proposition 4.1.5. The map $\lambda:\left(C\left(\mathcal{G}, \mathcal{E}^{*}\right)^{\bullet-l}, D^{\prime}\right) \rightarrow\left(C_{\text {lin }}^{\bullet}(V), \delta\right)$ defined above is a morphism of right $C^{\bullet}(\mathcal{G})$-modules.

Proof: It follows from a straightforward computation.

\subsection{Morita invariance of the linear cohomology}

Our main goal is to prove the Morita invariance of the cohomology with coefficients in a representation up to homotopy. More precisely, suppose that $\phi: \mathcal{G} \rightarrow \mathcal{H}$ is a Morita fibration and that $\left(C(\mathcal{H}, \mathcal{E})^{\bullet}, D\right)$ is a representation up to homotopy of $\mathcal{H}$, then we have a pullback representation $\left(C\left(\mathcal{G}, \phi^{*} \mathcal{E}\right)^{\bullet}, \phi^{*} D\right)$ of $\mathcal{G}$, and we want to show that the cohomologies $\left(H_{\text {diff }}(\mathcal{H}, \mathcal{E})\right)^{\bullet}$ and $\left(H_{\text {diff }}\left(\mathcal{G}, \phi^{*} \mathcal{E}\right)\right)^{\bullet}$ are isomorphic.

We will start by showing, in this section, the Morita invariance of the linear cohomology of the simplicial vector bundles coming from a representation and its pullback. 
Theorem 4.2.1. Let $\phi: \mathcal{G} \rightarrow \mathcal{H}$ be a Morita fibration of Lie groupoids. Consider $W \rightarrow \mathcal{H}$. and $V \rightarrow \mathcal{G}_{\bullet}$, the simplicial vector bundles associated to a representation $\left(C(\mathcal{H}, \mathcal{E})^{\bullet}, D\right)$ and it its pullback $C\left(\mathcal{G}, \phi^{*} \mathcal{E}, \phi^{*} D\right)$ respectively. Then, $V$ is exactly the pullback simplicial vector bundle $V=\phi^{*} W$, and the pullback map $\Phi: V=\phi^{*} W \rightarrow W$ is a linear hypercover.

Proof: Assume $\mathcal{E}$ is a $(l+1)$-term complex $0 \rightarrow E_{l} \rightarrow \cdots \rightarrow E_{1} \rightarrow E_{0} \rightarrow 0$ so that $W$ is defined by

$$
W_{p}=\bigoplus_{[p] \stackrel{\alpha}{\rightarrow[k]}} \pi_{\alpha^{\prime}(k)}^{*} E_{k} .
$$

Then, the pullback representation $\phi^{*} \mathcal{E}$ is a representation of $\mathcal{G}$ on the pullback complex $0 \rightarrow \phi_{0}^{*} E_{l} \rightarrow \cdots \rightarrow \phi_{0}^{*} E_{1} \rightarrow \phi_{0}^{*} E_{0} \rightarrow 0$, and $V$ is defined by

$$
V_{p}=\bigoplus_{[p] \rightarrow[k]} \pi_{\alpha^{\prime}(k)}^{*} \phi_{0}^{*} E_{k}
$$

Since the extended map between the nerves, $\phi: \mathcal{G}_{\bullet} \rightarrow \mathcal{H}_{\bullet}$, is a map of simplicial manifolds it commutes with all vertex maps, i.e. for every $j \in\{0, \ldots, p\}$ the diagram below commutes

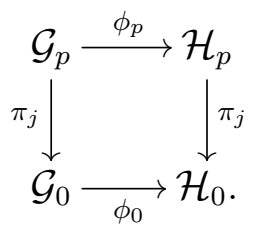

Therefore, we have that

$$
V_{p}=\bigoplus_{[p] \rightarrow[k]} \pi_{\alpha^{\prime}(k)}^{*} \phi_{0}^{*} E_{k}=\bigoplus_{[p] \rightarrow[k]} \phi_{p}^{*} \pi_{\alpha^{\prime}(k)}^{*} E_{k}=\phi_{p}^{*} W_{p}
$$

This proves that $V$ is the pullback of $W$. The last part of the statement follows from Lemma 2.3.7, because $\phi$ being a Morita fibration (a hypercover) implies that its pullback $\Phi: V=\phi^{*} W \rightarrow W$ must be a linear hypercover.

Corollary 4.2.2. Let $W$ and $V$ be the simplicial vector bundles associated to representations $\left(C(\mathcal{H}, \mathcal{E})^{\bullet}, D\right)$ and its pullback $C\left(\mathcal{G}, \phi^{*} \mathcal{E}, D^{\prime}\right)$ respectively, where $\phi: \mathcal{G} \rightarrow \mathcal{H}$ is Morita fibration. Then, there is a linear hypercover $\Phi: V \rightarrow W$ and consequently an isomorphism between linear cohomologies $\Phi^{*}: H_{\operatorname{lin}}^{\bullet}(W) \rightarrow H_{\operatorname{lin}}^{\bullet}(V)$

Proof: It follows from Theorem 4.2.1 that there is $\Phi: V \rightarrow W$ linear hypercover and from Corollary 3.2.3 we get an isomorphism in cohomology. 


\subsection{Morita invariance of the cohomology with values in a representation up to homotopy}

In this section, we will show that the cohomology with coefficients in a representation up to homotopy is a Morita invariant. In order to do that, we study the relationship between the cohomology of the complex of projectable cochains and the linear cohomology. We will see that as it is known to happen for two-term representations [12], in general, the inclusion map $C_{\text {proj }}^{\bullet}(V) \hookrightarrow C_{\text {lin }}^{\bullet}(V)$ is also a quasi-isomorphism. We are going to prove this result using a technique that was inspired by that of [12] and [19].

The idea is to define a filtration $F^{m}$ of the linear complex $C_{\operatorname{lin}}^{\bullet}(V)$ starting at the complex of projectable cochains $C_{\text {proj }}^{\bullet}(V)$ in such a way that each subcomplex in the filtration is quasi-isomorphic to the next one.

Let $V$ be the simplicial vector bundle coming from a representation up to homotopy of $\mathcal{G}$ on a graded vector bundle $E_{\text {. }}$. Then, each $V_{p}$ is a direct sum

$$
V_{p}=\bigoplus_{[k] \stackrel{\alpha}{\rightarrow}[p]} \pi_{\alpha(k)}^{*} E_{k}
$$

indexed by all order-preserving injective maps $\alpha:[k] \rightarrow[p]$, such that $\alpha(0)=0$, with $k \leqslant p$.

Remark 4.3.1. In what follows, for any index $\alpha:[k] \rightarrow[p], v_{\alpha}$ will denote corresponding component of a vector $v \in V_{p}$. If $\phi: V_{p} \rightarrow \mathbb{R}$ is a linear cochain, $\phi_{\alpha}$ will denote the restriction of $\phi$ to the summand $\pi_{\alpha(k)}^{*} E_{k}$. Moreover, if $v \in E_{k}$, then $v[\alpha] \in V_{p}$ will be the vector such that $(v[\alpha])_{\alpha}=v$ and $(v[\alpha])_{\beta}=0$, for every $\beta \neq \alpha$.

We define a sequence of subsets of $V$ in each degree $p$ in terms of the coordinates $\alpha$ : $[k] \rightarrow[p]$. For instance, $F_{0}(V)_{p}=\left\{v \in V_{p} \mid v_{\alpha}=0\right.$ for every $\alpha$ such that $\left.\alpha(0)=0\right\}$, $F_{1}(V)_{p}=\left\{v \in V_{p} \mid v_{\alpha}=0\right.$ for every $\alpha$ such that $\alpha(i)=i$ for every $\left.i \leqslant 1\right\}, F_{2}(V)_{p}=$ $\left\{v \in V_{p} \mid v_{\alpha}=0\right.$ for every $\alpha$ such that $\alpha(i)=i$ for every $\left.i \leqslant 2\right\}$. And in general, we define $F_{m}(V)_{p}=\left\{v \in V_{p} \mid v_{\alpha}=0\right.$ for every $\alpha$ such that $\alpha(i)=i$ for every $\left.i \in\{0,1,2, \ldots, m\}\right\}$.

Thus, for any degree $p$, we get an increasing chain of inclusions:

$$
0=F_{0}(V)_{p} \subset F_{1}(V)_{p} \subset \cdots \subset F_{p-1}(V)_{p}=F_{p}(V)_{p} .
$$

Now, we define $F^{m}(V)^{p}:=\left\{\phi \in C_{\text {lin }}^{p}(V) \mid \phi(v)=0\right.$, for every $\left.v \in F_{m}(V)_{p}\right\} \subset$ $C_{\text {lin }}^{p}(V)$, which is simply the annihilator of $F_{m}(V)_{p}$. Note that, any element $\phi \in F^{m}(V)$ is then characterized by the fact that $\phi_{\alpha}=0$ for any $\alpha$ such that $\alpha(m)>m$. In particular, any $\phi \in F^{p}(V)$ such that $\delta(\phi) \in F^{p}(V)^{p+1}$ is a projectable cochain as in Definition 4.1.3. Then, what we have is a dualized increasing filtration starting with $F^{p-1}(V)$ and ending at 
$C_{\text {lin }}(V):$

$$
F^{p}(V)^{p}=F^{p-1}(V)^{p} \subset F^{p-2}(V)^{p} \subset \cdots \subset F^{0}(V)^{p}=C_{\mathrm{lin}}^{p}(V)
$$

The idea now is to prove that any linear cocycle $\phi \in C_{\text {lin }}^{p}(V)$ is in the same cohomology class of some $\tilde{\phi} \in F^{p}(V)^{p}$, which will then be projectable, but going step by step i.e. showing that any cocycle $\phi \in F^{m}(V)^{p}$ can be exchanged for another one inside $F^{m+1}(V)^{p}$ in the same cohomology class.

We can also think of each $F^{m}(V)$ as a complex itself, modifying a little its definition. If we define, for instance, $\hat{F}^{m}(V)^{p}=\left\{\phi \in F^{m}(V)^{p} \mid \delta(\phi) \in F^{m}(V)^{p+1}\right\}$, then the restriction of the differential $\delta$ is also a differential turning $\left(\hat{F}^{k}(V)^{\bullet}, \delta\right)$ into a cochain complex.

Lemma 4.3.2. Consider $\phi \in \hat{F}^{m}(V)^{p}$, and an index $\alpha:[k] \rightarrow[p]$, satisfying $\alpha(i)=i$, for every $i \leqslant m \leqslant k$, and $v \in E_{k}$. Then, we have that $\phi\left(\left.v[\alpha]\right|_{\left(g_{p}, \ldots, g_{1}\right)}\right)=\phi\left(\left.v[\alpha]\right|_{\left(g_{p}, \ldots, g_{m+1}, 1,1, \ldots, 1\right)}\right)$. That is, $\phi(v[\alpha])$ does not depend on the first $m$ arrows of the string $\left(g_{p}, \ldots, g_{1}\right)$ that is the projection of $v[\alpha] \in V_{p}$ to the base $\mathcal{G}_{p}$.

Proof: Starting with $\phi, \alpha$ and $v$ as in the hypothesis, we can consider the vector $v\left[\delta_{m} \alpha\right] \in$ $V_{p+1}$ in the fibre over $\left(g_{p}, \ldots, g_{m+1}, 1, g_{m}, g_{m-1}, \ldots, g_{1}\right)$. Since $\delta_{m}(m)=m+1$ and $\delta(\phi) \in$ $F^{m}(V)^{p+1}$, it follows that $\delta(\phi)_{\left(\delta_{m} \alpha\right)}=0$. Consequently, we have

$$
0=\delta(\phi)\left(v\left[\delta_{m} \alpha\right]\right)=\sum_{j=0}^{p+1}(-1)^{j} \phi d_{j}\left(v\left[\delta_{m} \alpha\right]\right)
$$

Now we analyse each term in the sum above. If $j>m$, using the formula of the face map $d_{j}$, we have that $d_{j}\left(v\left[\delta_{m} \alpha\right]\right)_{\beta}= \pm v$ if, and only if, $\delta_{m} \alpha=\delta_{j} \beta$, which implies that $\beta(m)=\delta_{j} \beta(m)=\delta_{m} \alpha(m)=m+1$. Thus, $\phi\left(d_{j}\left(v\left[\delta_{m} \alpha\right]\right)\right)=0$ because $\phi \in F^{m}(V)^{p}$. If $m>j>0$, then $j \in \operatorname{Im}\left(\delta_{m} \alpha\right)$ implying that $d_{j}\left(v\left[\delta_{m} \alpha\right]=0\right.$ in this case. So, most of the terms in the sum above are zero and we are left with only two:

$$
0=\delta(\phi)\left(v\left[\delta_{m} \alpha\right]\right)=\sum_{j=0}^{p+1}(-1)^{j} \phi d_{j}\left(v\left[\delta_{m} \alpha\right]\right)=(-1)^{m} \phi\left(d_{m}\left(v\left[\delta_{m} \alpha\right]\right)\right)+\phi\left(d_{0}\left(v\left[\delta_{m} \alpha\right]\right)\right)
$$

Using the formulas for $d_{m}$ and $d_{0}$, and the hypothesis that $\phi \in F^{m}(V)^{p}$, it follows that

$$
(-1)^{m} \phi\left(d_{m}\left(v\left[\delta_{m} \alpha\right]\right)\right)=(-1)^{m} \phi(v[\alpha]) \text { and } \phi\left(d_{0}\left(v\left[\delta_{m} \alpha\right]\right)\right)=\phi\left((-1)^{m+1}(v[\alpha])\right) .
$$

Therefore, we get that

$$
(-1)^{m} \phi(v[\alpha])=(-1)^{m} \phi\left(d_{m}\left(v\left[\delta^{m} \alpha\right]\right)\right)=-\phi\left(d_{0}\left(v\left[\delta^{m} \alpha\right]\right)\right)=(-1)^{m} \phi((v[\alpha])),
$$

where the first term on the left is $\phi$ applied to the vector $v[\alpha] \in V_{p}$ sitting on the fibre over $\left(g_{p}, \ldots, g_{m+1}, g_{m}, g_{m-1}, \ldots, g_{1}\right)$, and the last term on the right is $\phi$ applied to the vector 
$v[\alpha] \in V_{p}$ on the fibre over $\left(g_{p}, \ldots, g_{m+1}, 1, g_{m}, g_{m-1}, \ldots, g_{2}\right)$. Thus, we can repeat the same argument above, putting one more unit at a time in the base, without changing the value of $\phi$, until we have replaced all the arrows $g_{i}$ with $1_{t\left(g_{m}\right)}$, for $0 \leqslant i \leqslant m$. This finishes the proof of the lemma.

Lemma 4.3.3. Every inclusion $\left(\hat{F}^{m+1}(V)^{\bullet}, \delta\right) \hookrightarrow\left(\hat{F}^{m}(V)^{\bullet}, \delta\right)$ is a quasi-isomorphism, for $0 \leqslant m \leqslant p-1$.

Proof: We are going to define a map which will play a role similar to the one played by a homotopy operator. First we define a map between bundles $h: V_{p} \rightarrow V_{p+1}$ by the formula:

$$
h(v)_{\beta}= \begin{cases}v_{\alpha} & \text { if } \beta=\delta_{m+1} \alpha \\ 0 & \text { otherwise. }\end{cases}
$$

Covering, in the base, the map also denoted by $h: \mathcal{G}_{p} \rightarrow \mathcal{G}_{p+1}$ :

$$
\left(g_{p}, \ldots, g_{1}\right) \stackrel{h}{\rightarrow}\left(g_{p}, \ldots, g_{m+1},\left(g_{p} g_{p-1} \cdots g_{m+2} g_{m+1}\right)^{-1},\left(g_{p} g_{p-1} \cdots g_{m}\right), g_{m-1}, \ldots, g_{1}\right)
$$

which we think of as doing a cone on the $m$-th vertex. Then, for any $p \geqslant 0$, we define the operator $I: C_{\text {lin }}^{p}(V) \rightarrow C_{\text {lin }}^{p}(V)$ by $I(\phi)=(-1)^{m} \phi+h^{*} \delta \phi+\delta h^{*} \phi$, which satisfies

$$
\begin{aligned}
\delta(I(\phi)) & =\delta\left((-1)^{m} \phi+h^{*} \delta \phi+\delta h^{*} \phi\right) \\
& =(-1)^{m} \delta \phi+\delta h^{*} \delta \phi+\delta^{2} h^{*} \phi \\
& =(-1)^{m} \delta \phi+\delta h^{*} \delta \phi \\
& =I(\delta \phi) .
\end{aligned}
$$

Thus, $I$ is a morphism of complexes and we want to show that taking any $\phi \in \hat{F}^{m}(V)$ and applying $I$ to it a number of times we will have in the end an element of $\hat{F}^{m+1}(V)$. This will show that some power of $I$ is an inverse of the inclusion $\hat{F}^{m+1}(V) \hookrightarrow \hat{F}^{m}(V)$ in cohomology.

Suppose that $\phi \in F^{m}(V)^{p}$ and that it is not in $F^{m+1}(V)^{p}$. Then, there is some index $\alpha:[k] \rightarrow[p]$ such that $\alpha(i)=i, \forall i \leqslant m, \alpha(m+1)>m+1$, and $\phi_{\alpha} \neq 0$. Let $n_{0}$ be the number

$$
n_{0}=\max \left\{\alpha(m+1) \mid \alpha(i)=i, \forall i \leqslant m, \text { and } \phi_{\alpha} \neq 0\right\} .
$$

We will show now that $\max \left\{\alpha(m+1) \mid \alpha(i)=i, \forall i \leqslant m\right.$, and $\left.I(\phi)_{\alpha} \neq 0\right\} \leqslant n_{0}-1$. Consider any $\alpha:[k] \rightarrow[p]$, such that $\alpha(m)=m, \alpha(m+1) \geqslant n_{0}$ and $v \in E_{k}$. Using the notation $v[\alpha]$ for the element of $V_{p}$, that has $v$ in the coordinate $\alpha$ and zero elsewhere, we want to prove that $I(\phi)(v[\alpha])=0$, which is equivalent to proving that $I(\phi)_{\alpha}=0$. Doing 
the computation we get:

$$
\begin{aligned}
I(\phi)(v[\alpha]) & =\left((-1)^{m} \phi+h^{*} \delta \phi+\delta h^{*} \phi\right)(v[\alpha]) \\
& =(-1)^{m} \phi(v[\alpha])+\delta(\phi)(h v[\alpha])+\delta(\phi \circ h)(v[\alpha]) \\
& =(-1)^{m} \phi(v[\alpha])+\sum_{j=0}^{p+1}(-1)^{j} d_{j}^{*} \phi(h v[\alpha])+\sum_{i=0}^{p}(-1)^{i} d_{i}^{*}(\phi \circ h)(v[\alpha]) \\
& =(-1)^{m} \phi(v[\alpha])+\sum_{j=0}^{p+1}(-1)^{j} \phi\left(d_{j} h v[\alpha]\right)+\sum_{i=0}^{p}(-1)^{i}(\phi \circ h)\left(d_{i} v[\alpha]\right) .
\end{aligned}
$$

Thus, in order to show that $I(\phi)(v[\alpha])=0$, we will prove that $\phi\left(d_{j+1} h v[\alpha]\right)=\phi\left(h d_{j} v[\alpha]\right)$, for any $j \geqslant m+1, \phi\left(d_{j} h(v[\alpha])\right)=0=\phi\left(h d_{j}(v[\alpha])\right)$, for every $j \in\{1, \ldots, m-1\}$, and that $\phi\left(d_{0}(h v[\alpha])\right)=(-1)^{m+1} \phi(v[\alpha]), \phi\left(d_{m+1}(h v[\alpha])\right)=-\phi\left(h d_{0}(v[\alpha])\right)$. So that, all the terms appearing in the computation above cancel in pairs.

We have some cases to consider:

Case A: If $j \geqslant \alpha(m+1), j+1 \geqslant \alpha(m+1)+1=\left(\delta_{m+1} \alpha\right)(m+1) \geqslant n_{0}+1$. In this case, $d_{j+1} h(v[\alpha])=d_{j+1}\left(v\left[\delta_{m+1} \alpha\right]\right)$, and by the formula for $d_{j+1}$, we know that $\left(d_{j+1}\right)_{\left(\delta_{m+1} \alpha\right) \beta} \neq 0$ if, and only if, $\delta_{m+1} \alpha=\beta \delta_{j+1}$. But then, $\beta \delta_{j+1}(m+1) \geqslant n_{0}+1$ implies that $\beta(m+1) \geqslant n_{0}+1$, and by our hypothesis $\phi_{\beta}=0$ for any such $\beta$. Therefore, we have that $\phi\left(d_{j+1}(h v[\alpha])\right)=0$.

On the other hand, we have $h d_{j}(v[\alpha])$. By the same reasoning as above, $d_{j}(v[\alpha])_{\beta}$ can be non-zero only if, $\alpha=\beta \delta_{j}$, implying that $\beta(m+1) \geqslant \alpha(m+1) \geqslant n_{0}$. Since $h(v[\beta])=v\left[\delta_{m+1} \beta\right]$, we have that $h\left(d_{j}(v[\alpha])\right)_{\gamma}$ may be non-zero only if, $\delta_{m+1} \beta=\gamma$. But then again, $\gamma(m+1)=\delta_{m+1} \beta(m+1) \geqslant n_{0}+1, \phi_{\gamma}=0$ for any such $\gamma$, and we get $\phi\left(h d_{j}(v[\alpha])\right)=0$.

Case B: If $\alpha(m+1)>j \geqslant m+1 ; \alpha(m+1)+1>j+1 \geqslant m+2$. Then, $d_{j+1} h(v[\alpha])=$ $d_{j+1}\left(v\left[\delta_{m+1} \alpha\right]\right)$, and $d_{j+1}\left(v\left[\delta_{m+1} \alpha\right]\right)_{\beta}=v$ if, and only if, $\delta_{m+1} \alpha=\delta_{j+1} \beta$. Applying a codegeneracy map, we can write this in an equivalent way as $d_{j+1}\left(v\left[\delta_{m+1} \alpha\right]\right)_{\beta}=v$ if, and only if, $\sigma_{j+1} \delta_{m+1} \alpha=\sigma_{j+1} \delta_{j+1} \beta=\beta$.

As for $\left(h d_{j}(v[\alpha])\right)_{\beta}$, first notice that $d_{j}(v[\alpha])_{\gamma}=v$ if, and only if, $\alpha=\delta_{j} \gamma$ if, and only if, $\gamma=\sigma_{j} \delta_{j} \gamma=\sigma_{j} \alpha$. Thus, $\left(h d_{j}(v[\alpha])\right)_{\beta}=\left(h\left(v\left[\sigma_{j} \alpha\right]\right)\right)_{\beta}=v$ if, and only if, $\beta=\delta_{m+1}\left(\sigma_{j} \alpha\right)$, and by cosimplicial identities, we know that $\delta_{m+1} \sigma_{j}=\sigma_{j+1} \delta_{m+1}$. This proves that $d_{j+1} h(v[\alpha])=h d_{j}(v[\alpha])$, as soon as, we show that they are vectors in the same fibre.

In the first case, $d_{j+1} h$ covers the map

$$
\begin{aligned}
& \left(g_{p}, \ldots, g_{1}\right) \stackrel{h}{\rightarrow}\left(g_{p}, \ldots, g_{m+1}, g_{m+1}^{-1} g_{m+2}^{-1} \cdots g_{p}^{-1},\left(g_{p} g_{p-1} \cdots g_{m}\right), g_{m-1}, \ldots, g_{1}\right) \rightarrow \\
& \stackrel{d_{j+1}}{\longrightarrow}\left(g_{p}, \ldots, g_{j+1} g_{j}, \ldots, g_{m+1}, g_{m+1}^{-1} g_{m+2}^{-1} \cdots g_{p}^{-1},\left(g_{p} g_{p-1} \cdots g_{m}\right), g_{m-1}, \ldots, g_{1}\right)
\end{aligned}
$$


In the second, $h d_{j}$ covers the map

$$
\begin{aligned}
& \left(g_{p}, \ldots, g_{1}\right) \stackrel{d_{j}}{\rightarrow}\left(g_{p}, \ldots, g_{j+1} g_{j}, \ldots, g_{1}\right) \rightarrow \\
& \stackrel{h}{\rightarrow}\left(g_{p}, \ldots, g_{j+1} g_{j}, \ldots g_{m+1}, g_{m+1}^{-1} g_{m+2}^{-1} \cdots g_{p}^{-1},\left(g_{p} g_{p-1} \cdots g_{m}\right), g_{m-1}, \ldots, g_{1}\right)
\end{aligned}
$$

This finishes the verification of case B.

Case C: This case consists of only the term $j=m ; j+1=m+1$. Then, $d_{m+1}(h(v[\alpha]))_{\beta}=$ $d_{m+1}\left(v\left[\delta_{m+1} \alpha\right]\right)_{\beta}=v$ if, and only if, $\delta_{m+1} \alpha=\delta_{m+1} \beta$. In other words, $d_{m+1} h([v[\alpha]])=$ $v[\alpha]$ in the fibre over $\left(g_{p}, \ldots, g_{m+2},\left(g_{m+2}^{-1} \cdots g_{p}^{-1}\right),\left(g_{p} g_{p-1} \cdots g_{m}\right), g_{m-1}, \ldots, g_{1}\right)$

Case D: Consider $j$, such that $m>j>0 ; m+1>j+1>1$. Then, $\phi\left(d_{j+1} h v[\alpha]\right)=$ $\phi\left(d_{j+1} v\left[\delta_{m+1} \alpha\right]\right)=0=\phi\left(h d_{j}(v[\alpha])\right)$, since, for any such $j$, we have that $j+1 \in$ $\operatorname{Im}\left(\delta_{m+1} \alpha\right)$ and $j \in \operatorname{Im}(\alpha)$.

Case E: This case consists of computing $\phi\left(d_{0}(h v[\alpha])\right)$, and $\phi\left(h d_{0}(v[\alpha])\right)$. Remember that, in the matrix $\left(d_{0}\right)_{\alpha \beta}$, the non-zero entries may be $\pm \operatorname{Id}$ or $\pm R_{m}$ for some $m$. The former occurs when $\operatorname{Im}(\alpha)=\operatorname{Im}(\bar{\beta}) \backslash\{\bar{\beta}(k)\}$, in which case $\left(d_{0}(v[\alpha])_{\beta}=(-1)^{k-1} \operatorname{Id}(v)\right.$. The latter occurs when $\operatorname{Im}(\alpha)=\operatorname{Im}(\bar{\beta}) \backslash\{\bar{\beta}(k+1), \ldots, \bar{\beta}(l+1)\}$, and we have that $\left(d_{0}\right)_{\alpha \beta}=(-1)^{l} R_{l-k+1}$.

Hence, $\left(d_{0} h(v[\alpha])\right)_{\beta}=\left(d_{0}\left(v\left[\delta_{m+1} \alpha\right]\right)\right)_{\beta}$ will be non-zero if $\operatorname{Im}\left(\delta_{m+1} \alpha\right)=\operatorname{Im}(\bar{\beta}) \backslash$ $\{\bar{\beta}(j)\}$ for some $j$, or if $\operatorname{Im}\left(\delta_{m+1} \alpha\right)=\operatorname{Im}(\bar{\beta}) \backslash\{\bar{\beta}(j+1), \ldots \bar{\beta}(l+1)\}$. In the former case, the only component that can contribute to $\phi\left(\left(d_{0} h(v[\alpha])\right)\right)$ is $\left(d_{0} h(v[\alpha])\right)_{\alpha}$, where $\operatorname{Im}\left(\delta_{m+1} \alpha\right)=\operatorname{Im}(\bar{\alpha}) \backslash\{\bar{\alpha}(m)\}$, since any other possible choice of $\beta$ will satisfy $\beta(m)>m$ and then, the component $\phi_{\beta}$ is zero. Moreover, in all the cases where $\left(d_{0}\left(v\left[\delta_{m+1} \alpha\right]\right)_{\beta}= \pm R\right.$, we have that $\beta(m+1) \geqslant n_{0}+1$, so $\phi_{\beta}=0$ by hypothesis, and these terms also do not contribute for $\phi\left(d_{0}(h v[\alpha])\right)$. Therefore, we conclude that $\phi\left(d_{0}(h v[\alpha])\right)=(-1)^{m+1} \phi(v[\alpha])$, where the $v[\alpha] \in V_{p}$ appearing on the right-hand side is in the fibre over $\left(g_{p}, \ldots, g_{m+1},\left(g_{m+1}^{-1} \cdots g_{p}^{-1}\right),\left(g_{p} \cdots g_{m}\right), g_{m-1}, \ldots, g_{2}\right)$.

With the same kind of reasoning applied to the computation $\phi\left(h d_{0}(v[\alpha])\right)$, one can show that, the only component of the vector $h d_{0}(v[\alpha])$, where $\phi$ does not vanish is $\alpha$ itself. Thus, we obtain $\phi\left(h d_{0}(v[\alpha])\right)=(-1)^{m} \phi(v[\alpha])$, where the $v[\alpha] \in V_{p}$ appearing on the right is in the fibre over

$$
\left(g_{p}, \ldots, g_{m+2},\left(g_{m+2}^{-1} \cdots g_{p}^{-1}\right),\left(g_{p} \cdots g_{m+1}\right), g_{m}, \ldots, g_{2}\right)
$$

After analysing all these cases, we see that most of the terms in the computation of $I(\phi)(v[\alpha])$ 
cancel out, and there are only four remaining:

$$
\begin{aligned}
I(\phi)(v[\alpha])= & (-1)^{m} \phi(v[\alpha])+(-1)^{0} \phi\left(d_{0} h v[\alpha]\right) \\
& +(-1)^{m+1} \phi\left(d_{m+1} h v[\alpha]\right)+(-1)^{0}(\phi \circ h)\left(d_{0} v[\alpha]\right) \\
= & (-1)^{m} \phi(v[\alpha])+(-1)^{m+1} \phi(v[\alpha])+(-1)^{m+1} \phi(v[\alpha])+(-1)^{m}(\phi)(v[\alpha]),
\end{aligned}
$$

where both the first pair and the last pair are over strings of $p$ composable arrows only differing by the first $m$ arrows. Thus, applying Lemma 4.3 .2 we get that these pairs also cancel in the computation above. This finishes the proof that $I(\phi)_{\alpha}=0$, for every $\alpha$ such that $\alpha(m)=m$ and $\alpha(m+1) \geqslant n_{0}$.

We conclude that each time we apply the map $I$ to a cochain $\phi \in \hat{F}^{m}(V)$, we have $\max \left\{\alpha(m+1) \mid \alpha(i)=i, \forall i \leqslant m\right.$, and $\left.I(\phi)_{\alpha} \neq 0\right\} \leqslant n_{0}-1<n_{0}=\max \{\alpha(m+1) \mid \alpha(i)=$ $i, \forall i \leqslant m$, and $\left.\phi_{\alpha} \neq 0\right\}$. Therefore, if we repeat this process at most $n_{0}-(m+1)$ times we will get that $I^{n_{0}-(m+1)}(\phi) \in F^{m+1}(V)^{p}$. Since $I$ commutes with $\delta$, a similar argument can be made for $I^{n_{1}-(m+1)}(\delta(\phi))=\delta\left(I^{n_{1}-(m+1)}(\phi)\right)$, with $n_{1}=\max \{\alpha(m+1) \mid \alpha(i)=i, \forall i \leqslant$ $m$, and $\left.(\delta(\phi))_{\alpha} \neq 0\right\}$. Thus, taking $\tilde{n}=\max \left\{n_{0}-(m+1), n_{1}-(m+1)\right\}$ we see that $I^{\tilde{n}}(\phi) \in \hat{F}^{m+1}(V)^{p}$.

The following theorem is a direct consequence.

Theorem 4.3.4. Suppose $V \rightarrow \mathcal{G}$ is a simplicial vector bundle coming from a representation up to homotopy of $\mathcal{G}$. Then, the inclusion $C_{\text {proj }}^{\bullet}(V) \hookrightarrow C_{\mathrm{lin}}^{\bullet}(V)$ is a quasi-isomorphism.

Proof: We know from Lemma 4.3.3 that each inclusion $\hat{F}^{m+1}(V) \hookrightarrow \hat{F}^{m}(V)$ is a quasiisomorphism, for $0 \leqslant m \leqslant p-1$. Moreover, for each $p \geqslant 0$ we have that $\hat{F}^{0}(V)^{p}=C_{\text {lin }}^{p}(V)$ and $\hat{F}^{p}(V)^{p}=C_{\text {proj }}^{p}(V)$. Therefore, we conclude that $C_{\text {proj }}^{\bullet}(V) \hookrightarrow C_{\text {lin }}^{\bullet}(V)$ is a quasiisomorphism.

Corollary 4.3.5. The differentiable cohomology of a Lie groupoid $\mathcal{G}$ with values in a representation up to homotopy is a Morita invariant.

Proof: Consider a Morita fibration $\phi: \mathcal{G} \rightarrow \mathcal{H}$, a representation of $\mathcal{H}$ on $\mathcal{E}$, and the pullback representation of $\mathcal{G}$ on $\phi^{*} \mathcal{E}$. Let $W \rightarrow \mathcal{H}$ and $V \rightarrow \mathcal{G}$ be the simplicial vector bundles associated to these representations of $\mathcal{H}$ and $\mathcal{G}$, respectively.

Then, by Theorem 4.1.4 we have isomorphisms in cohomology

$$
H_{\text {diff }}^{\bullet}\left(\mathcal{G}, \phi^{*} \mathcal{E}^{*}\right) \cong H_{\text {proj }}^{\bullet}(V)[l], \quad H_{\text {diff }}^{\bullet}\left(\mathcal{H}, \mathcal{E}^{*}\right) \cong H_{\text {proj }}^{\bullet}(W)[l]
$$

And by Theorem 4.3.4 and Corollary 4.2.2, we have isomorphisms

$$
H_{\text {proj }}^{\bullet}(W) \cong H_{\text {lin }}^{\bullet}(W) \cong H_{\text {lin }}^{\bullet}(V) \cong H_{\text {proj }}^{\bullet}(V)
$$

Therefore, $H_{\text {diff }}^{\bullet}\left(\mathcal{G}, \phi^{*} \mathcal{E}^{*}\right) \cong H_{\text {diff }}^{\bullet}\left(\mathcal{H}, \mathcal{E}^{*}\right)$. 


\section{Bibliography}

[1] C. A. Abad, M. Crainic, and B. Dherin. Tensor products of representations up to homotopy. Journal of Homotopy and Related Structures, 6(2):239-288, 2011.

[2] C. A. Abad and F. Schätz. Deformations of lie brackets and representations up to homotopy. Indagationes Mathematicae, 22(1):27-54, 2011.

[3] C. Arias Abad and M. Crainic. Representations up to homotopy and Bott's spectral sequence for Lie groupoids. Advances in Mathematics, 248:416-452, 2013.

[4] C. Arias Abad and F. Schätz. The $A_{\infty}$ de Rham theorem and integration of representations up to homotopy. International Mathematics Research Notices, Volume 2013(16):3790-3855, 2013.

[5] K. Behrend and E. Getzler. Geometric higher groupoids and categories. ArXiv e-prints, pages 1-40, 2015.

[6] K. Behrend and P. Xu. $S^{1}$-bundles and gerbes over differentiable stacks. Comptes Rendus Mathematique, 336(2):163-168, 2003.

[7] K. Behrend and P. Xu. Differentiable stacks and gerbes. F. Symplectic Geom., 9(3):285$341,2011$.

[8] K. A. Behrend. On the de Rham cohomology of differential and algebraic stacks. Advances in Mathematics, 198(2):583-622, 2005.

[9] C. Blohmann. Stacky Lie groups. International Mathematics Research Notices, 2008.

[10] O. Brahic and C. Ortiz. Integration of 2-term representations up to homotopy via 2-functors. Transactions of the American Mathematical Society, 372(1):503-543, 2019.

[11] H. Bursztyn, A. Cabrera, and M. del Hoyo. Vector bundles over Lie groupoids and algebroids. Advances in Mathematics, 290:163-207, 2016.

[12] A. Cabrera and T. Drummond. Van Est isomorphism for homogeneous cochains. Pacific fournal of Mathematics, 287(2):297-336, 2017. 
[13] A. Connes. Noncommutative Geometry. Elsevier Science, 1995.

[14] M. Crainic. Differentiable and algebroid cohomology, Van Est isomorphisms, and characteristic classes. Commentarii Mathematici Helvetici, 78(4):681-721, 2003.

[15] M. Crainic and R. Fernandes. Integrability of Lie brackets. Annals of Mathematics, 157(2):575-620, 2003.

[16] M. Crainic and J. N. Mestre. Orbispaces as differentiable stratified spaces. Letters in Mathematical Physics, 108(3):805-859, 2018.

[17] M. Crainic, J. N. Mestre, and I. Struchiner. Deformations of Lie Groupoids. International Mathematics Research Notices, 2018.

[18] M. Crainic and I. Struchiner. On the linearization theorem for proper lie groupoids. Annales scientifiques de l'École Normale Supérieure, Ser. 4, 46(5):723-746, 2013.

[19] M. del Hoyo and C. Ortiz. Morita Equivalences of Vector Bundles. International Mathematics Research Notices, rny149, 2018.

[20] M. Del Hoyo and D. Stefani. The general linear 2-groupoid. Pacific fournal of Mathematics, 298(1):33-57, 2019.

[21] M. del Hoyo and G. Trentinaglia. Simplicial vector bundles over lie groupoids. Work in progress, 2019.

[22] M. L. del Hoyo. Lie groupoids and their orbispaces. Portugaliae Mathematica, 70(2):161-209, 2013.

[23] J. J. Duistermaat and J. A. C. Kolk. Lie Groups. Universitext. Springer Berlin Heidelberg, 2012.

[24] P. Gabriel and M. Zisman. Calculus of fractions and homotopy theory. Ergebnisse der Mathematik und ihrer Grenzgebiete. Springer-Verlag, 1967.

[25] E. Getzler. Lie theory for nilpotent $L_{\infty}$-algebras. Annals of Mathematics, 170(1):271$301,2009$.

[26] E. Getzler. Differential forms on stacks. Slides from minicourse at Winter School in Mathematical Physics, Les Diablerets. 2014.

[27] P. Goerss and J. Jardine. Simplicial Homotopy Theory. Progress in mathematics (Boston, Mass.) v. 174. Springer, 1999.

[28] A. Gracia-Saz and R. A. Mehta. VB-groupoids and representation theory of lie groupoids. Journal of Symplectic Geometry, 15(3):741-783, 2017. 
[29] A. Haefliger. Groupoides d'holonomie et classifiants. In Structure transverse des feuilletages, number 116, pp. 70-97 in Astérisque. 1984.

[30] A. Henriques. Integrating $L_{\infty}$-algebras. Compositio Mathematica, 144(04):1017-1045, 2008.

[31] A. Joyal. Quasi-categories and kan complexes. Fournal of Pure and Applied Algebra, 175(1-3):207 - 222, 2002.

[32] N. P. Landsman. Lie groupoids and Lie algebroids in physics and noncommutative geometry, 2006.

[33] E. Lerman. Orbifolds as stacks?. L’Enseignement Mathématique (2), 56(3-4):315-363, 2010.

[34] E. Lerman and A. Malkin. Hamiltonian group actions on symplectic Deligne-Mumford stacks and toric orbifolds. Advances in Mathematics, 229(2):984-1000, 2012.

[35] D. Li-Bland and P. Severa. Integration of exact courant algebroids. Electronic Research Announcements in Mathematical Sciences, 19:58-76, 2012.

[36] J. Lurie. Higher Topos Theory (AM-170). Princeton University Press, 2009.

[37] K. Mackenzie. Lie Groupoids and Lie Algebroids in Differential Geometry. Lecture note series / London mathematical society. Cambridge University Press, 1987.

[38] K. C. H. Mackenzie. General Theory of Lie Groupoids and Lie Algebroids. London Mathematical Society Lecture Note Series. Cambridge University Press, 2005.

[39] J. May. Simplicial Objects in Algebraic Topology. Chicago Lectures in Mathematics. University of Chicago Press, 1992.

[40] R. A. Mehta and X. Tang. From double Lie groupoids to local Lie 2-groupoids. Bulletin of the Brazilian Mathematical Society, 42(4):651-681, 2011.

[41] R. A. Mehta and X. Tang. Constant symplectic 2-groupoids. Letters in Mathematical Physics, 108(5):1203-1223, 2018.

[42] R. A. Mehta and X. Tang. Symplectic structures on the integration of exact Courant algebroids. Journal of Geometry and Physics, 127:68-83, 2018.

[43] I. Moerdijk and J. Mrcun. Introduction to Foliations and Lie Groupoids. Cambridge Studies in Advanced Mathematics. Cambridge University Press, 2003.

[44] I. Moerdijk and D. A. Pronk. Orbifolds, Sheaves and Groupoids. K-Theory, 12(1):3-21, 1997. 
[45] J. P. Pridham. Presenting higher stacks as simplicial schemes. Advances in Mathematics, 238:184-245, 2013.

[46] G. Segal. Classifying spaces and spectral sequences. Publications Mathématiques de l'Institut des Hautes Scientifiques, 34(1):105-112, 1968.

[47] K. Wang. Proper Lie groupoids and their orbit spaces. Korteweg-de Vries Institute for Mathematics (KdVI), 2018.

[48] A. Weinstein. Symplectic groupoids and Poisson manifolds. Bull. Amer. Math. Soc. (N.S.), 16(1):101-104, 1987.

[49] J. Wolfson. Descent for n-bundles. Advances in Mathematics, 288:527-575, 2016.

[50] C. Zhu. N-groupoids and stacky groupoids. International Mathematics Research Notices, 2009(21):4087-4141, 2009. 